\title{
The universal Kobayashi-Hitchin correspondence on Hermitian manifolds
}

\author{
M. Lübke, A. Teleman
}

June 26, 2019

\section{Contents}

$\begin{array}{lll}0 & \text { Introduction } & 3\end{array}$

1 The finite dimensional Kobayashi-Hitchin correspondence 10

1.1 Analytic Stability, Symplectic stability . . . . . . . . . . . 10

1.2 The Continuity Method in the finite dimensional case . . . . . 16

1.3 Maximal weight functions for linear and projective actions . . . 20

1.3.1 Linear actions . . . . . . . . . . . . . . . . . 20

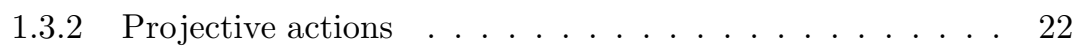

2 A "universal" complex geometric classification problem 23

2.1 Oriented holomorphic pairs . . . . . . . . . . . . . . 23

2.2 The stability condition for universal oriented holomorphic pairs . 25

2.2.1 The degree of a meromorphic $L$-reduction with respect to an ad-invariant linear form . . . . . . . . . . . 25

2.2.2 Stability, Semistability, Polystability . . . . . . . . . . 32

3 Hermitian-Einstein pairs $\quad 35$

3.1 The Hermitian-Einstein equation . . . . . . . . . . . . 35

3.2 Pairs which allow Hermitian-Einstein reductions are polystable . 37 
4.1 The perturbed equation $\ldots \ldots \ldots \ldots$. . . . . . . . 41

4.2 A priori estimates for the solution $s_{\varepsilon} \ldots \ldots \ldots \ldots 4$

4.3 Solving the equation $\left(e_{\varepsilon}\right)$ for $\varepsilon \in(0,1] \ldots \ldots \ldots \ldots$

4.4 Destabilizing the pair in the unbounded case . . . . . . . . 53

4.4.1 Estimates in the unbounded case . . . . . . . . . . 53

4.4.2 The properties of the limit $\sigma \ldots \ldots \ldots \ldots 5$

4.4.3 The limit $\sigma$ destabilizes $\ldots \ldots \ldots \ldots \ldots$

5 Appendix $\quad 64$

5.1 Chern connections . . . . . . . . . . . . . . . . 64

5.2 The orbits of the adjoint action. Sections in the adjoint bundle . 68

5.3 Local maximal torus reductions of a $K$-bundle $\ldots \ldots \ldots$. . . . 74

5.4 Decomposing a connection with respect to a maximal torus reduction. Applications . . . . . . . . . . . . 75

5.5 Analytic results . . . . . . . . . . . . . . . . . . . 79

5.6 Meromorphic parabolic reductions defined by weakly holomorphic $L_{1}^{2}$-sections . . . . . . . . . . . . . . 81

\begin{abstract}
We prove a very general Kobayashi-Hitchin correspondence on arbitrary compact Hermitian manifolds. This correspondence refers to moduli spaces of "universal holomorphic oriented pairs". Most of the classical moduli problems in complex geometry (e. g. holomorphic bundles with reductive structure groups, holomorphic pairs, holomorphic Higgs pairs, Witten triples, arbitrary quiver moduli problems) are special cases of this universal classification problem. Our Kobayashi-Hitchin correspondence relates the complex geometric concept "polystable oriented holomorphic pair" to the existence of a reduction solving a generalized HermitianEinstein equation. The proof is based on the Uhlenbeck-Yau continuity method.
\end{abstract}

MSC classification : 53C07, 32G13, 58D27, 53C55, 53D20, 32L05, 32M05 


\section{Introduction}

In order to understand the aim and the motivation of this article let us consider the following classical complex geometric moduli problems:

Holomorphic structures with fixed determinant. Let $X$ be a compact complex $n$-dimensional manifold and $E$ a differentiable rank $r$ vector bundle on $X$. We fix a holomorphic structure $\mathcal{L}$ on the determinant line bundle $\operatorname{det} E$ of $X$. The problem is to classify all holomorphic structures $\mathcal{E}$ on $E$ which induce the fixed holomorphic structure $\mathcal{L}$ on $\operatorname{det} E$ modulo the group $\Gamma(X, S L(E))$ of automorphisms of determinant 1 .

In order to get a Hausdorff moduli spaces with good properties one considers only stable (or more general semistable) holomorphic structures. The stability condition depends on the choice of a Gauduchon metric on $X$, or more generally a Hermitian metric $g$ (see $[\mathrm{LT}]$ ) which plays the same role as the choice of a polarization of the base manifold in algebraic geometry. $\mathcal{E}$ is called $g$-stable if

$$
\mu_{g}(\mathcal{F})<\mu_{g}(\mathcal{E})
$$

for any nontrivial subsheaf $\mathcal{F} \subset \mathcal{E}$ with torsion free quotient. Such a subsheaf must be reflexive. Note that the slope map $\mu_{g}$ is not a topological invariant in the general non-Kählerian framework, but it is always a holomorphic invariant.

The classical Kobayashi-Hitchin correspondence ([Ko1], [Do1], [Do2], [UY1], [UY2], [Bu], [LY], [LT]) states that a holomorphic structure is stable if and only if it is simple (i. e. it admits no non-trivial trace free infinitesimal automorphisms) and admits a Hermitian-Einstein metric, i. e. a metric $h$ solving the Hermitian-Einstein equation:

$$
i \Lambda_{g} F_{h}=\frac{2 \pi}{(n-1) ! V o l_{g}(X)} \mu_{g}(\mathcal{E}) \operatorname{id}_{E} .
$$

General solutions of the Hermitian-Einstein equation correspond to polystable holomorphic structures, i. e. to bundles which are direct sum of stable bundles of the same slope.

Let us fix a Hermitian metric $h$ on $E$. The Kobayashi-Hitchin correspondence yields an isomorphism of moduli spaces between the moduli space of stable holomorphic structures with fixed determinant and the moduli space of irreducible integrable Hermitian-Einstein connections $A$ with fixed determinant on $(E, h)$. This isomorphism plays a fundamental role in Donaldson theory, since - on complex surfaces - the latter moduli space can be further identified with a moduli space of $P U(r)$-instantons (see [DK], [LT]). 
Higgs pairs. We fix a differentiable vector bundle $E$ on $X$, a holomorphic structure $\mathcal{L}$ on $\operatorname{det} E$ and a holomorphic vector bundle $\mathcal{F}_{0}$ on $X$. In the classical theory of Higgs fields one takes $\mathcal{F}_{0}=\Omega_{X}^{1}$ (see [Hi], [Si]).

The problem here is to classify modulo $\Gamma(X, S L(E))$ all pairs $(\mathcal{E}, \Phi)$ where $\mathcal{E}$ is again a holomorphic structure on $E$ which induces $\mathcal{L}$ on $\operatorname{det} E$, and $\Phi \in$ $H^{0}\left(\operatorname{End}(\mathcal{E}) \otimes \mathcal{F}_{0}\right)$ is a holomorphic $\mathcal{F}_{0}$-twisted endomorphism.

For such objects one also has a stability condition: this condition asks that (1) holds for all nontrivial $\Phi$-invariant subsheaves $\mathcal{F}$. This complex geometric condition can be again characterized in a differential geometric way (see [Hi], $[\mathrm{Si}])$ : a Higgs holomorphic pair $(\mathcal{E}, \Phi)$ is stable if and only if is simple and $\mathcal{E}$ admits a metric $h$ satisfying the Higgs equation

$$
i \Lambda F_{h}+\frac{1}{2}\left[\Phi, \Phi^{*_{h}}\right]=\frac{2 \pi}{(n-1) ! \operatorname{Vol}_{g}(X)} \mu_{g}(\mathcal{E}) \operatorname{id}_{E}
$$

Again, this result yields an isomorphism of moduli spaces: the complex geometric moduli space of stable holomorphic Higgs pairs and the differentiable geometric moduli space of irreducible integrable solutions $(A, \Phi)$ of the Higgs equation. Arbitrary solutions of this equation correspond to polystable holomorphic Higgs pairs (see [Hi], [Si]).

Holomorphic pairs. (see [Bra], [Th], [HL]) Let $E$ be a rank $r$ bundle on $X$. One wants to classify - modulo $\Gamma(X, G L(E))$ - the pairs $(\mathcal{E}, \varphi)$, where $\mathcal{E}$ is a holomorphic structure on $E$ and $\varphi$ is a holomorphic section in $\mathcal{E}$.

The stability condition for this problem depends on a real parameter $\tau$. A pair $(\mathcal{E}, \varphi)$ is $\tau$-stable if

$$
\max \left(\mu_{g}(\mathcal{E}), \sup _{\mathcal{F} \in \mathcal{R}(E)} \mu_{g}(\mathcal{F})\right)<\tau<\inf _{\substack{\mathcal{F} \in \mathcal{R}(E) \\ \varphi \in H^{0}(\mathcal{F})}} \mu_{g}(\mathcal{E} / \mathcal{F})
$$

where $\mathcal{R}(\mathcal{E})$ denotes the set of subsheaves of $\mathcal{E}$ with torsion free quotients.

There is again a differential geometric characterization of the stable pairs. $(\mathcal{E}, \varphi)$ is $\tau$-stable if and only if it is simple (i. e. it admits no non-trivial infinitesimal automorphisms) and $\mathcal{E}$ admits a metric $h$ satisfying the vortex equation

$$
i \Lambda F_{h}+\frac{1}{2} \varphi \otimes \varphi^{* h}=\frac{2 \pi}{(n-1) ! \operatorname{Vol}_{g}(X)} \tau \operatorname{id}_{E} .
$$

Witten triples. (see [W], [Dü]) Consider a line bundle $L$ on a complex surface $X$. We want to classify modulo $\Gamma(X, G L(L))=\mathcal{C}^{\infty}\left(X, \mathbb{C}^{*}\right)$ the triples $(\mathcal{L}, \varphi, \alpha)$ 
consisting of a holomorphic structure $\mathcal{L}$ on $L$, a holomorphic section $\varphi \in H^{0}(\mathcal{L})$ and a holomorphic morphism $\alpha: \mathcal{L} \rightarrow \mathcal{K}_{X}$.

The stability condition depends again on a real parameter $\tau$. A triple $(\mathcal{L}, \varphi, \alpha)$ is $\tau$-stable if either $\operatorname{deg}(\mathcal{L})<\tau$ and $\varphi \neq 0$, or $\operatorname{deg}(\mathcal{L})>\tau$ and $\alpha \neq 0$.

A triple $(\mathcal{L}, \varphi, \alpha)$ is stable if and only if it is simple (i. e. $(\varphi, \alpha) \neq 0)$ and $\mathcal{L}$ admits a metric $h$ satisfying the mixed vortex equation

$$
i \Lambda_{g} F_{h}+\frac{1}{2}\left(|\varphi|^{2}-|\alpha|^{2}\right)=\frac{2 \pi}{\operatorname{Vol}_{g}(X)} \tau .
$$

This result yields again an isomorphism of moduli spaces.

Remark: In the vortex equation and the mixed vortex equation one can replace the constant $\frac{2 \pi}{(n-1) ! V o l_{g}(X)} \tau$ on the right by a real function $t$. The corresponding stability condition depends only on $\int_{X} t v l_{g}$ when $g$ is Gauduchon. The case when $t=\frac{s_{g}}{2}$ (where $s_{g}$ stands for the scalar curvature of $g$ ) plays a crucial role in the Seiberg-Witten theory on complex surfaces (see [W], [Bi], [Dü] [OT1], [OT2], [Te2]).

Oriented holomorphic pairs. We fix again a differentiable vector bundle $E$ of rank $r$ on $X$ and a holomorphic structure $\mathcal{L}$ on $\operatorname{det} E$. This time we want to classify modulo $\Gamma(X, S L(E))$ the pairs $(\mathcal{E}, \varphi)$, where $\mathcal{E}$ is a holomorphic structure on $E$ which induces $\mathcal{L}$ on $\operatorname{det} E$ and $\varphi \in H^{0}(\mathcal{E})$ is a holomorphic section.

The stability condition in this case is (see [OST], [OT2], [Te2])

$$
\max \left(\mu_{g}(\mathcal{E}), \sup _{\mathcal{F} \in \mathcal{R}(E)} \mu_{g}(\mathcal{F})\right)<\inf _{\substack{\mathcal{F} \in \mathcal{R}(E) \\ \varphi \in H^{0}(\mathcal{F})}} \mu_{g}(\mathcal{E} / \mathcal{F}),
$$

whereas the corresponding Hermitian-Einstein type equation is

$$
i \Lambda F_{h}+\frac{1}{2}\left(\varphi \otimes \varphi^{* h}\right)_{0}=0
$$

In the case $n=r=2$, one gets a complex geometric interpretation of the moduli spaces of $P U(2)$-monopoles on complex surfaces (see [Te2]).

In order to illustrate the generality of the results in this article, consider now the following artificial moduli problem:

Fix a system of differentiable vector bundles $\left(E_{i}\right)_{0 \leq i \leq n}$ of ranks $r_{i}$, a system of holomorphic vector bundles $\left(\mathcal{F}_{j}\right)_{1 \leq j \leq m}$ of ranks $\rho_{j}$ on $X$, and a holomorphic structure $\mathcal{L}_{0}$ on $\operatorname{det} E_{0}$. 
Classify systems $\left(\mathcal{E}_{i}, \varphi_{i j}, \psi_{i}\right)$ where $\mathcal{E}_{i}$ is a holomorphic structure on $E_{i}$ such that $\mathcal{E}_{0}$ induces $\mathcal{L}_{0}$ on $\operatorname{det} E_{0}, \varphi_{i j} \in H^{0}\left(\mathcal{E}_{i}^{\vee} \otimes \mathcal{F}_{j}\right)$ are holomorphic homomorphisms, and $\psi_{i} \in \Gamma\left(X, \mathbb{P}\left(\operatorname{End}\left(\mathcal{E}_{i}\right)\right)\right.$ are holomorphic sections in the projectivizations of the endomorphism bundles of $\mathcal{E}_{i}$.

The question is: which is the correct stability (polystability) condition in this case, and which is the corresponding Hermitian-Einstein equation?

All the "classical" moduli problems listed above, as well as this artificial moduli problem are just special case of the following very general complex geometric classification problem:

A universal moduli problem. We note that in all these examples one has a system of fixed holomorphic structures, a system of variable holomorphic structures on fixed $\mathcal{C}^{\infty}$-bundles, and a system of variable sections in associated bundles, which are holomorphic with respect to both variable and fixed holomorphic structures.

The best way to formulate a universal generalization of all moduli problems of this type is the following (see [OT3], [OT5]):

Consider an exact sequence

$$
\{1\} \longrightarrow G \longrightarrow \hat{G} \longrightarrow G_{0} \longrightarrow\{1\}
$$

of complex reductive groups, and choose a $\hat{G}$-Bundle $\hat{Q}$ on $X$ and a holomorphic action $\hat{\alpha}: \hat{G} \times F \rightarrow F$ on a Kählerian manifold $F$. Let $Q_{0}:=\hat{Q} / G$ be the associated $G_{0}$-bundle and $E$ the associated $F$-bundle $E:=\hat{Q} \times_{\hat{\alpha}} F$. Fix a holomorphic structure $\mathcal{Q}_{0}$ on $Q_{0}$. Our universal classification problem is:

Classify the pairs $(\hat{\mathcal{Q}}, \varphi)$, where $\hat{\mathcal{Q}}$ is a holomorphic structure on $\hat{Q}$ inducing $\mathcal{Q}_{0}$ on $Q_{0}$ and $\varphi \in \Gamma(X, E)$ is holomorphic with respect to the holomorphic structure induced by $\hat{Q}$ on $E$, modulo the gauge group $\mathcal{G}:=\operatorname{Aut}_{Q_{0}}(\hat{Q})=\Gamma\left(X, \hat{Q} \times{ }_{\mathrm{Ad}} G\right)$.

A pair $(\hat{\mathcal{Q}}, \varphi)$ of this type will be called an oriented pair of type $\left(\hat{Q}, \hat{\alpha}, \mathcal{Q}_{0}\right)$.

This class of moduli problems was first considered in [OT3] in the case when $X$ is a complex curve; in this case the corresponding moduli spaces were used to introduce the twisted gauge theoretical Gromov-Witten invariants. The particular case $G_{0}=\{1\}$ (the case of non-oriented pairs) was previously studied by Mundet i Riera in [Mu1] on Kähler manifolds.

Example: For instance, to get our "artificial moduli problem" above as a 
special case of our universal problem, take

$$
\begin{gathered}
G:=S L\left(r_{0}, \mathbb{C}\right) \times \prod_{i=1}^{n} G L\left(r_{i}, \mathbb{C}\right), \hat{G}:=G L\left(r_{0}, \mathbb{C}\right) \times \prod_{i=1}^{n} G L\left(r_{i}, \mathbb{C}\right) \times \prod_{j=1}^{m} G L\left(\rho_{j}, \mathbb{C}\right), \\
G_{0}=\mathbb{C}^{*} \times \prod_{j=1}^{m} G L\left(\rho_{j}, \mathbb{C}\right), F:=\prod_{\substack{0 \leq i \leq n \\
1 \leq j \leq m}} \operatorname{Hom}\left(\mathbb{C}^{r_{i}}, \mathbb{C}^{\rho_{j}}\right) \times \prod_{i=0}^{n} \mathbb{P}\left(\operatorname{End}\left(\mathbb{C}^{r_{i}}\right)\right) .
\end{gathered}
$$

The action $\hat{\alpha}$ is the natural action of $\hat{G}$ on $F$, whereas the fixed holomorphic structure $\mathcal{Q}_{0}$ is defined by the system of fixed holomorphic structures $\left(\mathcal{L},\left(\mathcal{F}_{j}\right)_{1 \leq j \leq m}\right)$.

Similarly one can see easily that all classical moduli problems in our list are also special cases of this universal problem, as well as all "quiver problems" considered in $[\mathrm{AG}]$.

Our problem is to give the correct stability and polystability condition for this universal moduli problem and to characterize the polystable pairs in terms of the existence of a reduction of $\hat{\mathcal{Q}}$ to a maximal compact subgroup $\hat{K} \subset \hat{G}$ which satisfies a certain generalized Hermitian-Einstein equation. In this article, we solve this problem completely (see Theorem 3.3, Theorem 4.2 for the precise statements).

In particular, these results give a Kobayashi-Hitchin correspondence for holomorphic principal bundles with arbitrary reductive groups on arbitrary compact Hermitian manifolds.

The stability theory for holomorphic bundles with arbitrary reductive structure groups was first developed by Ramanathan and Ramanan-Ramanathan on curves $[\mathrm{Ra}],[\mathrm{RR}]$.

We mention that a Kobayashi-Hitchin correspondence for non-oriented pairs on Kähler manifolds has already been obtained in [Ba] (for linear actions) and [Mu1], whereas a very general Kobayashi-Hitchin correspondence on Kähler manifolds is stated in $[\mathrm{BGM}]^{1}$. For several important reasons we did not use the same approach and techniques as in [Mu1] and [BGM]:

- The methods used in these articles do not appear to apply to the general non-Kählerian framework.

- These articles deal only with stable objects, and it seems difficult to extend the obtained correspondence to the polystable ones using the same formalism.

\footnotetext{
${ }^{1}$ This article deals with the same type of holomorphic pairs, but the symmetry group of the problem is a more general "Lie subgroup" $\mathcal{G} \subset \operatorname{Aut}(\hat{Q})$.
} 
- It appears that the stability conditions in [Mu1] and [BGM] do not have a purely complex geometric character; more precisely, the generalized maximal weight function $\lambda$ involved in the inequality which characterizes the stability condition, also depends on a differential geometric object (namely a background $\hat{K}$-reduction of the fixed $\hat{G}$-bundle), not only on holomorphic objects.

On the other hand, the main feature of the classical stability condition for bundles is its complex geometric nature: even on general Hermitian manifolds the slope map on the set of non-trivial subsheaves of a given holomorphic bundle has a pure complex geometric character, because it does not depend on the choice of a Hermitian metric on this bundle (see [Ga], [LT]).

Our universal Kobayashi-Hitchin correspondence respects this fundamental feature of the classical Kobayashi-Hitchin correspondence, i. e. it deals only with complex geometric stability and polystability conditions.

In fact this difficulty already arises in the finite dimensional framework (see section 1): the generalized maximal weight function $\lambda$ associated with a $K$ moment map $\mu$ for a general holomorphic action $\alpha: G \times F \rightarrow F$ on a Kähler manifold is not equivariant with respect to the whole complex reductive group $G$, but only with respect to the fixed maximal compact subgroup $K$. Therefore, the inequality $\lambda(x)>0$ involved in the "analytic stability condition" is a priori not a complex geometric condition. Moreover, in the general case, it is not possible to give numerical characterizations of semistable and polystable points.

In the finite dimensional framework these difficulties have been explained and overcome in [Te2], where it was shown that one has to impose a certain completeness condition on the pair $(\alpha, \mu)$ in order to have a maximal weight function $\lambda$ with good complex geometric equivariance properties. For this class of actions (which includes all holomorphic Hamiltonian actions on compact Kähler manifolds and all linear actions) one can give complex geometric numerical characterizations of stable, semistable and polystable orbits.

Using this formalism, we were able to extend the Kobayashi-Hitchin correspondence for "universal" oriented holomorphic pairs to the case when the base manifold $X$ is an arbitrary compact Hermitian manifold. Moreover, we give a complex geometric numerical characterization not only of the stable, but also of the polystable pairs (i. e. of all pairs which admit a $\hat{K}$-reduction satisfying the generalized Hermitian-Einstein equation). This gives a complete complex geometric interpretation of the whole moduli space of solutions of the generalized Hermitian-Einstein equation.

Extending the Kobayashi-Hitchin correspondence to the non-Kählerian framework is important for many reasons: for instance this generalization furnishes complex geometric descriptions of the moduli spaces of instantons with arbitrary compact symmetry groups and of the moduli spaces of Seiberg-Witten monopoles (abelian and non-abelian) on all complex surfaces. Furthermore, an 
important application of the classical Kobayashi-Hitchin correspondence to the classification of non-Kählerian surfaces can be found in [Te1], [LT], [LYZ].

Our proof is based on the continuity method developed by Uhlenbeck and Yau for the classical Kobayashi-Hitchin correspondence (relating polystable bundles to Hermitian-Einstein connections). This seems to be the appropriate method in the non-Kählerian framework, because in this case the left hand term in the generalized Hermitian-Einstein equation is no longer a formal moment map. On the other hand, the original proof of Uhlenbeck-Yau for the classical KobayashiHitchin correspondence does not extend mechanically to our class of coupled problems with general symmetry groups, so the classical strategy developed by these authors must be completed with new techniques.

Usually moduli problems for bundles with reductive structure group $G$ are treated using a faithful representation $G \rightarrow G L(r, \mathbb{C})$ and reducing the problem to the case $G=G L(r, \mathbb{C})$, i. e. to the more familiar case of vector bundles. We will not follow this tradition, because we realized that this method does not really simplify the problem, and dealing directly with the general case is more natural and interesting. Consequently, we need an analogue of the well-known theorem of Uhlenbeck-Yau on weakly holomorphic subbundles, for general reductive structure groups $G$. In other words, we show how one can construct a meromorphic reduction of a holomorphic principal $G$-bundle $\mathcal{Q}$ to a parabolic subgroup $L \subset G$ using an $L_{1}^{2}$-section $s \in \Gamma(\operatorname{ad}(\mathcal{Q})$ which satisfies an algebraic property and a certain weak holomorphy condition. This correspondence, which follows easily from the results in [UY1], [UY2], is presented in the Appendix.

The structure of the article is the following:

We begin with the "finite dimensional" Kobayashi-Hitchin correspondence. This result gives a numerical characterization of the polystable orbits with respect to a holomorphic Hamiltonian action $(\alpha, \mu)$ satisfying our technical completeness condition. This result can be regarded as a complex geometric Hilbert criterion. A different proof (based on the method given in [Mu1] for the stable case) can be found in [Te2]. Here we give a proof which is based on the same continuity method which will be used later in the infinite dimensional gauge theoretical framework.

In the second chapter we state our "universal" moduli problem and we introduce the stability, semistability and polystability conditions for universal oriented pairs. Much care is dedicated here to the notion of degree of a meromorphic reduction to a parabolic subgroup with respect to an ad-invariant linear form; this concept is very delicate in the non-Kähler framework.

In the third chapter we introduce the Hermitian-Einstein equation for universal oriented pairs and we prove the "simple" implication of the KobayashiHitchin correspondence: any pair which admits a Hermitian-Einstein reduction is polystable. 
The fourth section deals with the "difficult" implication: any polystable pair admits an admissible Hermitian-Einstein reduction.

The Appendix contains technical results (i. e. identities for Chern connections, local diagonalization results for arbitrary compact Lie groups, meromorphic reductions to parabolic subgroups) which are of independent interest.

Acknowledgment: This work grew out as part of an ample research project on the universal Kobayashi-Hitchin correspondence and its applications, which started in Zürich in collaboration with Ch. Okonek.

\section{The finite dimensional Kobayashi-Hitchin cor- respondence}

In this section we give a brief presentation of the stability theory in the finitely dimensional Kählerian non-algebraic framework and explain the analogue of the Hilbert criterion in this framework. The Hilbert criterion we prove here gives a numerical characterization of the polystable orbits, i. e. of the orbits which intersect the vanishing locus of the moment map. Our proof - based on the continuity method - is a very good preparation for understanding the proof of our universal Kobayashi-Hitchin correspondence on Hermitian manifolds in section 4 , which will follow the same strategy but will require involved analytical techniques.

\subsection{Analytic Stability, Symplectic stability}

For complete proofs and more details about the notions and the results introduced in this section we refer to [Te2].

Let $G$ be a complex reductive group. We denote by $H(G)$ the subset $H(G) \subset \mathfrak{g}$ defined by

$$
H(G):=\{\xi \in \mathfrak{g} \mid \overline{\exp (i \mathbb{R} \xi)} \text { is compact }\}=\underset{\substack{K \subset G \\ \text { maximal compact }}}{\bigcup \mathfrak{k} .}
$$

$H(G)$ is a locally closed cone in $\mathfrak{g}$. The elements in $H(G)$ will be called vectors of Hermitian type (see [Te2]). To any $\xi \in H(G)$ one can associate a parabolic subgroup $G(\xi)$ of $G$ by

$$
G(\xi):=\left\{g \in G \mid \lim _{t \rightarrow \infty} \exp (t \xi) g \exp (-t \xi) \text { exists in } G\right\}
$$


The subgroup $G(\xi)$ fits in a short exact sequence

$$
\{1\} \longrightarrow U(\xi) \longrightarrow G(\xi) \longrightarrow Z(\xi) \longrightarrow\{1\},
$$

where

$$
U(\xi):=\left\{g \in G \mid \lim _{t \rightarrow \infty} \exp (t \xi) g \exp (-t \xi)=e\right\}
$$

is the unipotent radical of $G(\xi)$ and

$$
Z(\xi):=\left\{g \in G \mid \operatorname{ad}_{g}(\xi)=\xi\right\},
$$

is the reductive centralizer of $\xi$.

$G(\xi)$ is the direct product of its subgroups $U(\xi), Z(\xi)$. Note that that the normal subgroup $U(\xi)$ is canonically associated with the parabolic subgroup $G(\xi)$, whereas the subgroup $Z(\xi) \subset G(\xi)$ depends on the choice of the vector $\xi$.

The Lie algebras of these groups are

$$
\mathfrak{u}(\xi):=\bigoplus_{\lambda<0} \operatorname{Eig}([\xi, \cdot], \lambda), \mathfrak{z}(\xi)=z_{\mathfrak{g}}(\xi)=\operatorname{ker}([\xi, \cdot]), \mathfrak{g}(\xi)=\bigoplus_{\lambda \leq 0} \operatorname{Eig}([\xi, \cdot], \lambda)
$$

Following [Te2] we introduce in $H(G)$ the following important equivalence relation:

Definition 1.1 We say that two vectors $\xi, \zeta \in H(\mathfrak{g})$ are equivalent $(\xi \sim \zeta)$ if one of the following equivalent conditions is satisfied:

1. $\zeta \in \mathfrak{g}(\xi)$ and there exists $g \in G(\xi)$ such that $\operatorname{ad}_{g}(\xi)=\zeta$.

2. $\zeta-\xi \in \mathfrak{u}(\xi)$.

3. $\xi \in \mathfrak{g}(\zeta)$ and there exists $g \in G(\zeta)$ such that $\operatorname{ad}_{g}(\zeta)=\xi$.

4. $\xi-\zeta \in \mathfrak{u}(\zeta)$.

Proposition 1.2 Let $K \subset G$ be a maximal compact subgroup of $G$. Then the composition

$$
i \mathfrak{k} \hookrightarrow H(G) \longrightarrow H(G) / \sim
$$

is a homeomorphism.

In particular, $i \mathfrak{k}$ is a complete system of representatives for the equivalence relation $\sim$. 
Corollary 1.3 If $G$ is a closed reductive subgroup of the reductive group $\hat{G}$, then the inclusion $H(G) \rightarrow H(\hat{G})$ induces an injection

$$
H(G) / \sim_{G} \rightarrow H(\hat{G}) / \sim_{\hat{G}}
$$

which is a homeomorphism on its image.

Example: Consider the case $G=G L(r, V)$, where $V$ is a finite dimensional complex vector space. Then $H(G) \subset g l(V)$ is the subset of endomorphisms which are diagonalizable and have real eigenvalues. Every such endomorphism $\xi$ defines a filtration

$$
V(\xi)=\left(V_{\lambda}\right)_{\lambda \in \operatorname{Spec}(\xi)}, V_{\lambda}:=\bigoplus_{\nu \leq \lambda} \operatorname{Eig}(\xi, \nu)
$$

of $V$.

The parabolic subgroup $G(\xi)$ consists of those elements $g \in G L(V)$ which leave the filtration $V(\xi)$ invariant. Two elements $\xi, \zeta \in H(G)$ are equivalent if and only if they have the same eigenvalues and define the same filtration.

Let $\alpha: G \times F \rightarrow F$ be a holomorphic action of a complex reductive group on a complex manifold $F$. A Hamiltonian triple for $\alpha$ is a triple $\tau=(K, g, \mu)$ consisting of a maximal compact subgroup $K$ of $G$, a $K$-invariant Kähler metric $g$ on $F$, and a moment map $\mu$ for the induced $K$-action on $\left(F, \omega_{g}\right)$.

The data of a Hamiltonian triple for the holomorphic action $\alpha$ allows one to construct a Kählerian quotient of $F$ by $G$ (see $[\mathrm{HH}]$ ). As in the algebraic geometric GIT (where the quotient depends on the choice of a linearization of the action), a Kähler quotient depends in general essentially on the choice of the Hamiltonian triple $\tau$.

Definition 1.4 Let $\tau=(K, g, \mu)$ be Hamiltonian triple for the action $\alpha$. A point $x \in F$ will be called:

1. (symplectically) $\tau$-semistable if $\overline{G x} \cap \mu^{-1}(0) \neq \emptyset$.

2. (symplectically) $\tau$-stable if $\mathfrak{g}_{x}=\{0\}$ and $G x \cap \mu^{-1}(0) \neq \emptyset$.

3. (symplectically) $\tau$-polystable if $G x \cap \mu^{-1}(0) \neq \emptyset$.

We will denote by $F_{\tau}^{\mathrm{ss}}, F_{\tau}^{\mathrm{s}}, F_{\tau}^{\mathrm{ps}}$ the subsets of symplectically $\tau$-(semi, poly) stable points in $F$.

By the results of $[\mathrm{HH}]$ one has 
Theorem 1.5 1. $F_{\tau}^{\mathrm{ss}}$ is open in F.

2. There exists a good quotient $\pi: F_{\tau}^{\mathrm{ss}} \rightarrow Q_{\tau}$, where $Q_{\tau}$ is a Hausdorff complex space.

3. The closure of every semistable orbit contains a unique polystable orbit.

4. The inclusions $\mu^{-1}(0) \hookrightarrow F_{\tau}^{\mathrm{ps}} \hookrightarrow F_{\tau}^{\mathrm{ss}}$ induce homeomorphisms

$$
\mu^{-1}(0) / K \stackrel{\simeq}{\longrightarrow} F_{\tau}^{\mathrm{ps}} / /_{G} \simeq Q_{\tau}
$$

In particular two semistable orbits have the same image in $Q_{\tau}$ if and only if the intersection of their closures contains a polystable orbit.

Therefore the Kähler quotient $Q_{\tau}$ can identified with the symplectic quotient $\mu^{-1}(0){ }_{K}$, so this results states that, in the Kähler case, this symplectic quotient (which in general can be very singular) inherits a natural complex space structure.

Let again $\tau=(K, g, \mu)$ be a Hamiltonian triple for $\alpha$.

Remark 1.6 For any $s \in i \mathfrak{k}$, the map $t \mapsto\left\langle\mu\left(e^{t s} x\right),-i s\right\rangle$ is increasing.

Indeed the derivative of this map at $t$ is just $\left\|s_{e^{t s}}^{\#}\right\|^{2}$.

We put

$$
\lambda^{s}(x):=\lim _{t \rightarrow \infty}\left\langle\mu\left(e^{t s} x\right),-i s\right\rangle
$$

Note that

$$
\lambda^{s}(x)=\left\langle\mu\left(e^{t s} x\right),-i s\right\rangle+E_{g}\left(c_{x}^{s}\right)
$$

where $c_{x}^{s}:[0, \infty) \rightarrow F$ is the parameterized curve $t \mapsto e^{t s} x$ and $E_{g}$ stands for the energy with respect to the metric $g$.

Using the standard equivariance property of the moment map one gets the $K$-equivariance property of the map $\lambda$

$$
\lambda^{s}(x)=\lambda^{\operatorname{ad}_{k}(s)}(k x) \forall s \in i \mathfrak{k}, k \in K
$$

The following stability criterion is well-known (see for instance [Mu1], [Te2]):

Proposition 1.7 Let $x \in F$. The following conditions are equivalent

1. $x$ is symplectically $\tau$-stable.

2. $\lambda^{s}(x)>0$ for all $s \in i \mathfrak{k} \backslash\{0\}$. 
A point $x$ satisfying the second condition is usually called analytically $\tau$-stable. This criterion can be considered as the Kählerian analogue of the Hilbert criterion in GIT. However, this criterion is not satisfactory for our purposes for the following reasons:

1. The map $\lambda$ is not a purely complex geometric object; it depends on the choice of a Hamiltonian triple for the action $\alpha$, which is a differential geometric object.

2. As it stands, the analytic stability condition is not a $G$-invariant condition, because $\lambda$ is only a $K$-equivariant function, not a $G$-equivariant one.

3. The criterion does not work for semistable points: for general holomorphic actions $\alpha$ on non-compact manifolds the condition $\lambda^{s}(x) \geq 0$ for all $s \in i \mathfrak{k}$ does not imply that $x$ is symplectically semistable.

The simplest way to overcome these difficulties is to restrict ourselves to energy complete Hamiltonian triples (see [Te2]):

Definition 1.8 A Hamiltonian triple $\tau=(K, g, \mu)$ is called energy complete if for every $(s, x) \in i \mathfrak{k} \times F$ the following implication holds

$$
E_{g}\left(c_{x}^{s}\right)<\infty \Rightarrow \lim _{t \rightarrow \infty} c_{x}^{s}(t) \text { exists in } F .
$$

We refer to [Te2] for the following

Proposition 1.9 The map $\lambda: i \mathfrak{k} \times F \rightarrow \mathbb{R} \cup\{\infty\}$ associated with an energy complete Hamiltonian triple $\tau=(K, g, \mu)$ has a unique extension

$$
\lambda: H(G) \times F \rightarrow \mathbb{R} \cup\{\infty\}
$$

which is G-equivariant, i. e.

$$
\lambda^{s}(x)=\lambda^{\operatorname{ad}_{g}(s)}(g x) \forall s \in H(G) \forall x \in F \forall g \in G .
$$

This function has the following properties

1. homogeneity: $\lambda^{t s}(x)=t \lambda^{s}(x)$ for any $t \in \mathbb{R}_{\geq 0}, s \in H(G)$.

2. parabolic invariance: $\lambda^{s}(x)=\lambda^{s}(h x)$ for every $x \in F, h \in G(s)$.

3. $\sim$ invariance: $\lambda^{s}(x)=\lambda^{\sigma}(x)$ if $s \sim \sigma$.

4. semicontinuity: if $\left(x_{n}, s_{n}\right)_{n} \rightarrow(x, s)$ then $\lambda^{s}(x) \leq \liminf _{n \rightarrow \infty} \lambda^{s_{n}}\left(x_{n}\right)$. 
The $G$-equivariant extension $\lambda: H(G) \times F \rightarrow \mathbb{R} \cup\{\infty\}$ will be called (for historical reasons, see section 1.3.2) the maximal weight function associated with the (energy complete) Hamiltonian triple $\alpha$.

For our gauge theoretical results we will need the following result

Proposition 1.10 Let $G$ be a reductive normal subgroup of a reductive group $\hat{G}$, and let $\alpha: \hat{G} \times F \rightarrow F$ be a holomorphic action.

Let $K \subset \hat{K}$ be maximal compact subgroups of $G$ and $\hat{G}$ and let $\tau=(K, g, \mu)$ be an energy complete Hamiltonian triple for $\left.\alpha\right|_{G \times F}$ such that $\mu$ is $\hat{K}$-equivariant.

Then the corresponding maximal weight function $\lambda: H(G) \times F \rightarrow \mathbb{R} \cup\{\infty\}$ is $\hat{G}$-equivariant, $i$. e. it satisfies one of the following equivalent conditions:

1. $\hat{G}$-equivariance: $\lambda^{s}(x)=\lambda^{\operatorname{ad}_{g}(s)}(g x)$ for all $s \in H(G), g \in \hat{G}$.

2. $\hat{G}$-parabolic invariance: $\lambda^{s}(x)=\lambda^{s}(h x)$ for every $x \in F, h \in \hat{G}(s)$.

We denote by $G_{x}$ the stabilizer of $x \in F$, and by $\mathfrak{g}_{x}$ its Lie algebra (the infinitesimal stabilizer of $x$ ). A complex Lie subalgebra $\mathfrak{h} \subset \mathfrak{g}$ will be called reductive if it is the complexification of the Lie algebra of a compact subgroup of $G$.

Definition 1.11 Let $\alpha: G \times F \rightarrow F$ be a holomorphic a action of a complex reductive group, and let $\lambda: H(G) \times F \rightarrow \mathbb{R} \cup\{\infty\}$ be the maximal weight function associated with an energy complete Hamiltonian triple $\tau$. A point $x \in F$ will be called

1. analytically $\tau$-semistable, if $\lambda^{s}(x) \geq 0$ for every $s \in H(G)$.

2. analytically $\tau$-stable if it is $\lambda$-semistable and strict inequality $\lambda^{s}(x)>0$ holds for all $s \in H(G) \backslash\{0\}$.

3. analytically $\tau$-polystable if its infinitesimal stabilizer $\mathfrak{g}_{x}$ is reductive, it is $\lambda$-semistable and $\lambda^{s}(x)>0$ for every $s \in H(G)$ which is not equivalent to an element in $\mathfrak{g}_{x}$.

Remark 1.12 If $x$ is analytically $\tau$-semistable then

$$
\lambda^{s}(x)=\langle\mu(x),-i s\rangle=0 \forall s \in i \mathfrak{k}_{x} .
$$

Indeed, for $s \in i \mathfrak{k}_{x}$, the path $c_{x}^{s}$ is constant, hence $\lambda^{s}(x)=\langle\mu(x),-i s\rangle$. The right hand term defines a linear form on $i \mathfrak{k}_{x}$ which takes nonnegative values, hence it vanishes. 
Remark 1.13 Under the assumptions of Definition 1.11, one has:

1. $x$ is analytically $\tau$-semistable iff $\lambda^{s}(x) \geq 0$ for all $s \in i \mathfrak{k}$.

2. if $\mathfrak{g}_{x}=\mathfrak{k}_{x}^{\mathbb{C}}$, then $x$ is analytically $\tau$-polystable iff $\lambda^{s}(x)=0$ for all $s \in i \mathfrak{k}_{x}$ and $\lambda^{s}(x)>0$ for all $s \in i \mathfrak{k} \backslash i \mathfrak{k}_{x}$.

Note that if $\mathfrak{g}_{x} \neq \mathfrak{k}_{x}^{\mathbb{C}}$, one has no numerical characterization of the polystability of $x$ in terms of elements $s \in i \mathfrak{k}$; on the other hand one can always choose a Hamiltonian triple $\tau^{\prime}=\left(K^{\prime}, g^{\prime}, \mu^{\prime}\right)$ conjugated to $\tau-\mathrm{i}$. e. a triple of the form

$$
\left(K^{\prime}, g^{\prime}, \mu^{\prime}\right)=\left(\operatorname{Ad}_{\gamma}(K),\left(\gamma^{-1}\right)^{*} g, \operatorname{ad}_{\gamma^{-1}}^{t} \circ \mu \circ \gamma^{-1}\right)
$$

such that $\mathfrak{g}_{x}=\mathfrak{k}_{x}^{\prime}{ }^{\mathbb{C}}$; two conjugated triples define the same maximal weight function on $H(G) \times F$ (see [Te2]).

The comparison results in $[\mathrm{Te} 2]$ show that

Proposition 1.14 Let $\tau=(K, g, \mu)$ be an energy complete Hamiltonian triple. A point $x \in F$ is symplectically $\tau$-(semi, poly)stable if and only if it is analytically (semi, poly)stable.

This result should be regarded as a complex geometric Hilbert criterion. Note that the proof in the semistable case is rather difficult.

In the next section we will give a short proof for the polystable case, which is based on the continuity method and follows the same strategy as the proof of our universal Kobayashi-Hitchin correspondence.

\subsection{The Continuity Method in the finite dimensional case}

In this section we give an easy proof based on the continuity method for the following result:

Proposition 1.15 Let $\tau=(K, g, \mu)$ be an energy complete Hamiltonian triple and let $x \in F$. Then the following conditions are equivalent:

1. $x$ is symplectically $\tau$-polystable.

2. $x$ is analytically $\tau$-polystable

We begin with the following simple 
Lemma 1.16 Let $\mu$ be a moment map for an action $\alpha: K \times F \rightarrow F$ of a compact Lie group on a symplectic manifold $F$. Then

$$
\mu(x) \in z_{\mathfrak{k}}\left(\mathfrak{k}_{x}\right) .
$$

Proof: Regarding $\mu$ as a map $F \rightarrow \mathfrak{k}$ via an $\operatorname{ad}_{k}$-invariant inner product, we get for any $u \in \mathfrak{k}$

$$
d \mu\left(u_{x}^{\#}\right)=\left.\frac{d}{d t}\right|_{t=0} \mu\left(e^{t u}(x)\right)=\left.\frac{d}{d t}\right|_{t=0} \operatorname{ad}_{e^{t u}}(\mu(x))=[u, \mu(x)] .
$$

The left hand term vanishes when $u \in \mathfrak{k}_{x}$.

\section{Proof (of Proposition 1.15):}

We start with the simple implication $1 . \Rightarrow 2$. If $x$ is symplectically $\tau$-polystable, let $x_{0} \in G x \cap \mu^{-1}(0)$. It is easy to see that $\mathfrak{g}_{x_{0}}=\mathfrak{k}_{x_{0}}^{\mathbb{C}}$, hence $\mathfrak{g}_{x_{0}}$ (so also $\mathfrak{g}_{x}$ ) are reductive subalgebras. Moreover, one checks easily that $\lambda^{s}\left(x_{0}\right)=0$ for $s \in i \mathfrak{k}_{x_{0}}$ and $\lambda^{s}\left(x_{0}\right)>0$ for $s \in i \mathfrak{k} \backslash i \mathfrak{k}_{x_{0}}$. Therefore $x_{0}$ (so also $x$ ) is analytically $\tau$-polystable (see [Te2] for details).

Suppose now that $x$ is analytically polystable.

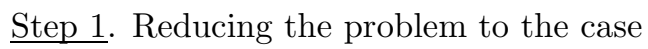

$$
\mathfrak{g}_{x} \subset z(\mathfrak{g}, G),
$$

where

$$
z(\mathfrak{g}, G):=\left\{u \in \mathfrak{g} \mid \operatorname{ad}_{g}(u)=u \forall g \in G\right\} .
$$

Note that $z(\mathfrak{g}, G)=z(\mathfrak{g})$ when $G$ is connected.

Put

$$
G^{\prime}:=Z_{G}\left(\mathfrak{g}_{x}\right)=\left\{g \in G \mid \operatorname{ad}_{g}(u)=u \forall u \in \mathfrak{g}_{x}\right\} .
$$

Since $\mathfrak{g}_{x}$ is a reductive subalgebra, it is easy to see that $G^{\prime}$ is a reductive subgroup of $G$ with Lie algebra $\mathfrak{g}^{\prime}=z_{\mathfrak{g}}\left(\mathfrak{g}_{x}\right)$.

Consider the restricted action $\alpha^{\prime}:=\left.\alpha\right|_{G^{\prime} \times F}$ of $G^{\prime}$ on $F$.

The infinitesimal stabilizer $\mathfrak{g}_{x}^{\prime}$ of $x$ with respect to $\alpha^{\prime}$ is $\mathfrak{g}_{x} \cap \mathfrak{g}^{\prime}$. Since this subalgebra of $\mathfrak{g}^{\prime}$ is contained in $\mathfrak{g}_{x}$, its element are invariant under $\operatorname{ad}_{g}$ for all $g \in G^{\prime}$, by the definition of this group. Therefore $\mathfrak{g}_{x}^{\prime} \subset z\left(\mathfrak{g}^{\prime}, G^{\prime}\right)$.

Since $\mathfrak{g}_{x}$ is a reductive subalgebra, it follows that, replacing $\tau$ by a conjugated triple if necessary, we may suppose that $\mathfrak{g}_{x}=\mathfrak{k}_{x}^{\mathbb{C}}$.

We have obviously $G^{\prime}=K^{\prime} \mathbb{C}, \mathfrak{g}^{\prime}=\mathfrak{k}^{\prime \mathbb{C}}$, where

$$
K^{\prime}:=Z_{K}\left(\mathfrak{k}_{x}\right), \mathfrak{k}^{\prime}=z_{\mathfrak{k}}\left(\mathfrak{k}_{x}\right) .
$$


The triple $\tau^{\prime}=\left(K^{\prime}, g, \mu^{\prime}:=\left.\mu\right|_{\mathfrak{k}^{\prime}}\right)$ is an energy complete Hamiltonian triple for $\alpha^{\prime}$. It is easy to see that $x$ is analytically $\tau^{\prime}$-polystable. Suppose we have proved the implication $2 . \Rightarrow 1$. in the case when (6) holds. It will follow that $x$ is symplectically $\tau^{\prime}$-polystable, hence there exists $g^{\prime} \in G^{\prime}$ such that $\mu^{\prime}\left(g^{\prime} x\right)=0$.

We state that, in our case, the relation $\mu^{\prime}\left(g^{\prime} x\right)=0$ implies the apparently stronger relation $\mu\left(g^{\prime} x\right)=0$.

Indeed, note first, that since $g^{\prime} \in G^{\prime}=Z_{G}\left(\mathfrak{g}_{x}\right)$ then $\mathfrak{g}_{g^{\prime} x}=\operatorname{ad}_{g^{\prime}}\left(\mathfrak{g}_{x}\right)=\mathfrak{g}_{x}=\mathfrak{k}_{x}^{\mathbb{C}}$.

By Lemma 1.16 we get $\mu\left(g^{\prime} x\right) \in z_{\mathfrak{k}}\left(\mathfrak{k}_{x}\right)=\mathfrak{k}^{\prime}$. But the relation $\mu^{\prime}\left(g^{\prime} x\right)=0$ means that the projection of $\mu\left(g^{\prime} x\right)$ on $\mathfrak{k}^{\prime}$ vanishes. Therefore $\mu\left(g^{\prime} x\right)=0$.

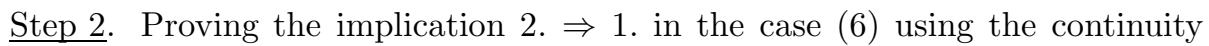
method.

Since $\mathfrak{g}_{x} \subset z(\mathfrak{g}, G)$, we have

$$
\mathfrak{k}_{g x}=\mathfrak{g}_{g x} \cap \mathfrak{k}=\operatorname{ad}_{g}\left(\mathfrak{g}_{x}\right) \cap \mathfrak{k}=\mathfrak{g}_{x} \cap \mathfrak{k}=\mathfrak{k}_{x} \forall g \in G .
$$

We seek an element $g \in G$ such that

$$
\mu(g x)=0
$$

In order to solve this equation, we use the continuity method: we choose a suitable point $x_{0} \in G x$, we solve for $\varepsilon \in(0,1]$ the perturbed equation

$$
i \mu\left(e^{s} x_{0}\right)+\varepsilon s=0, s \in i \mathfrak{k}_{x_{0}}^{\perp}
$$

and then we make $\varepsilon \rightarrow 0$.

a) We choose an element $x_{0} \in G x$ such that the perturbed equation (9) has a solution in $i \mathfrak{k}_{x_{0}}^{\perp}$ for $\varepsilon=1$.

Set $s_{1}:=-i \mu(x)$. Put $x_{0}:=e^{-s_{1}} x$. Then

$$
i \mu\left(e^{s_{1}} x_{0}\right)+s_{1}=i \mu(x)+s_{1}=0 .
$$

By Remark 1.12 we have $s_{1} \in i \mathfrak{k}_{x}^{\perp}$, so by (7), we get as required $s_{1} \in i \mathfrak{k}_{x_{0}}^{\perp}$.

b) We prove that the set

$$
T:=\left\{\varepsilon \in(0,1] \mid \exists s \in i \mathfrak{k}_{x_{0}}^{\perp} \text { such that } i \mu\left(e^{s} x_{0}\right)+\varepsilon s=0\right\}
$$

is open in $(0,1]$. 
We apply the implicit function theorem to the function

$$
l: i \mathfrak{k}_{x_{0}}^{\perp} \times(0,1] \longrightarrow i \mathfrak{k}_{x_{0}}^{\perp}, l(\varepsilon, s)=i \mu\left(e^{s} x_{0}\right)+\varepsilon s .
$$

The fact the $l$ takes values in $i \mathfrak{k}_{x_{0}}^{\perp}$ follows again from Remark 1.12. Let $s, \dot{s} \in i \mathfrak{k}_{x_{0}}^{\perp}$ and decompose

$$
\dot{s}=\lambda+[k, s], \text { with } \lambda \in i z_{\mathfrak{k}}(s), k \in z_{\mathfrak{k}}(s)^{\perp}
$$

as in the proof of Proposition 5.10 in the Appendix. Put

$$
\sigma:=\left(\left(d_{s} \exp \right)(\dot{s})\right) e^{-s}, \sigma_{h}:=p_{i \mathfrak{k}}(\sigma), \sigma_{a}:=p_{\mathfrak{k}}(\sigma) .
$$

Using the known formula (5), we get

$$
\begin{gathered}
\frac{\partial l}{\partial s}(\varepsilon, s)(\dot{s})=i d \mu\left(\sigma_{e^{s} x_{0}}^{\#}\right)+\varepsilon \dot{s}=i d \mu\left(\left(\sigma_{h}^{\#}\right)_{e^{s} x_{0}}\right)+i d \mu\left(\left(\sigma_{a}^{\#}\right)_{e^{s} x_{0}}\right)+\varepsilon \dot{s} \\
=i d \mu\left(\left(\sigma_{h}^{\#}\right)_{e^{s} x_{0}}\right)+\left[\sigma_{a}, i \mu\left(e^{s} x_{0}\right)\right]+\varepsilon \dot{s}
\end{gathered}
$$

At a pair $(\varepsilon, s)$ where $l(\varepsilon, s)=0$, this reduces to

$$
\frac{\partial l}{\partial s}(\varepsilon, s)(\dot{s})=i d \mu\left(\left(\sigma_{h}^{\#}\right)_{e^{s} x_{0}}\right)-\varepsilon\left[\sigma_{a}, s\right]+\varepsilon \dot{s} .
$$

Therefore

$$
\begin{gathered}
\left\langle\frac{\partial l}{\partial s}(\varepsilon, s)(\dot{s}), \sigma_{h}\right\rangle=\left\langle d \mu\left(\left(\sigma_{h}^{\#}\right)_{e^{s} x_{0}}\right),-i \sigma_{h}\right\rangle-\varepsilon\left\langle\left[\sigma_{a}, s\right], \sigma_{h}\right\rangle+\varepsilon\left\langle\dot{s}, \sigma_{h}\right\rangle= \\
\omega\left(-i\left(\sigma_{h}^{\#}\right)_{e^{s} x_{0}},\left(\sigma_{h}^{\#}\right)_{e^{s} x_{0}}\right)-\varepsilon\left\langle\left[\sigma_{a}, s\right], \sigma_{h}\right\rangle+\varepsilon\left\langle\dot{s}, \sigma_{h}\right\rangle \geq\left\|\left(\sigma_{h}^{\#}\right)_{e^{s} x_{0}}\right\|^{2}+\varepsilon\|\dot{s}\|^{2}
\end{gathered}
$$

by Proposition 5.10 in Appendix. Therefore $\frac{\partial l}{\partial s}(\varepsilon, s)$ is an invertible operator.

c) We prove that the set $T$ is closed in $(0,1]$.

Let $\left(\varepsilon_{n}\right)_{n}$ be a sequence converging to $\varepsilon_{0} \in(0,1]$. Taking the inner product of the equation $l\left(\varepsilon_{n}, s_{\varepsilon_{n}}\right)=0$ with $s_{n}:=s_{\varepsilon_{n}}$ we get

$$
\left\langle\mu\left(e^{s_{n}} x_{0}\right),-i s_{n}\right\rangle+\varepsilon_{n}\left\|s_{n}\right\|^{2}=0
$$

We know by Remark 1.6 that the function $t \mapsto\left\langle\mu\left(e^{t s} x_{0}\right),-i s\right\rangle$ is always increasing, so

$$
\left\|s_{n}\right\|^{2}=-\frac{1}{\varepsilon_{n}}\left\langle\mu\left(e^{s_{n}} x_{0}\right),-i s_{n}\right\rangle \leq-\frac{1}{\varepsilon_{n}}\left\langle\mu\left(x_{0}\right),-i s_{n}\right\rangle \leq \frac{1}{\varepsilon_{n}}\left\|\mu\left(x_{0}\right)\right\|\left\|s_{n}\right\|,
$$

which shows that the sequence $\left(s_{n}\right)_{n}$ is bounded. The limit of a convergent subsequence will give a solution of the equation $l\left(\varepsilon_{0}, \cdot\right)=0$. 
d) Let $\left(\varepsilon_{n}\right)_{n}$ be a sequence in $(0,1]$ with $\varepsilon_{n} \searrow 0$. We prove that any sequence $\left(s_{n}\right)_{n}, s_{n} \in i \mathfrak{k}_{x_{0}}^{\perp}$ with $l\left(\varepsilon_{n}, s_{n}\right)=0$ is bounded.

Taking a subsequence if necessary, it suffices to prove that the condition $\left\|s_{n}\right\| \rightarrow \infty$ leads to a contradiction. Put $t_{n}:=\left\|s_{n}\right\|, \sigma_{n}:=\frac{1}{t_{n}} s_{n}$. Taking a subsequence again, we may suppose that $\sigma_{n}$ converges to an element $\sigma \in i \mathfrak{k}_{x_{0}}^{\perp}$ whose norm will be of course 1. By the polystability condition, one has

$$
\lambda^{\sigma}\left(x_{0}\right)=\lim _{t \rightarrow \infty}\left\langle\mu\left(e^{t \sigma} x_{0}\right),-i \sigma\right\rangle>0,
$$

so for $t_{0}$ sufficiently large it holds

$$
\left\langle\mu\left(e^{t_{0} \sigma} x_{0}\right),-i \sigma\right\rangle>0 .
$$

Fix such a $t_{0}$. For all $n$ sufficiently large we will still have

$$
\left\langle\mu\left(e^{t_{0} \sigma_{n}} x_{0}\right),-i \sigma_{n}\right\rangle>0
$$

Assuming $t_{n} \rightarrow \infty$, we can find $n$ with the above property such that also $t_{n} \geq t_{0}$. By the monotony property Remark 1.6 we would get

$$
0<\left\langle\mu\left(e^{t_{n} \sigma_{n}} x_{0}\right),-i \sigma_{n}\right\rangle=\frac{1}{t_{n}}\left\langle\mu\left(e^{s_{n}} x_{0}\right),-i s_{n}\right\rangle=-\frac{1}{t_{n}} \varepsilon_{n}\left\|s_{n}\right\|^{2},
$$

which is obviously a contradiction.

Now it suffices to note that the limit of a convergent subsequence of $\left(s_{n}\right)_{n}$ will be a solution of the equation $\mu\left(e^{s} x_{0}\right)=0$.

\subsection{Maximal weight functions for linear and projective actions}

The explicit form of the maximal weight function associated with a linear or projective actions is well known (see for instance [Mu1]). We explain below this computation for completeness.

\subsubsection{Linear actions}

Let $\rho: G \rightarrow G L(V)$ be a rational linear representation of a connected reductive group $G$ on a finite dimensional complex vector space $V$. 
Let $K$ be a maximal compact subgroup of $G$ with a fixed invariant inner product on its Lie algebra $\mathfrak{k}$, and let $g$ be a $K$-invariant Hermitian inner product on $V$. One has a standard moment map for the $K$ action which is given by

$$
\mu_{0}(v)=\rho^{*}\left(-\frac{i}{2} v \otimes v^{*}\right),
$$

and any other moment map has the form

$$
\mu_{\tau}=\mu_{0}-i \tau,
$$

where $\tau \in i z(\mathfrak{k})$.

Let $\xi \in i \mathfrak{k}$ and let $V=\oplus_{j=1}^{k} V_{j}$ be the decomposition of $V$ into eigenspaces of $\xi$. In other words $\left.\rho_{*}(\xi)\right|_{V_{j}}=\xi_{j} \operatorname{id}_{V_{j}}$, where $\xi_{j}$ are the pairwise distinct eigenvalues of $\xi$.

Put

$$
V_{\xi}^{ \pm}:=\bigoplus_{ \pm \xi_{j}>0} V_{j}, V_{ \pm}^{\xi}:=\bigoplus_{ \pm \xi_{j} \geq 0} V_{j}
$$

Let $v \in V$. Decompose $v$ as $v=\sum_{j} v_{j}$ with $v_{j} \in V_{j}$.

One gets

$$
\lambda_{\tau}^{\xi}(v):=\lim _{t \rightarrow \infty}\left\langle\mu_{\tau}\left(\rho\left(e^{t \xi}\right) v\right),-i \xi\right\rangle=\left\{\begin{array}{cc}
+\infty & \text { if } \exists j \in\{1, \ldots, k\} \text { such that } \\
& \xi_{j}>0 \text { and } v_{j} \neq 0, \\
\langle\tau, \xi\rangle & \text { otherwise }
\end{array}\right.
$$

Suppose for simplicity that $\operatorname{ker}\left(\rho_{*}\right)=\{0\}$.

In this case we conclude that a vector $v \in V$ is $\tau$-stable if and only if for every $\xi \in i \mathfrak{k} \backslash\{0\}$ with $\langle\tau, \xi\rangle \leq 0$ one has $\operatorname{pr}_{V_{\xi}^{+}}(v) \neq 0$.

Therefore, the non-stable locus is

$$
N S_{\tau}=\bigcup_{\langle\tau, \xi\rangle \leq 0, \xi \neq 0} V_{-}^{\xi}
$$

Let us describe this set in the case when $G$ is a complex torus. Decompose $V$ as

$$
V=\bigoplus_{\chi \in R} V_{\chi}
$$

where $R \subset \operatorname{Hom}(i \mathfrak{k}, \mathbb{R}) \subset \mathfrak{g}^{\vee}$ is the finite set of weights of the representation $\rho$, and $V_{\chi}:=\left\{v \in V \mid \rho_{*}(\gamma)(v)=\chi(\gamma) v \forall \gamma \in \mathfrak{g}\right\}$. 
For every subset $A \subset R$ put

$$
V_{A}:=\bigoplus_{\chi \in A} V_{\chi}
$$

Since $G$ is a torus, the space $V_{-}^{\xi}$ associated with any $\xi \in \mathfrak{i k}$ has the form $V_{A}$ for some $A \subset R$. Define

$C_{A}:=\left\{\xi \in i \mathfrak{k} \mid V_{-}^{\xi}=V_{A}\right\}=\{\xi \in i \mathfrak{k} \mid \chi(\xi) \geq 0 \forall \chi \in A, \chi(\xi)>0 \forall \chi \in R \backslash A\}$.

Note that the sets $C_{A}$ give a partition of $i \mathfrak{k}$ in pairwise disjoint polyhedral convex cones. Using $(*)$ we get

$$
N S_{\tau}=\bigcup_{C_{A} \cap\left(H_{\tau} \backslash\{0\}\right) \geq 0 \neq \emptyset} V_{A},
$$

where $H_{\bar{\tau}}^{\geq 0}$ denotes the half-space defined by the inequality $\langle\tau, \xi\rangle \geq 0$.

Corollary 1.17 Suppose that $G$ is a torus. Then there exists a finite set $\Phi_{\tau} \subset$ $i \mathfrak{k}$ such that the following conditions become equivalent:

1. $v$ is $\tau$-stable,

2. $\lambda_{\tau}^{\xi}(v)>0$ for all $\xi \in \Phi_{\tau}$,

3. $v \notin \bigcup_{\xi \in \Phi} V_{-}^{\xi}$.

Proof: The non-empty intersections $C_{A} \cap\left(H_{\tau} \backslash\{0\}\right)$ define a finite partition of the pointed half-space $H_{\bar{\tau}}^{\geq 0} \backslash\{0\}$. Take a point in every set of this partition.

\subsubsection{Projective actions}

Let $\rho: G \rightarrow G L(V)$ be a rational linear representation of a complex reductive group $G$, and let $\alpha: G \times \mathbb{P}(V) \rightarrow \mathbb{P}(V)$ be the induced projective action.

The Fubini-Study Kähler form on $\mathbb{P}(V)$ associated with a Hermitian metric $h$ on $V$ is $\frac{i}{2 \pi} \partial \bar{\partial} \log \|\cdot\|^{2}=\frac{1}{4 \pi} d d^{c} \log \|\cdot\|^{2}$.

Let $K \subset G$ be a maximal compact subgroup which acts isometrically on the Hermitian space $(V, h)$. The standard moment map for the $K$-action on $\mathbb{P}(V)$ is (see $[\mathrm{Ki}]$ )

$$
\mu([v])=\rho^{*}\left[-\frac{i}{2 \pi} \frac{v \otimes \bar{v}}{\|v\|^{2}}\right]
$$


The maximal weight of the Hermitian endomorphism $f \in \operatorname{Herm}(V)$ with respect to a vector $v \in V$ is defined

$$
\lambda_{m}(f, v):=\max \left\{\lambda \in \operatorname{Spec}(f) \mid \operatorname{pr}_{\operatorname{Eig}(f, \lambda)}(v) \neq 0\right\} .
$$

If $f$ is associated with a one parameter subgroup of $G L(V)$ (i. e. it has the form $d_{1} \varphi(1)$, where $\left.\varphi: \mathbb{C}^{*} \rightarrow G L(V) \in \operatorname{Hom}\left(\mathbb{C}^{*}, G L(V)\right)\right)$, then all its eigenvalues are integers, so in this case $\lambda_{m}(f, \cdot)$ takes integer values. In this case we put $\lambda_{m}(\varphi, \cdot):=\lambda_{m}\left(d_{1} \varphi(1), \cdot\right)$. Writing

$$
\varphi(\zeta)=\sum_{n \in R(\varphi)} \zeta^{n} \operatorname{id}_{V_{n}^{\varphi}}
$$

with $V=\bigoplus_{n \in R(\varphi)} V_{n}^{\varphi}$, we see that

$$
\lambda_{m}(\varphi,[v])=\max \left\{n \in R(\varphi) \mid \operatorname{pr}_{V_{n}^{\varphi}}(v) \neq 0\right\},
$$

which is just the maximal weight of $\varphi$ with respect to $[v]$ occurring in the algebraic geometric Hilbert criterion.

Let $\xi \in i \mathfrak{k}$. One has

$$
\begin{aligned}
\lambda^{\xi}([v]):=\lim _{t \rightarrow \infty}\left\langle\mu\left(\rho\left(e^{t \xi}\right) v\right)\right. & , i \xi\rangle=\frac{1}{2 \pi} \lim _{t \rightarrow \infty}\left\langle\left[\frac{e^{t \xi} v \otimes \overline{e^{t \xi} v}}{\left\|e^{t \xi} v\right\|^{2}}\right], \rho_{*}(\xi)\right\rangle= \\
= & \frac{1}{2 \pi} \lambda_{m}\left(\rho_{*}(\xi), v\right)
\end{aligned}
$$

Therefore, up to a positive constant, $\lambda^{\xi}([v])$ is just the maximal weight of the Hermitian endomorphism $\rho_{*}(\xi)$ with respect to $[v]$.

The point is that, for a rational representation $\rho$, the analytic (semi)stability of a point $[v] \in \mathbb{P}(V)$ can be checked by verifying the corresponding inequality only for one-parameter subgroups $\mathbb{C}^{*} \rightarrow G$ (see [Ki]). In other words, in this case one has

Analytic Stability $=$ GIT Stability $=$ Symplectic Stability.

\section{A "universal" complex geometric classifica- tion problem}

\subsection{Oriented holomorphic pairs}

Let

$$
1 \longrightarrow G \stackrel{j}{\longrightarrow} \hat{G} \longrightarrow G_{0} \longrightarrow 1
$$


be an exact sequence of complex reductive Lie groups; the closed normal subgroup $G$ of $\hat{G}$ will be called the symmetry group of the variables and the quotient group $G_{0}$ will be called the symmetry group of parameters (see the discussion in the Introduction).

Let $X$ be a connected compact complex manifold, $\hat{Q}$ a differentiable principal $\hat{G}$-bundle over $X$, and set $Q_{0}:=\hat{Q} \times{ }_{\hat{G}} G_{0}$. Let $F$ be a Kähler manifold and $\hat{\alpha}$ a holomorphic action of $\hat{G}$ on $F$. Consider the associated bundle

$$
E:=\hat{Q} \times_{\hat{G}} F .
$$

We fix a holomorphic structure $J_{0}$ on $Q_{0}$, and we denote by $\mathcal{Q}_{0}$ the resulting holomorphic bundle. We state the following universal complex geometric classification problem:

Classify the pairs $(\hat{J}, \varphi)$, where $\hat{J}$ is a holomorphic structure on the bundle $\hat{Q}$ which induces $J_{0}$ on $Q_{0}$ and $\varphi$ is a $\hat{J}$-holomorphic section in $E$. The classification is considered up to isomorphy defined by the natural action of the complex gauge group

$$
\mathcal{G}:=\operatorname{Aut}_{Q_{0}}(\hat{Q})=\Gamma\left(X, \hat{Q} \times \operatorname{Ad}_{\hat{G}} G\right) .
$$

A pair $(\hat{J}, \varphi)$ as above will be called a holomorphic pair of type $\left(\hat{Q}, J_{0}, \hat{\alpha}\right)$.

A possible answer to the classification problem above can be given by restricting our attention to the subspace of simple pairs. The concept of simple pair can be defined in our general framework as follows:

For any pair $(\hat{J}, \varphi)$ we define the space of its infinitesimal automorphisms (its infinitesimal stabilizer) by

$$
\mathfrak{g}_{\hat{J}, \varphi}:=\left\{v \in A^{0}\left(\hat{Q} \times_{\text {ad }} \mathfrak{g}\right) \mid \bar{\partial}_{\hat{J}} v=0, v^{\#} \circ \varphi=0\right\}
$$

where $v^{\#}$ denotes the vertical vector field on $E$ defined by $v$.

Definition 2.1 The pair $(\hat{J}, \varphi)$ is called simple if $\mathfrak{g}_{\hat{J}, \varphi}=\{0\}$.

Using non-linear versions of the methods of [LO], [LT] one should be able to endow the moduli space of simple holomorphic pairs with the structure of a (possibly non-Hausdorff, possibly singular) complex orbifold (see [Su] for the case of standard holomorphic pairs).

A more involved approach to the classification problem is based on the concept of stability. The data of a Hermitian metric $g$ on $X$ allows us to introduce a concept of stability for holomorphic pairs of a fixed type $\left(\hat{Q}, J_{0}, \hat{\alpha}\right)$, and to construct the corresponding moduli space of $g$-stable pairs. This moduli space will be a Hausdorff open subspace in the moduli orbifold of simple pairs. 
Remark: In algebraic geometry and classical complex geometry one avoids using differential geometric objects (as is our fixed differentiable bundle $\hat{Q}$ ), so it is worth to note that there is a purely complex geometric (algebraic geometric) equivalent formulation of our classification problem.

Fix an algebra morphism $\hat{\mathfrak{c}}: H^{*}(B \hat{G}, \mathbb{Z}) \rightarrow H^{*}(X, \mathbb{Z})$. Classify the triples $(\hat{\mathcal{Q}}, \iota, \varphi)$, where $\hat{\mathcal{Q}}$ is a holomorphic $\hat{G}$ bundle on $X$ with fixed characteristic morphism $\hat{\mathfrak{c}}, \iota$ is a holomorphic isomorphism $\iota: \hat{\mathcal{Q}} \times{ }_{\hat{G}} G_{0} \rightarrow \mathcal{Q}_{0}$ and $\varphi$ is a holomorphic section of $\hat{\mathcal{Q}} \times{ }_{\hat{G}} F$. The classification is considered up to isomorphism of holomorphic $\hat{G}$-bundles.

A triple $(\hat{\mathcal{Q}}, \iota, \varphi)$ as above will be called a holomorphic triple of type $\left(\hat{\mathfrak{c}}, \mathcal{Q}_{0}, \hat{\alpha}\right)$. The algebraic version can be obtained by replacing everywhere the word "holomorphic" by "algebraic". This terminology was used in [OST].

One can prove easily that a "moduli set" of holomorphic triples of a fixed type is a discrete disjoint union of "moduli sets" of oriented holomorphic pairs.

\subsection{The stability condition for universal oriented holomor- phic pairs}

\subsubsection{The degree of a meromorphic $L$-reduction with respect to an ad-invariant linear form}

Let $G$ be a complex reductive group, and let $L$ be a parabolic subgroup of $G$. When $G$ is connected it follows that $L$ has the form $L=G(s)$, where $s \in H(G)$. In the general case we will consider only parabolic subgroups of the the form $G(s)$ so, for us, a parabolic subgroup will always be associated to an element in $s \in H(G)$. We know that $L$ fits in the exact sequence

$$
1 \rightarrow U(L) \rightarrow L \rightarrow Z(L) \rightarrow 1
$$

which is canonically associated with $L, i$. e. it does not depend on the presentation $L=G(s) . U(L)$ is just the unipotent radical of $L$, and $Z(L)$ is the canonical reductive quotient of $L$.

Let $\psi: \mathfrak{l} \rightarrow \mathbb{C}$ be an $\operatorname{ad}_{L}$-invariant linear form. Such a form must be a Lie-algebra morphism ${ }^{2}$, i. e. it must fulfill

$$
\psi([a, b])=0 \forall a, b \in \mathfrak{l} .
$$

Recall that

$$
\mathfrak{u}(L)=\mathfrak{u}(s):=\bigoplus_{\lambda<0} \operatorname{Eig}([s, \cdot], \lambda), \mathfrak{z}(L)=\mathfrak{z}(s)=z_{\mathfrak{g}}(s),
$$

\footnotetext{
${ }^{2}$ For a connected Lie group, the converse is also true: a $\mathbb{C}$-valued Lie algebra morphism is an ad-invariant form.
} 
and that $[s, \mathfrak{u}(s)]=\mathfrak{u}(s)$. This shows that $\left.\psi\right|_{\mathfrak{u}(s)}=0$. Therefore,

Remark 2.2 Any $\operatorname{ad}_{L}$-invariant linear form $\psi: \mathfrak{l} \rightarrow \mathbb{C}$ is induced by an $\operatorname{ad}_{Z(L)}{ }^{-}$ invariant linear form $\mathfrak{z}(L) \rightarrow \mathbb{C}$ on its canonical reductive quotient.

Let now $Z$ by any complex reductive group. Let $C$ be a maximal compact subgroup of $Z$ and $T \subset C$ a maximal torus. Consider the root decomposition

$$
\mathfrak{z}=\mathfrak{t}^{\mathbb{C}} \oplus\left(\bigoplus_{r \in R \backslash\{0\}} \mathfrak{z}_{r}\right)
$$

of the Lie algebra $\mathfrak{z}$. For any $r \in R \backslash\{0\}$, one has obviously $\mathfrak{z}_{r} \subset\left[\mathfrak{t}^{\mathbb{C}}, \mathfrak{z}_{r}\right]$. Therefore,

Remark 2.3 If $\psi: \mathfrak{z} \rightarrow \mathbb{C}$ is an $\operatorname{ad}_{Z}$-invariant form, then $\left.\psi\right|_{\mathfrak{z}_{r}}=0$ for every $r \in R \backslash\{0\}$.

Definition 2.4 An ad-invariant form $\psi: \mathfrak{z} \rightarrow \mathbb{C}$ will be called real if for any (and hence for every) maximal compact subgroup $C$ of $Z$, the restriction $\left.\psi\right|_{i \mathrm{c}}$ takes real values.

Definition 2.5 A nondegenerate $\operatorname{ad}_{G}$-invariant symmetric bilinear form

$$
h: \mathfrak{g} \times \mathfrak{g} \rightarrow \mathbb{C}
$$

will be called invariant complex inner product of Euclidean type if it induces an Euclidean inner product on any (and hence on all) subspaces of the form $i \mathfrak{k}$, where $\mathfrak{k}$ is the Lie algebra of a maximal compact subgroup $K$.

In the semisimple case, the Killing form is such an invariant complex inner product of Euclidean type. We can always find such an complex inner product by choosing an $\operatorname{ad}_{K}$-invariant real inner product on any subspace $i \mathfrak{k}$ and extending it in a $\mathbb{C}$-bilinear way. However, this notion should be regarded as a complex geometric one (which does not require a distinguished maximal compact subgroup).

Example: Let $h$ be an invariant complex inner product of Euclidean type on $\mathfrak{g}$. Any element $s \in H(G)$ defines a real $\operatorname{ad}_{G(s)}$-invariant form $h(s): \mathfrak{g}(s) \rightarrow \mathbb{C}$ given by $u \mapsto h(s, u)$.

Note that the restriction of $h$ to $\mathfrak{g}(s)$ is not non-degenerate. Its kernel is $\mathfrak{u}(s)$. Indeed, for any $u, v \in \mathfrak{g}(s)$, one has

$$
h(u, v)=h\left(\operatorname{ad}_{\exp (t s)}(u), \operatorname{ad}_{\exp (t s)}(v)\right)=h\left(\operatorname{pr}_{\mathfrak{z}(s)}(u), \operatorname{pr}_{\mathfrak{z}(s)}(v)\right),
$$


because $\operatorname{ad}_{\exp (t s)}(u) \rightarrow \operatorname{pr}_{\mathfrak{z}(s)}(u)$ as $t \rightarrow \infty$. Therefore, the formula for $h(s)$ reads

$$
h(s)(u)=h\left(s, \operatorname{pr}_{\mathfrak{z}(s)}(u)\right),
$$

which is obviously $\operatorname{ad}_{G(s)}$-invariant, because $G(s) \rightarrow Z(s)$ is a group morphism, $h$ is ad-invariant, and $s$ is $\operatorname{ad}_{Z(s)}$-invariant.

By the Chern Weil theory, any $\operatorname{ad}_{z}$-invariant linear form $\psi$ on its Lie algebra $\mathfrak{z}$ defines a characteristic class $c_{\psi}(W)$ in the 2 -cohomology of the base of any $Z$-bundle $W$. More precisely, if $A$ is a a connection on $W$, one sets

$$
c_{\psi}(W)=\left[\psi\left(\frac{i}{2 \pi} F_{A}\right)\right]_{\mathrm{DR}} .
$$

For a $L$-bundle $\Lambda$ we put

$$
c_{\psi}(\Lambda):=c_{\psi}\left(\Lambda \times_{L} Z(L)\right) .
$$

Definition 2.6 The degree of a $Z$-bundle $W$ (respectively of a L-bundle $\Lambda$ ) on a compact $n$-dimensional Kähler manifold $(X, g)$ with respect to the real adinvariant form $\psi: \mathfrak{z} \rightarrow \mathbb{C}$ (respectively $\psi: \mathfrak{z}(L) \rightarrow \mathbb{C}$ ) is defined by

$$
\operatorname{deg}_{g}(W, \psi):=\left\langle c_{\psi}(W) \cup\left[\omega_{g}\right]^{n-1},[X]\right\rangle\left(\operatorname{deg}_{g}(\Lambda, \psi):=\operatorname{deg}_{g}\left(\Lambda \times_{L} Z(L)\right)\right)
$$

In the non-Kähler case, one can still define the degree with respect to $\psi$ of a holomorphic $Z$ bundle ( $L$-bundle), but the obtained invariant will no longer be a topological invariant. One has to use the same strategy as in the well known case of line bundles on Gauduchon manifolds.

Let $g$ be a Gauduchon metric on $X$ and let $\mathcal{W}$ be a holomorphic $Z$-bundle on $X$. Choose a maximal compact subgroup $C$ of $Z$, an $C$-reduction $R$ of $\mathcal{W}$ and let $A_{R}$ its Chern connection (see section 5.1). The point is that, as in the case of line bundles, the following important result holds:

Lemma 2.7 The real closed 2-form $\psi\left(\frac{i}{2 \pi} F_{A_{R}}\right)$ is independent of the chosen $C$ -

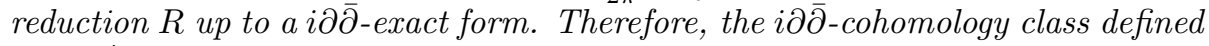
by $\psi\left(\frac{i}{2 \pi} F_{A_{R}}\right)$ depends only on the holomorphic $Z$-bundle $\mathcal{W}$.

Proof: Let $R_{0}$ and $R$ be two $C$-reductions of $\mathcal{W}$. We can write

$$
R=e^{-\frac{s}{2}}\left(R_{0}\right),
$$

where $s \in A^{0}\left(R_{0} \times{ }_{\text {ad }} i \mathfrak{c}\right)$. By Corollary 5.3, one has

$$
F_{A_{R}}=F_{A_{R_{0}}}+\bar{\partial}\left(e^{-s} \partial_{A_{R_{0}}}\left(e^{s}\right)\right),
$$


It is easy to see that

$$
\psi\left(e^{-s} \partial_{A_{R_{0}}}\left(e^{s}\right)\right)=\partial(\psi(s)) .
$$

This follows easily using Proposition 5.12 and formula (72), taking into account Remark 2.3. Therefore

$$
\psi\left(F_{A_{R}}\right)=\psi\left(F_{A_{R_{0}}}\right)+\bar{\partial} \partial \psi(s) .
$$

This allows us to define

Definition 2.8 Let $\mathcal{W}$ be a holomorphic $Z$-bundle, $\mathcal{L}$ a holomorphic L-bundle on a compact $n$-dimensional Gauduchon manifold $(X, g)$, and let $\psi: \mathfrak{z} \rightarrow \mathbb{C}$ (respectively $\psi: \mathfrak{z}(L) \rightarrow \mathbb{C}$ ) be a real ad-invariant form. Let $C$ be a maximal compact subgroup of $Z$ (respectively $Z(L)$ ). We define

$$
\begin{gathered}
c_{\psi}(\mathcal{W})=\left[\psi\left(\frac{i}{2 \pi} F_{A_{R}}\right)\right] \in Z^{1,1}(X, \mathbb{R}) / i \partial \bar{\partial} A^{0}(X, \mathbb{R}), c_{\psi}(\mathcal{L}):=c_{\psi}\left(\mathcal{L} \times_{L} Z(L)\right) \\
\operatorname{deg}(\mathcal{W}, \psi):=\left\langle c_{\psi}(\mathcal{W}), \omega_{g}^{n-1}\right\rangle=\int_{X} \psi\left(\frac{i}{2 \pi} F_{A_{R}}\right) \wedge \omega_{g}^{n-1} \\
\operatorname{deg}(\mathcal{L}, \psi):=\operatorname{deg}\left(\mathcal{L} \times_{L} Z(L), \psi\right)
\end{gathered}
$$

where $A_{R}$ is the Chern connection of any $C$-reduction of $\mathcal{W}\left(\right.$ of $\mathcal{L} \otimes_{L} Z(L)$ ).

We will need an extension of the degree map to meromorphic $L$-reductions of a holomorphic $G$-bundle. We begin with the following

Definition 2.9 Let $L$ be a parabolic subgroup of a complex reductive group $G$ and $\mathcal{Q}$ a holomorphic $G$-principal bundle over a complex manifold $X$. A meromorphic reduction of $G$ to $L$ is a meromorphic section in the associated bundle

$$
\mathcal{Q}_{L}:=\mathcal{Q} / L=\mathcal{Q} \times{ }_{G}(G / L),
$$

i. e. a closed reduced irreducible subspace $\rho \subset \mathcal{Q}_{L}$ which generically projects isomorphically onto $X$ via the bundle projection $q_{L}: \mathcal{Q}_{L} \rightarrow X$.

Since the fibre $G / L$ of $\mathcal{Q}_{L}$ is a projective manifold, it follows easily that the maximal open subset $X_{\rho} \subset X$ over which $\rho$ is the graph of a holomorphic section is the complement of a Zariski closed subset of codimension at least 2. On $X_{\rho}$ $\rho$ defines a holomorphic $L$-reduction $\left.\mathcal{Q}^{\rho} \subset \mathcal{Q}\right|_{X_{\rho}}$. 
Example: Let $\mathcal{Q}$ be a holomorphic $G L(r, \mathbb{C})$-bundle and $\mathcal{E}$ the associated holomorphic vector bundle. The data of a meromorphic reduction of $\mathcal{Q}$ to the parabolic group

$$
L:=\left\{\left(\begin{array}{cc}
A & B \\
0 & C
\end{array}\right) \mid A \in G L(p, \mathbb{C}), B \in M(p, r-p), C \in G L(r-p, \mathbb{C})\right\}
$$

is equivalent to the data of a rank $p$ subsheaf $\mathcal{F} \subset \mathcal{E}$ with torsion free quotient $\mathcal{H}$. Such a subsheaf is necessarily reflexive and defines a subbundle on the subset $X \backslash \operatorname{Sing}(\mathcal{H})$. Given such a subsheaf $\mathcal{F}$, the corresponding meromorphic section $\rho$ is given by

$$
\rho:=\overline{\operatorname{im}(f)}
$$

where $f: X \backslash \operatorname{Sing}(\mathcal{H}) \rightarrow \mathbb{G} r_{p}(\mathcal{E})$ is defined by the holomorphic subbundle $\left.\mathcal{F}\right|_{X \backslash \operatorname{Sing}(\mathcal{H})}$ of $\left.\mathcal{E}\right|_{X \backslash \operatorname{Sing}(\mathcal{H})}$.

Suppose now that $\mathcal{Q}$ is a holomorphic $G$-bundle on the compact Gauduchon manifold $(X, g), L$ a parabolic subgroup of $G, \rho \subset \mathcal{Q}_{L}$ a meromorphic $L$ reduction of $\mathcal{Q}$ and $\psi: \mathfrak{z}(L) \rightarrow \mathbb{C}$ a real $\operatorname{ad}_{Z(L)}$-invariant form. We denote by $q_{L}: \mathcal{Q}_{L} \rightarrow X$ the natural projection and by $\iota_{\rho}: \rho \hookrightarrow \mathcal{Q}_{L}$ the inclusion map.

We claim that the assignment

$$
A^{1,1}\left(\mathcal{Q}_{L}\right) \ni \varphi \mapsto \int_{\rho \backslash \operatorname{Sing}(\rho)} \varphi \wedge q_{L}^{*}\left(\omega_{g}^{n-1}\right)
$$

defines a $\partial \bar{\partial}$-closed current of bidimension $(1,1)$ on $\mathcal{Q}_{L}$. Indeed, let $\mathfrak{R} \stackrel{\alpha}{\longrightarrow} \rho$ any desingularization of $\rho$. Then the assignment

$$
A^{1,1}\left(\mathcal{Q}_{L}\right) \ni \varphi \mapsto \int_{\mathfrak{R}}\left(\iota_{\rho} \circ \alpha\right)^{*}(\varphi) \wedge\left(q_{L} \circ \alpha\right)^{*}\left(\omega_{g}^{n-1}\right)
$$

obviously defines a $\partial \bar{\partial}$-closed current of bidimension $(1,1)$ on $\mathcal{Q}_{L}$, because the pull-back $\left(q_{L} \circ \alpha\right)^{*}\left(\omega_{g}^{n-1}\right)$ is a $\partial \bar{\partial}$-closed form on the smooth manifold $\Re$.

But the assignment (11) coincides with (10) - which is independent of the desingularization $\alpha$-because $\rho \backslash \operatorname{Sing}(\rho)$ is identified via $\alpha$ with an open subset of total measure of $\mathfrak{R}$.

We will denote the current defined by the formulae (10), (11) by $\left.q_{L}^{*}\left(\omega_{g}^{n-1}\right)\right|_{\rho}$.

Since, by definition, $\mathcal{Q}_{L}$ is the base of the holomorphic $L$-bundle $\mathcal{Q} \rightarrow \mathcal{Q}_{L}$, we can define

\section{Definition 2.10}

$$
\operatorname{deg}(\rho, \psi):=\left\langle c_{\psi}\left(\mathcal{Q} \rightarrow \mathcal{Q}_{L}\right),\left.q_{L}^{*}\left(\omega_{g}^{n-1}\right)\right|_{\rho}\right\rangle .
$$


In the presence of a $K$-reduction $P \subset \mathcal{Q}$ of the $G$-bundle $\mathcal{Q}$, one can give an explicit formula for the degree map.

Note first that the intersection $K_{L}:=L \cap K$ projects isomorphically onto a maximal compact subgroup of $Z(L)$ via the canonical epimorphism $L \rightarrow Z(L)$. This follows from formula (80) in Appendix.

Therefore, $P \rightarrow \mathcal{Q}_{L} \simeq P / K_{L}$ can be regarded as a reduction of the associated $Z(L)$-bundle $\mathcal{W}:=\mathcal{Q} \times_{L} Z(L) \rightarrow \mathcal{Q}_{L}$ to its maximal compact subgroup $K_{L}$.

It is not difficult to express the Chern connection $B$ of the $K_{L}$-bundle $P \rightarrow \mathcal{Q}_{L}$ in terms of the Chern connection $A$ of the original $K$-bundle $P \rightarrow X$. Denoting by $\omega_{A}, \omega_{B}$ the corresponding connection forms, one has

$$
\omega_{B}=\operatorname{pr}_{\mathfrak{l} \cap \mathfrak{k}}\left(\omega_{A}\right)
$$

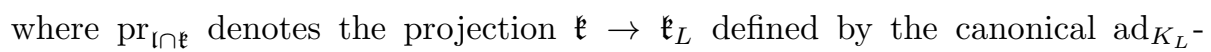
invariant decomposition

$$
\mathfrak{k}=\mathfrak{k}_{L} \oplus \mathfrak{k}_{L}^{\perp},
$$

where $\mathfrak{k}_{L}^{\perp}:=\operatorname{pr}_{\mathfrak{k}}(\mathfrak{u}(L))$. Therefore, on $P$ one can write

$$
\omega_{A}=\omega_{B}+\alpha, \operatorname{pr}_{\mathfrak{k}_{L}} \Omega_{A}=\Omega_{B}+\frac{1}{2} \operatorname{pr}_{\mathfrak{k}_{L}}[\alpha \wedge \alpha],
$$

where $\alpha \in A^{1}\left(P, \mathfrak{k}_{L}^{\perp}\right)$ is the $\mathfrak{k}_{L}^{\perp}$-component of $\omega_{A}$. Regarding the forms in the second formula as bundle valued forms on the base $Q_{L}$ one gets

$$
\operatorname{pr}_{P \times_{K_{L}} \mathfrak{k}_{L}}\left[q_{L}^{*}\left(F_{A}\right)\right]=F_{B}+\frac{1}{2} \operatorname{pr}_{P \times_{K_{L}} \mathfrak{k}_{L}}[a \wedge a],
$$

where $a \in A^{1}\left(\mathcal{Q}_{L}, P \times_{K_{L}} \mathfrak{k}_{L}^{\perp}\right)$ is the bundle valued 1-form on $\mathcal{Q}_{L}$ associated with $\alpha$.

Using the definitions above, we get

$$
\operatorname{deg}(\rho, \psi)=\int_{\rho \backslash \operatorname{Sing}(\rho)} \psi\left(\frac{i}{2 \pi} F_{B}\right) \wedge q_{L}^{*}\left(\omega_{g}^{n-1}\right)=\int_{X_{\rho}} \psi\left(\frac{i}{2 \pi} \rho^{*}\left(F_{B}\right)\right) \wedge \omega_{g}^{n-1},
$$

where $\rho^{*}$ is the pull-back associated with the holomorphic map $X_{\rho} \rightarrow \mathcal{Q}_{L}$ defined by $\rho$. The point is that the form $\rho^{*}\left(F_{B}\right)$ is just the curvature of the connection $A_{\rho}$ induced by $A$ on the $K_{L}$-subbundle $\left.Q^{\rho} \cap P \subset P\right|_{X_{\rho}}$, whereas $a_{\rho}:=\rho^{*}(a)$ is just the second fundamental form of this subbundle. Therefore we get the following

Proposition 2.11 If $g$ be a Gauduchon metric on $X$, then

$$
\operatorname{deg}_{g}(\rho, \psi)=\int_{X} \psi\left(\frac{i}{2 \pi} F_{A_{\rho}}\right) \wedge \omega_{g}^{n-1}
$$


where $A_{\rho}$ is the connection induced by the Chern connection $A$ of any $K$ reduction $P \subset \mathcal{Q}$ on the $K_{L}$-subbundle $P^{\rho}:=\left.Q^{\rho} \cap P \subset P\right|_{X_{\rho}}$.

Using the canonical decomposition $\mathfrak{g}=\mathfrak{z}(L) \oplus \mathfrak{z}(L)^{\perp}$ obtained by complexifying the decomposition (12), one can extend $\psi$ in a natural way to a linear form $\psi: \mathfrak{g} \rightarrow \mathbb{C}$ (which of course is not $\operatorname{ad}_{G}$-invariant in general).

Therefore the degree formula becomes

$$
\operatorname{deg}_{g}(\rho, \psi)=\int_{X}\left[\psi\left(\frac{i}{2 \pi} F_{A}\right)-\psi\left(\frac{i}{4 \pi}\left[a_{\rho} \wedge a_{\rho}\right]\right)\right] \wedge \omega_{g}^{n-1},
$$

where $a_{\rho}$ is the second fundamental form of $Q^{\rho} \cap P$ in $\left.P\right|_{X_{\rho}}$.

The degree map can be extended to the case of an arbitrary Hermitian metric $g$ in the following way (see [LT] for the case of vector bundles):

Recall first that the maximum principle holds for the operator $P=i \Lambda_{g} \bar{\partial} \partial$ for any Hermitian metric $g$. Consequently, one gets a direct sum decomposition

$$
\mathcal{C}^{\infty}(X, \mathbb{R})=\mathbb{R} \oplus P\left(\mathcal{C}^{\infty}(X, \mathbb{R})\right)
$$

which is $L^{2}$-orthogonal when $g$ is Gauduchon. Dualizing, we get a direct sum decomposition

$$
\mathcal{D}^{\prime}(X, \mathbb{R})=\mathbb{R} \oplus P\left(\mathcal{D}^{\prime}(X, \mathbb{R})\right)
$$

Using this decomposition, we define the operator $I_{g}: \mathcal{D}^{\prime}(X, \mathbb{R}) \rightarrow \mathbb{R}$ (the generalized integral) by

$$
I_{g}(\varphi):=\operatorname{Vol}_{g}(X) \operatorname{pr}_{\mathbb{R}}(\varphi) .
$$

$I_{g}$ vanishes always on $\operatorname{im}(P)$ and coincides with the standard integral when $g$ is Gauduchon.

Remark 2.12 (The Hermitian case) Let $g$ be a Hermitian metric. Replacing the usual integral by the operator $I_{g}$ in the formulae (13), (14) one gets a well defined holomorphic invariant $\operatorname{deg}_{g}(\rho, \psi)$ for pairs $(\rho, \psi)$ consisting of a real ad-invariant linear form $\psi$ on $\mathfrak{z}(\mathfrak{l})$ and a meromorphic reduction $\rho$ of $\mathcal{Q}$ to the parabolic subgroup $L$.

The degree with respect to a Hermitian metric $g$ coincides with the degree with respect to the unique Gauduchon metric $g^{\prime}$ in its conformal class defined by the condition $\int_{X}\left(g^{\prime} / g\right)^{n-1} \operatorname{vol}_{g}=\operatorname{Vol}_{g}(X)$. Therefore, the properties of the degree map associated with general Hermitian metrics reduce easily to the Gauduchon case. 


\subsubsection{Stability, Semistability, Polystability}

We come back to our gauge theoretical problem, so consider a differentiable principal $\hat{G}$-bundle $\hat{Q}$ on a compact Hermitian manifold $(X, g)$.

Let $\hat{\alpha}: \hat{G} \times F \rightarrow F$ be a holomorphic action, $G$ a closed normal subgroup of $\hat{G}$. We choose a Kähler structure $g_{F}$ on $F$, a maximal compact subgroup $K$ of $G$ acting isometrically on $\left(F, g_{F}\right)$, a maximal compact subgroup $\hat{K}$ of $\hat{G}$ which contains $K$, and a $\hat{K}$-equivariant moment map $\mu$ for the induced $K$-action. We will suppose that the Hamiltonian triple $\sigma=\left(K, g_{F}, \mu\right)$ is energy complete, so that it defines a generalized maximal weight function $\lambda: F \times H(G) \rightarrow \mathbb{R} \cup\{\infty\}$ with the fundamental properties listed in Proposition 1.9 and 1.10. We also recall that $\lambda$ depends only on the conjugacy class of $\sigma$ (see section 1.1).

Let $E$ be the associated bundle $E:=\hat{Q} \times{ }_{\hat{G}} F$. Consider again $\zeta \in H(G)$ and a $\hat{G}(\zeta)$-reduction $\left.\hat{Q}^{\rho} \subset \hat{Q}\right|_{X_{\rho}}$ defined on an open subset $X_{\rho} \subset X$. For points $x \in X_{\rho}, e \in E_{x}$ we put

$$
\lambda^{\zeta}(e, \rho):=\lambda^{\zeta}\left(\varphi_{q}(e)\right),
$$

where $\varphi_{q}: E_{x} \rightarrow F$ is the holomorphic isomorphism defined by an element $q \in \hat{Q}_{x}^{\rho}$. By Proposition 1.10 it follows that the term on the right does not depend on the choice of $q \in \hat{Q}_{x}^{\rho}$.

We fix an ad-invariant complex inner product $h$ of Euclidean type on $\hat{\mathfrak{g}}$ (see Definition 2.5).

Definition 2.13 Fix a holomorphic structure $J_{0}$ on $Q_{0}$. A holomorphic pair $(\hat{J}, \varphi)$ of type $\left(\hat{Q}, J_{0}, \hat{\alpha}\right)$ is called $\sigma$-semistable if, for every $\xi \in H(G)$ and for every meromorphic reduction $\rho$ of the holomorphic bundle $\hat{\mathcal{Q}}_{\hat{J}}$ to $\hat{G}(\xi)$ it holds

$$
\frac{2 \pi}{(n-1) !} \operatorname{deg}_{g}(\rho, h(\xi))+\int_{X_{\rho}} \lambda^{\xi}(\varphi, \rho) \operatorname{vol}_{g} \geq 0 .
$$

A pair $(\hat{J}, \varphi)$ of type $\left(\hat{Q}, J_{0}, \hat{\alpha}\right)$ is called $\sigma$-stable if it is $\sigma$-semistable and strict inequality holds for any $\xi \in H(G) \backslash\{0\}$.

The left hand term in the inequality in Definition 2.13 will be called the total maximal weight of the pair $(\hat{J}, \varphi)$ with respect to the pair $(\xi, \rho)$.

Remark 2.14 In general, the (semi)stability condition depends on the Hermitian metric $g$, the Hamiltonian triple $\sigma=\left(K, g_{F}, \mu\right)$ and the invariant complex inner product of Euclidean type $h$.

Note that, for any $\hat{g} \in \hat{G}$, the translation $R_{\hat{g}}: \hat{\mathcal{Q}} \rightarrow \hat{\mathcal{Q}}$ defines a biholomorphic diffeomorphism $\hat{\mathcal{Q}} / \hat{G}(\psi) \rightarrow \hat{\mathcal{Q}} / \hat{G}\left(\operatorname{ad}_{\hat{g}^{-1}}(\psi)\right)$, hence it assigns to a $\hat{G}(\psi)$ 
meromorphic reduction $\rho$ of $\hat{\mathcal{Q}}$ a $\hat{G}\left(\operatorname{ad}_{g^{-1}}(\psi)\right)$ meromorphic reduction $\rho \hat{g}$. The corresponding total maximal weights of a pair $(\hat{J}, \varphi)$ coincide, so we get the following important

Remark 2.15 It suffices to check the (semi)stability condition for a single representative in every equivalence class of $H(G)$ modulo the adjoint action $\operatorname{ad}_{\hat{G}}$ of the group $\hat{G}$.

In particular it suffices to check this inequality for any element $\psi \in i C$, where $C \subset \mathfrak{t}$ is a closed Weyl chamber in the Lie algebra $\mathfrak{t}$ of a maximal torus of a maximal compact subgroup $K \subset G$.

We recall that a Lie subalgebra $\mathfrak{b} \subset \mathfrak{g}$ is called reductive if it is the complexification of the Lie algebra of a compact subgroup of $G$.

Definition 2.16 Let $\hat{Q}$ be a $\hat{G}$-bundle on a connected compact complex manifold $X$. A finite dimensional Lie subalgebra $\mathfrak{u} \subset A^{0}\left(\hat{Q} \times_{\text {ad }} \mathfrak{g}\right)$ will be called reductive if there exists a reductive Lie subalgebra $\mathfrak{b} \subset \mathfrak{g}$ and a $Z_{\hat{G}}(\mathfrak{b})$-reduction $\hat{Z} \hookrightarrow \hat{Q}$ of $\hat{Q}$ such that $\mathfrak{u}$ is the image of $\mathfrak{b}$ via the obvious morphism

$$
\mathfrak{b} \hookrightarrow A^{0}\left(\hat{Z} \times{ }_{\mathrm{ad}} \mathfrak{b}\right) \rightarrow A^{0}\left(\hat{Z} \times{ }_{\mathrm{ad}} \mathfrak{g}\right)=A^{0}(\hat{Q} \times \text { ad } \mathfrak{g})
$$

Such a reduction gives an isomorphism $-\hat{Z}: \mathfrak{b} \rightarrow \mathfrak{u}$. Note that the reduction $\hat{Z}$ can be recovered from the isomorphism $b_{\hat{Z}}$, because

$$
\hat{Z}=\left\{q \in \hat{Q} \mid b_{\hat{Z}}(q)=b \forall b \in \mathfrak{b}\right\} .
$$

In particular one has

Remark 2.17 If $\hat{\mathcal{Q}}$ is a holomorphic $\hat{G}$-bundle on $X$ and $\mathfrak{u} \subset H^{0}(X, \hat{\mathcal{Q}} \times$ ad $\mathfrak{g})$ is a reductive Lie subalgebra consisting of holomorphic sections, then any $Z_{\hat{G}}(\mathfrak{b})$ reduction $\hat{Z} \subset \hat{\mathcal{Q}}$ which induces an isomorphism $\mathfrak{b} \rightarrow \mathfrak{u}$ is holomorphic.

Definition 2.18 A pair $(\hat{J}, \varphi)$ of type $\left(\hat{Q}, J_{0}, \hat{\alpha}\right)$ is called $\sigma$-polystable if:

1. its infinitesimal stabilizer $\mathfrak{g}_{\hat{J}, \varphi}$ is a reductive subalgebra of $A^{0}(\hat{Q} \times$ ad $\mathfrak{g})$,

2. it is $\sigma$-semistable and the equality

$$
\frac{2 \pi}{(n-1) !} \operatorname{deg}_{g}(\rho, h(\xi))+\int_{X_{\rho}} \lambda^{\xi}(\varphi, \rho) \text { vol }_{g}=0
$$


in the semistability condition holds if and only if $\rho$ is induced by a global holomorphic $Z_{\hat{G}}(\xi)$-reduction $\hat{\mathcal{Z}}(\xi) \hookrightarrow \hat{\mathcal{Q}}_{\hat{J}}$, and the section

$$
\xi_{\hat{\mathcal{Z}}(\xi)} \in H^{0}\left(X, \hat{\mathcal{Q}}_{\hat{J}} \times_{\text {ad }} \mathfrak{g}\right)
$$

defined by $\xi$ via this reduction belongs to the infinitesimal stabilizer $\mathfrak{g}_{\hat{J}, \varphi}$.

Definition 2.19 Let $z(\mathfrak{g}, \hat{G}) \subset z(\mathfrak{g})$ be the centralizer of $\hat{G}$ in $\mathfrak{g}$, i. e. the subalgebra consisting of those elements of $\mathfrak{g}$ which are fixed under the adjoint $\hat{G}$-action. In other words, $z(\mathfrak{g}, \hat{G})$ is the Lie algebra of $Z(G, \hat{G}):=G \cap Z(\hat{G})$.

When $\hat{G}$ is connected, one has $z(\mathfrak{g}, \hat{G})=z(\mathfrak{g})$. Note that $z(\mathfrak{g}, \hat{G})$ can always be identified with a finite dimensional abelian subalgebra of $A^{0}(\hat{Q} \times$ ad $\mathfrak{g})$. For pairs $(\hat{J}, \varphi)$ with infinitesimal stabilizer contained in $z(\mathfrak{g}, \hat{G})$ the polystability condition becomes:

Remark 2.20 Let $\beta=(\hat{J}, \varphi)$ be a pair with infinitesimal stabilizer $\mathfrak{g}_{\beta}$ contained in $z(\mathfrak{g}, \hat{G})$. Then $\beta$ is polystable if and only if it is semistable and the inequality in the semistability condition is strict unless $\xi \in H(G) \cap \mathfrak{g}_{\beta}$, in which case one must have $\hat{G}(\xi)=\hat{G}, \hat{\mathcal{Q}}_{\hat{J}}^{\rho}=\hat{\mathcal{Q}}_{\hat{J}}$.

We show now that, by a suitable reduction of the structure group of the bundle, one can reduce the study of any polystable pair $\beta=(\hat{J}, \varphi)$ to the case $\mathfrak{g}_{\beta} \subset z(\mathfrak{g}, \hat{G})$ (compare with Step 1 in the proof of Theorem 1.15 in the finite dimensional framework).

Let $\beta$ be a $\sigma$-polystable pair, $\mathfrak{b} \subset \mathfrak{g}$ a reductive subalgebra and $\hat{Z} \subset \hat{Q}$ a $Z_{\hat{G}}(\mathfrak{b})$-reduction of $\hat{Q}$ such that the infinitesimal stabilizer $\mathfrak{g}_{\beta}$ is identified with $\mathfrak{b}$ via this reduction (see Definition 2.16). Put

$$
\hat{G}^{\prime}:=Z_{\hat{G}}(\mathfrak{b}), G^{\prime}:=Z_{G}(\mathfrak{b})=\hat{G}^{\prime} \cap G, G_{0}^{\prime}:=\hat{G}^{\prime} /{ }_{G^{\prime}} \subset G_{0}, \hat{\alpha}^{\prime}:=\left.\hat{\alpha}\right|_{\hat{G}^{\prime} \times F} .
$$

By Remark 2.17 it follows that $\hat{Z}$ is a holomorphic reduction of $\hat{\mathcal{Q}}_{\hat{J}}$; we denote by $\hat{J}^{\prime}$ the induced holomorphic structure.

Put $Z_{0}:=\hat{Z} \times{\hat{G^{\prime}}}^{\prime} G_{0}^{\prime}$, and note that $Z_{0}$ is a holomorphic $G_{0}^{\prime}$-subbundle of $\mathcal{Q}_{0}$, so it has an induced holomorphic structure $J_{0}^{\prime}$.

The restriction of the maximal weight function $\lambda: H(G) \times F \rightarrow \mathbb{R} \cup\{\infty\}$ to $H\left(G^{\prime}\right) \times F \rightarrow \mathbb{R} \cup\{\infty\}$ is the maximal weight function $\lambda^{\prime}$ of a suitable Hamiltonian triple $\left(K^{\prime}, g^{\prime}, \mu^{\prime}\right)$ for the restricted action $\alpha^{\prime}:=\left.\alpha\right|_{F \times \hat{G}^{\prime}}$, where $\mu^{\prime}$ is equivariant with respect to a maximal compact subgroup $\hat{K}^{\prime} \supset K^{\prime}$ of $\hat{G}^{\prime}$. 
Proposition 2.21 If $\beta=(\hat{J}, \varphi)$ is $\sigma$-polystable, then the associated pair $\beta^{\prime}:=$ $\left(\hat{J}^{\prime}, \varphi\right)$ is a $\sigma^{\prime}$-polystable pair of type $\left(\hat{Z}, J_{0}^{\prime}, \hat{\alpha}^{\prime}\right)$. The infinitesimal stabilizer $\mathfrak{g}_{\beta^{\prime}}^{\prime}$ of this pair is $\mathfrak{b} \cap z_{\mathfrak{g}}(\mathfrak{b})=z(\mathfrak{b})$ which is contained in the centralizer $z\left(\mathfrak{g}^{\prime}, \hat{G}^{\prime}\right)$.

Proof: Consider the holomorphic bundles $\hat{\mathcal{Q}}:=\hat{\mathcal{Q}}_{\hat{J}}, \hat{\mathcal{Z}}:=\hat{\mathcal{Z}}_{\hat{J}^{\prime}}$ defined by the holomorphic structures $\hat{J}, \hat{J}^{\prime}$. It is easy to see that $\left(\hat{J}^{\prime}, \varphi\right)$ is $\sigma^{\prime}$-semistable because, for any $s \in H\left(G^{\prime}\right)$ any meromorphic $\hat{G}^{\prime}(s)$-reduction $\rho^{\prime}$ of $\hat{\mathcal{Z}}$ extends to a meromorphic $\hat{G}(s)$-reduction $\rho$ of $\hat{\mathcal{Q}}$, and the two total maximal weights coincide.

The delicate part is the fact that $\beta^{\prime}$ is $\sigma^{\prime}$-polystable. Let $\rho^{\prime}$ be a meromorphic $\hat{G}^{\prime}(s)$-reduction of $\hat{\mathcal{Z}}$ such that the corresponding total maximal weight vanishes, and let $\rho$ be the associated $\hat{G}(s)$-reduction of $\hat{\mathcal{Q}}$. We know that $\rho$ is induced by a global holomorphic $Z_{\hat{G}}(s)$-reduction $\mathcal{Z}(s) \subset \hat{\mathcal{Q}}$ such that the section $\sigma:=s_{\hat{\mathcal{Z}}(s)}$ defined by $s$ via this reduction belongs to the infinitesimal stabilizer $\mathfrak{g}_{\beta}$. We claim that, under these assumptions, one has

1. $s$ coincides with the element $b \in \mathfrak{b}$ defined by the equality $\sigma=b_{\hat{\mathcal{Z}}}$.

2. $\hat{G}^{\prime}(s)=\hat{G}^{\prime}$, in particular the meromorphic reduction $\rho^{\prime}$ is just $\hat{\mathcal{Z}} \stackrel{\equiv}{\hookrightarrow} \hat{\mathcal{Z}}$.

In order to prove this, note first that

$$
\hat{\mathcal{Z}}(s)=\{q \in \hat{\mathcal{Q}} \mid \sigma(q)=s\} .
$$

On the other hand, $\left.\left.\hat{\mathcal{Z}}(s)\right|_{X_{\rho}} \subset \hat{\mathcal{Q}}^{\rho} \subset \hat{\mathcal{Z}}\right|_{X_{\rho}} \cdot \hat{G}(s)$, because, by construction, $\hat{\mathcal{Q}}^{\rho}$ is the $\hat{G}(s)$-saturation of a $\hat{G}^{\prime}(s)$-bundle contained in $\hat{\mathcal{Z}}$. Since $X_{\rho}$ is dense, the inclusion $\hat{\mathcal{Z}}(s) \subset \hat{\mathcal{Z}} \cdot \hat{G}(s)$ holds on whole $X$. Therefore, any $q \in \hat{Z}(s)$ has the form $z g$ with $z \in \hat{\mathcal{Z}}$ and $g \in \hat{G}(s)$. We get, for any $q \in \hat{\mathcal{Z}}(s)$

$$
s=\sigma(q)=\sigma(z g)=\operatorname{ad}_{g^{-1}}(\sigma(z))=\operatorname{ad}_{g^{-1}}(b),
$$

where $b \in \mathfrak{b}$ is the element which corresponds to $\sigma \in \mathfrak{g}_{\hat{J}, \varphi}$ via the reduction $\hat{\mathcal{Z}}$.

Therefore $b=\operatorname{ad}_{g}(s)$ with $g \in \hat{G}(s)$. Now recall that $\operatorname{ad}_{\hat{G}(s)}(s)=s+\mathfrak{u}(s)$. But $b \in z_{\hat{\mathfrak{g}}}(s)$ (because $\left.s \in z_{\hat{\mathfrak{g}}}(\mathfrak{b})\right)$. We get $b=s$. The second statement follows from the first.

\section{$3 \quad$ Hermitian-Einstein pairs}

\subsection{The Hermitian-Einstein equation}

Choose a maximal compact subgroup $K$ of $G$ and a maximal compact subgroup $\hat{K}$ of $\hat{G}$ which contains $K$. Let $\hat{P} \subset \hat{Q}$ be a $\hat{K}$-reduction of $\hat{Q}$, and let $P_{0}$ be 
the bundle associated with $\hat{P}$ with structure group $K_{0}:=\hat{K} / K$. Consider the (unique) integrable connection $A_{0} \in \mathcal{A}^{1,1}\left(P_{0}\right)$ which corresponds to $J_{0}$ via the Chern correspondence. Using the Chern correspondence (see section 5.1), our classification problem associated with the complex data $\left(\hat{Q}, J_{0}, \alpha\right)$ can be reformulated as follows:

Classify the pairs $(\hat{A}, \varphi)$, where $\hat{A}$ is an integrable connection on $\hat{P}$ which induces a fixed integrable connection $A_{0}$ on $P_{0}$, and $\varphi$ is a $J_{A}$-holomorphic section in the associated bundle $E=\hat{P} \times{ }_{\hat{K}} F$. The classification is considered up to isomorphy defined by the action of the complex gauge group

$$
\mathcal{G}:=\Gamma\left(X, \hat{P} \times \operatorname{Ad}_{\hat{K}} G\right)=\operatorname{Aut}_{Q_{0}}(\hat{Q}) .
$$

This action is induced - via the Chern correspondence - by the $\mathcal{G}$-action on the space of holomorphic pairs.

A pair $(\hat{A}, \varphi)$ as above will be called an integrable pair of type $\left(\hat{P}, A_{0}, \alpha\right)$.

Note that, formally, $\mathcal{G}$ can be regarded as the complexification of the real gauge group

$$
\mathcal{K}:=\Gamma\left(X, \hat{P} \times \operatorname{Ad}_{\hat{K}} K\right)=\operatorname{Aut}_{P_{0}}(\hat{P}) .
$$

We denote by $\mathcal{A}_{A_{0}}(\hat{P})$ the space of connections on $\hat{P}$ which induce $A_{0}$ on $P_{0}$.

Recall that we have fixed an ad-invariant complex inner product of Euclidean type $h$ on $\hat{\mathfrak{g}}$ (see Definition 2.5). This inner product defines an orthogonal projection $\operatorname{pr}_{i \mathfrak{k}}: i \hat{\mathfrak{k}} \rightarrow i \mathfrak{k}$ which induces a bundle projection

$$
\hat{P} \times{ }_{\text {ad }} i \hat{\mathfrak{k}} \longrightarrow \hat{P} \times{ }_{\text {ad }} i \mathfrak{k}
$$

which will be denoted by the same symbol.

Using the restriction of $h$ to $\mathfrak{k}$, we can view $\mu$ as a map with values in $\mathfrak{k}$.

Definition 3.1 An pair $(\hat{A}, \varphi)$ of type $\left(\hat{P}, A_{0}, \hat{\alpha}\right)$ is called $\mu$-Hermitian-Einstein if it is integrable and solves the equation

$$
\operatorname{pr}_{i \mathfrak{k}}\left[i \Lambda_{g} F_{\hat{A}}\right]+i \mu(\varphi)=0,
$$

which will be called the generalized Hermitian-Einstein equation associated with the data $\left(\hat{P}, A_{0}, \hat{\alpha}, \mu, g\right)$.

Let $(\hat{J}, \varphi)$ be a holomorphic pair of type $\left(\hat{Q}, J_{0}, \hat{\alpha}\right) . A \hat{K}$-reduction $\hat{P}$ of $\hat{Q}$ will be called $\mu$-Hermitian-Einstein if the associated pair $\left(A_{\hat{P}, \hat{J}}, \varphi\right)$ is $\mu$-HermitianEinstein.

Here we denoted by $A_{\hat{P}, \hat{J}}$ the Chern connection of the pair $(\hat{P}, \hat{J})$ (see section 5.1). 
Note that this equation is $\mathcal{G}$-invariant, so that one can consider the moduli space of solutions

$$
\mathcal{M}^{\mathrm{HE}}:=\left[\mathcal{A}_{A_{0}}(\hat{P}) \times \Gamma(E)\right]^{\mathrm{HE}} / \mathcal{G},
$$

where $\left[\mathcal{A}_{A_{0}}(\hat{P}) \times \Gamma(E)\right]^{\mathrm{HE}} \subset\left[\mathcal{A}_{A_{0}}(\hat{P}) \times \Gamma(E)\right]$ is the subspace of (integrable) pairs satisfying the equation (HE).

Note that any section $v \in \Gamma\left(X, \hat{Q} \times{ }_{\text {ad }} \mathfrak{g}\right)$ induces a section $v^{\#}$ in the vertical tangent bundle $V_{E}$ of $E$.

Definition 3.2 A pair $(\hat{A}, \varphi)$ of type $\left(\hat{P}, A_{0}, \alpha\right)$ is called irreducible if its infinitesimal stabilizer

$$
\mathfrak{k}_{(\hat{A}, \varphi)}:=\left\{v \in A^{0}\left(\hat{P} \times_{\text {ad }} \mathfrak{k}\right) \mid \nabla_{\hat{A}} v=0, v^{\#} \circ \varphi=0\right\}
$$

(which is a sub-Lie-algebra of $\left.A^{0}\left(\hat{P} \times_{\text {ad }} \mathfrak{k}\right)\right)$ vanishes.

\subsection{Pairs which allow Hermitian-Einstein reductions are polystable}

We come back to the assumptions and notations of section 2.2.2. The aim of this section is to prove the following

Theorem 3.3 Let $(\hat{J}, \varphi)$ be a holomorphic pair of type $\left(\hat{Q}, J_{0}, \alpha\right)$. Suppose that there exists a $\hat{K}$-reduction $\hat{P} \subset \hat{Q}$ such that the associated pair $\left(A_{\hat{P}, \hat{J}}, \varphi\right)$ is $\mu$-Hermitian-Einstein. Then $(\hat{J}, \varphi)$ is $\sigma$-polystable, and it is stable if only if $\left(\hat{A}_{\hat{P}, \hat{J}}, \varphi\right)$ is irreducible.

\section{Proof:}

We will assume that $g$ is Gauduchon (see Remark 2.12). We check first the inequality in the definition of $\sigma$-semistability. By Remark 2.15 it suffices to check this inequality for elements $\xi \in i \mathfrak{k}$.

Let $\rho$ be a meromorphic reduction of $\hat{Q}$ to $\hat{G}(\xi)$, and put $\hat{P}^{\rho}:=\hat{Q}^{\rho} \cap \hat{P}$. The inclusion $\iota:\left.\hat{P}^{\rho} \hookrightarrow \hat{P}\right|_{X_{\rho}}$ defines a reduction of the bundle $\left.\hat{P}\right|_{X_{\rho}}$ to the subgroup $\hat{K}(\xi):=\hat{G}(\xi) \cap \hat{K}$ of $K$. It is easy to see that

$$
\hat{K}(\xi)^{\mathbb{C}}=Z_{\hat{G}}(\xi) .
$$

Therefore $\xi$ is invariant under the structure group of $\hat{P}^{\rho}$, so it defines a section $s(\rho, \xi)$ in $A^{0}\left(\left.\hat{P}\right|_{X_{\rho}} \times_{\text {ad }} i \mathfrak{k}\right)$. 
Put $\hat{A}:=A_{\hat{P}, \hat{J}}$. We get

$$
\begin{gathered}
0=\int_{X_{\rho}}\left\langle\operatorname{pr}_{i \mathfrak{k}}\left[i \Lambda_{g} F_{\hat{A}}\right]+i \mu(\varphi), s(\rho, \xi)\right\rangle \operatorname{vol}_{g}= \\
=\int_{X_{\rho}}\left\langle i \Lambda_{g} F_{\hat{A}}, s(\rho, \xi)\right\rangle v l_{g}+\int_{X_{\rho}} i \mu(\varphi)(s(\rho, \xi)) v l_{g} .
\end{gathered}
$$

Recall now that the functions $t \mapsto \mu_{\xi}\left(e^{t \xi}\right)$ involved in the definition of $\lambda$ are monotonely increasing, so that, in every point $x \in X_{\rho}$ we get

$$
i \mu(\varphi)(s(\rho, \xi))(x) \leq \lambda^{s(\rho, \xi)}(\varphi)(x) .
$$

This shows that

$$
\int_{X_{\rho}} i \mu(\varphi)(s(\rho, \xi)) \operatorname{vol}_{g} \leq \int_{X_{\rho}} \lambda^{s(\rho, \xi)}(\varphi) \operatorname{vol}_{g}=\int_{X_{\rho}} \lambda^{\xi}(\varphi) \operatorname{vol}_{g},
$$

with equality if and only if $s(\rho, \xi)^{\#} \circ \varphi=0$ over $X_{\rho}$.

The adjoint bundle $\hat{P} \times{ }_{\text {ad }} \hat{\mathfrak{k}}=\hat{P}^{\rho} \times$ ad $\hat{\mathfrak{k}}$ of $\hat{P}$ splits as

$$
\hat{P}^{\rho} \times \text { ad } \hat{\mathfrak{k}}=\operatorname{ad}\left(\hat{P}^{\rho}\right) \oplus\left[\hat{P}^{\rho} \times \text { ad }[\hat{\mathfrak{k}}(\xi)]^{\perp}\right] .
$$

The connection $\hat{A}$ on $\hat{P}$ induces a connection $\hat{A}_{\rho}$ on $\hat{P}^{\rho}$ whose horizontal space at a point $p \in \hat{P}^{\rho}$ is the intersection

$$
\left[H_{\hat{A}, p} \oplus p \cdot[\hat{\mathfrak{k}}(\xi)]^{\perp}\right] \cap T_{p}\left(\hat{P}^{\rho}\right)
$$

where $H_{\hat{A}, p}$ stands here for the $\hat{A}$-horizontal space. The connection form of this connection is

$$
\omega_{\hat{A}_{\rho}}=\operatorname{pr}_{\operatorname{ad}\left(\hat{P}^{\rho}\right)} \iota^{*}\left(\omega_{\hat{A}}\right)
$$

The difference $a:=\hat{A}-\iota_{*}\left(\hat{A}_{\rho}\right)$ is a section of the subbundle $\hat{P}^{\rho} \times$ ad $[\hat{\mathfrak{k}}(\xi)]^{\perp}$ of $\operatorname{ad}(\hat{P})$ (the second fundamental form of the subbundle $\hat{P}^{\rho}$ of $\hat{P}$ with respect to the connection $\hat{A})$.

Decompose the Lie algebra $\hat{\mathfrak{g}}$ as

$$
\hat{\mathfrak{g}}=\hat{\mathfrak{g}}_{+} \oplus \hat{\mathfrak{g}}_{-} \oplus \hat{\mathfrak{g}}_{0}
$$

where $\hat{\mathfrak{g}}_{ \pm}$is direct sum of the eigenspaces of the endomorphism

$$
[\xi, \cdot] \in \operatorname{End}(\hat{\mathfrak{g}})
$$


corresponding to the positive (negative) eigenvalues, and $\hat{\mathfrak{g}}_{0}=\operatorname{ker}[\xi, \cdot]$. Note that

$$
\hat{\mathfrak{g}}_{0}=[\hat{\mathfrak{k}}(\xi)]^{\mathbb{C}}, \hat{\mathfrak{g}}_{-} \oplus \hat{\mathfrak{g}}_{0}=\hat{\mathfrak{g}}(\xi), \hat{\mathfrak{g}}_{+}=[\hat{\mathfrak{k}}(\xi)]^{\perp}, \hat{\mathfrak{g}}_{+}=\overline{\hat{\mathfrak{g}}}_{-}
$$

where the conjugation in the last formula is taken with respect to the real structure $\hat{\mathfrak{g}}=i \hat{\mathfrak{k}} \otimes \mathbb{C}$ on $\hat{\mathfrak{g}}$.

Therefore, the second fundamental form $a$ decomposes as

$$
a=a^{+}+a^{-}
$$

where $a_{ \pm} \in A^{1}\left(X, \hat{P}^{\rho} \times_{\text {ad }} \mathfrak{g}_{ \pm}\right)$and

$$
a^{-}=-\bar{a}^{+} .
$$

But $\hat{\mathfrak{g}}_{+}=T_{[e]}[\hat{G} / \hat{G}(\xi)]$ and on $X_{\rho}$

$$
\hat{P}^{\rho} \times_{\mathrm{ad}} \hat{\mathfrak{g}}_{+}=\hat{Q}^{\rho} \times_{\mathrm{ad}} \hat{\mathfrak{g}}_{+}=\rho^{*}\left(T^{V}\left(\hat{Q} \times_{\hat{G}}\left[\hat{G}_{/} \hat{G}_{\xi}\right]\right)\right),
$$

(where $T^{V}$ denotes as usually the vertical tangent bundle of a fibration). Therefore the holomorphy of $\rho$ is equivalent to the condition

$$
a^{+} \in A^{10}\left(X, \hat{P}^{\rho} \times \text { ad } \hat{\mathfrak{g}}_{+}\right)
$$

(compare with Proposition 5.27). Write

$$
a=\sum_{\lambda \in \operatorname{Spec}[\xi, \cdot]} a_{\lambda}
$$

so that

$$
a^{ \pm}=\sum_{\lambda \in \operatorname{Spec}^{ \pm}[\xi, \cdot]} a_{\lambda},
$$

and take into account that

$$
\begin{gathered}
{[\operatorname{Eig}([\xi, \cdot], \lambda), \operatorname{Eig}([\xi, \cdot], \mu)] \subset \operatorname{Eig}([\xi, \cdot], \lambda+\mu),} \\
\overline{\operatorname{Eig}([\xi, \cdot], \lambda)}=\operatorname{Eig}([\xi, \cdot],-\lambda) .
\end{gathered}
$$

By (17), (18) it follows that

$$
\begin{gathered}
\operatorname{pr}_{\hat{\mathfrak{k}}_{\xi}}[a \wedge a]=\sum_{\lambda \in \operatorname{Spec}^{+}[\xi, \cdot]}\left[a_{\lambda} \wedge a_{-\lambda}\right]+\sum_{\lambda \in \operatorname{Spec}^{-}[\xi, \cdot]}\left[a_{\lambda} \wedge a_{-\lambda}\right]= \\
=-2 \sum_{\lambda \in \operatorname{Spec}^{+}[\xi, \cdot]}\left[a_{\lambda} \wedge \bar{a}_{\lambda}\right]
\end{gathered}
$$


where $[\cdot \wedge \cdot]$ is the (symmetric) bilinear multiplication obtained by multiplying the Lie algebra bracket with the wedge product on 1-forms:

$$
[u \wedge v](x, y):=[u(x), v(y)]-[u(y), v(x)] .
$$

Comparing the curvatures of the two connections $\hat{A}, \hat{A}_{\rho}$, we get

$$
\operatorname{pr}_{\hat{\mathfrak{k}}_{\xi}} F_{\hat{A}}=F_{\hat{A}_{\rho}}+\operatorname{pr}_{\hat{\mathfrak{k}}_{\xi}} \frac{1}{2}[a \wedge a]
$$

But, since $\langle\cdot, \cdot\rangle$ is ad-invariant, we get for any $u, v \in \hat{\mathfrak{g}}$ with $u \in \operatorname{Eig}([\xi, \cdot], \lambda)$

$$
\langle[u, v], \xi\rangle^{\mathbb{C}}=-\langle v,[u, \xi]\rangle^{\mathbb{C}}=\langle[\xi, u], v\rangle^{\mathbb{C}}=\lambda\langle u, v\rangle^{\mathbb{C}} .
$$

Therefore, by the degree formula given in Proposition 2.11, one obtains

$$
\left.\int_{X_{\rho}}\left\langle i \Lambda_{g} F_{\hat{A}}, s(\rho, \xi)\right)\right\rangle v o l_{g}=\frac{2 \pi}{(n-1) !} \operatorname{deg}(\rho, h(\xi))-\sum_{\lambda \in \operatorname{Spec}^{+}[\xi, \cdot]} \lambda\left\|a_{\lambda}\right\|_{L^{2}}^{2},
$$

hence $\frac{2 \pi}{(n-1) !} \operatorname{deg}(\rho, h(\xi)) \geq \int_{X_{\rho}}\left\langle i \Lambda_{g} F_{\hat{A}}, s(\rho, \xi)\right\rangle v_{v o l}$ with equality if and only if $\alpha=0$, i. e. if and only if the connection $\hat{A}$ reduces to a $\hat{K}(\xi)$-connection, which implies that $s(\rho, \xi)$ is an $\hat{A}$-parallel section of $\hat{P} \times$ ad $i$.

Therefore we have shown that $(\hat{A}, \varphi)$ is $\sigma$-semistable, and that, for $\xi \in \mathfrak{i k}$, one has

$$
\frac{2 \pi}{(n-1) !} \operatorname{deg}_{g}(\rho, h(\xi))+\int_{X_{\rho}} \lambda^{\xi}(\varphi, \rho) \text { vol }_{g}=0
$$

if and only if $\rho$ is induced by an $\hat{A}$-parallel $\hat{K}(\xi)$-reduction of $\hat{P}$ and the associated section $s(\rho, \xi) \in A^{0}\left(\hat{P} \times \times_{\text {ad }} i \mathfrak{k}\right)$ belongs to $i \mathfrak{k}_{\hat{A}, \varphi}$, where $\mathfrak{k}_{\hat{A}, \varphi} \subset A^{0}\left(\hat{P} \times{ }_{\text {ad }} \mathfrak{k}\right)$ is the infinitesimal stabilizer algebra of the pair $(\hat{A}, \varphi)$ with respect to the action of the real gauge group $\mathcal{K}:=\Gamma\left(\hat{P} \times_{\mathrm{Ad}} K\right)$. But an $\hat{A}$-parallel $\hat{K}(\xi)$-reduction of $\hat{P}$ defines a holomorphic $Z_{\hat{G}}(\xi)$-reduction of $\hat{\mathcal{Q}}_{J}$. Therefore, the last condition in the definition of polystability is verified.

Using the same method as in the proof of Proposition 1.3 [Te2] one can easily check, using the maximum principle for the operator $P=i \Lambda \bar{\partial} \partial$, that

$$
\mathfrak{g}_{\hat{J}, \varphi}=\mathfrak{k}_{\hat{A}, \varphi} \otimes \mathbb{C}
$$

(compare with Theorem 2.2.1 in [LT]). Taking into account that the elements of $\mathfrak{k}_{\hat{A}, \varphi}$ are $\hat{A}$-parallel sections, we get an $\hat{A}$-parallel reduction of $\hat{P}$ to $Z_{\hat{K}}(\mathfrak{c})$, where $\mathfrak{c} \subset \mathfrak{k}$ is a subalgebra of $\mathfrak{k}$ isomorphic to $\mathfrak{k}_{\hat{A}, \varphi}$. This gives a holomorphic $Z_{\hat{G}}(\mathfrak{c} \otimes \mathbb{C})$-reduction of $\hat{\mathcal{Q}}_{\hat{J}}$, so that $\mathfrak{g}_{\hat{J}, \varphi}$ is a reductive subalgebra of $A^{0}(\hat{Q} \times$ ad $\hat{\mathfrak{g}})$ in the sense of Definition 2.16, which proves the first condition in the definition of polystability. 
Remark 3.4 The proof of the theorem shows that, for a Hermitian-Einstein pair $(\hat{A}, \varphi)$, the following conditions are equivalent:

1. $(\hat{A}, \varphi)$ is irreducible.

2. The associated holomorphic pair $(\hat{J}, \varphi)$ is simple.

\section{Polystable pairs allow Hermitian-Einstein re- ductions}

Definition 4.1 We will denote by $\mathcal{R}(\hat{Q})$ the space of all $\hat{K}$-reductions of $\hat{Q}$ and, for a fixed $K_{0}$-reduction $P_{0}$ of $Q_{0}$, we will denote by $\mathcal{R}_{P_{0}}(\hat{Q})$ the space of all $\hat{K}$-reductions of $\hat{Q}$ which project onto $P_{0}$.

Our final purpose is the following theorem:

Theorem 4.2 Let $(\hat{J}, \varphi)$ be a $\sigma$-polystable pair of type $\left(\hat{Q}, \mathcal{Q}_{0}, \hat{\alpha}\right)$. For any $K_{0}$ reduction $P_{0}$ of $Q_{0}$ there exists a reduction $\hat{P} \in \mathcal{R}_{P_{0}}(\hat{Q})$ which is $\mu$-HermitianEinstein.

In the proof, which will be completed at the and of section 4, we will assume again for simplicity that $g$ is a Gauduchon metric; the statement is true for a general Hermitian metric (see Remark 2.12).

\subsection{The perturbed equation}

Let $(\hat{J}, \varphi)$ be a $\sigma$-polystable pair of type $\left(\hat{Q}, J_{0}\right)$, and let $P_{0}$ be a fixed $K_{0^{-}}$ reduction of $Q_{0}$. We seek a $\hat{K}$-reduction $\hat{P} \subset \hat{Q}$ which projects on $P_{0}$ such that the pair $\left(A_{\hat{P}, \hat{J}}, \varphi\right)$ satisfies the HE equation (see Definition 3.1)), where $A_{\hat{P}, \hat{J}}$ is the Chern connection of the pair $(\hat{P}, \hat{J})$.

By Proposition 2.21 we may suppose that

Assumption: The infinitesimal stabilizer $\mathfrak{g}_{\beta}$ of the polystable pair $\beta=(\hat{J}, \varphi)$ is contained in the centralizer $z(\mathfrak{g}, \hat{G})$.

This assumption has the following important consequence:

Remark 4.3 If this assumption is satisfied, the infinitesimal stabilizer of $\beta$

$$
\mathfrak{k}_{\beta}:=\mathfrak{g}_{\beta} \cap A^{0}\left(\hat{P} \times_{\text {ad }} \mathfrak{k}\right)
$$


with respect to the action of the real gauge group $\mathcal{K}:=\Gamma\left(X, \hat{P} \times{ }_{\mathrm{Ad}} K\right)$ is independent of the $\hat{K}$-reduction $\hat{P}$, and it can be identified with a subalgebra of $z(\mathfrak{k}, \hat{K})$. Moreover, for any $\hat{K}$-reduction $\hat{P}$ of $\hat{Q}$, one has

$$
\mathfrak{g}_{\beta}=\left[\mathfrak{g}_{\beta} \cap A^{0}\left(\hat{P}^{0} \times_{\hat{K}} \mathfrak{k}\right)\right] \otimes \mathbb{C}=\mathfrak{k}_{\beta} \otimes \mathbb{C} .
$$

Indeed, one has

$$
\mathfrak{g}_{\beta} \cap A^{0}\left(\hat{P}^{0} \times_{\hat{K}} \mathfrak{k}\right)=\mathfrak{g}_{\beta} \cap z(\mathfrak{g}, \hat{G}) \cap A^{0}\left(\hat{P} \times_{\hat{K}} \mathfrak{k}\right)=\mathfrak{g}_{\beta} \cap z(\mathfrak{k}, \hat{K}) .
$$

The connected component $G_{\beta}^{0}$ of $e$ in the stabilizer

$$
G_{\beta} \subset \mathcal{G}:=\Gamma\left(\hat{Q} \times{ }_{\mathrm{Ad}} G\right)
$$

of $\beta$ is a connected closed complex subgroup of $\mathcal{G}$ with Lie algebra $\mathfrak{g}_{\beta} \subset z(\mathfrak{g}, \hat{G})$, so it is contained in the connected component $Z(G, \hat{G})^{0}$ of $e$ in the intersection $Z(G, \hat{G}):=G \cap Z(\hat{G})$.

Now it is easy to see that, for every connected closed complex subgroup $H$ of a complex torus $\Theta$, one has $\mathfrak{h}=[\mathfrak{h} \cap \mathfrak{t}] \otimes \mathbb{C}$, where $\mathfrak{t}$ is the Lie algebra of the unique maximal compact subgroup $T$ of $\Theta$.

Therefore, in our case one gets

$$
\mathfrak{g}_{\beta}=\left[\mathfrak{g}_{\beta} \cap z(\mathfrak{k}, \hat{K})\right] \otimes \mathbb{C}=\left[\mathfrak{g}_{\beta} \cap A^{0}\left(\hat{P}^{0} \times_{\hat{K}} \mathfrak{k}\right)\right] \otimes \mathbb{C} .
$$

We fix a $\hat{K}$-reduction $\hat{P} \in \mathcal{R}_{P_{0}}(\hat{Q})$ and seek a solution $\hat{P} \subset \hat{Q}$ of our problem of the form

$$
\hat{P}_{s}=e^{-\frac{s}{2}}(\hat{P}),
$$

where $s \in A^{0}\left(\hat{P} \times_{\text {ad }} i \mathfrak{k}\right)$. Put $h:=e^{s}$. The new (pointwise) moment map $\mu: \Gamma\left(\hat{Q} \times_{\hat{\alpha}} F\right) \rightarrow A^{0}\left(\hat{P}_{s} \times_{\text {ad }} \mathfrak{k}\right)$ associated with the reduction $\hat{P}_{s}$ is given by the formula

$$
\mu_{s}(\varphi)=\operatorname{ad}_{e^{-\frac{s}{2}}}\left(\mu\left(e^{\frac{s}{2}} \varphi\right)\right),
$$

where $\mu$ is the moment map with respect to the initial reduction $\hat{P}$.

Denote by $A^{0}$ the Chern connection of the pair $(\hat{P}, \hat{J})$. The Hermitian-Einstein equation (Definition 3.1) becomes

$$
\operatorname{pr}_{i \mathfrak{k}}\left[i \Lambda_{g}\left(F_{A^{0}}+\bar{\partial}\left(e^{-s} \partial_{0}\left(e^{s}\right)\right)\right)\right]+i \operatorname{ad}_{e^{-\frac{s}{2}}}\left(\mu\left(e^{\frac{s}{2}} \varphi\right)\right)=0,
$$

or, equivalently,

$$
\operatorname{pr}_{i \mathfrak{k}} \operatorname{ad}_{e^{\frac{s}{2}}}\left[i \Lambda_{g}\left(F_{A^{0}}+\bar{\partial}\left(e^{-s} \partial_{0}\left(e^{s}\right)\right)\right)\right]+i\left(\mu\left(e^{\frac{s}{2}} \varphi\right)\right)=0 .
$$


Our perturbed equation is

$$
\operatorname{pr}_{i \mathfrak{k}}\left[i \Lambda_{g}\left(F_{A^{0}}+\bar{\partial}\left(e^{-s} \partial_{0}\left(e^{s}\right)\right)\right]+i \operatorname{ad}_{e^{-\frac{s}{2}}}\left(\mu\left(e^{\frac{s}{2}} \varphi\right)\right)+\varepsilon s=0\right.
$$

which, since $s$ is ad $e_{e^{\frac{s}{2}}}-$ invariant, is equivalent to

$$
\operatorname{ad}_{e^{\frac{s}{2}}} \operatorname{pr}_{i \mathfrak{k}}\left[i \Lambda_{g}\left(F_{A^{0}}+\bar{\partial}\left(e^{-s} \partial_{0}\left(e^{s}\right)\right)\right)\right]+i\left(\mu\left(e^{\frac{s}{2}} \varphi\right)\right)+\varepsilon s=0
$$

Proposition 4.4 There exists a $\hat{K}$-reduction $\hat{P} \in \mathcal{R}_{P_{0}}(\hat{Q})$ such that, denoting by $A^{0}$ the corresponding Chern connection $\hat{A}_{\hat{P}, \hat{J}}$, one has:

A.

$$
\operatorname{pr}_{A^{0}\left(X, i \mathfrak{k}_{\beta}\right)}\left[\operatorname{pr}_{i \mathfrak{k}}\left[i \Lambda_{g} F_{\hat{A}^{0}}\right]+i \mu(\varphi)\right]=0
$$

B. Equation (27) has a solution $\zeta \in A^{0}\left(\hat{P}^{0} \times_{\text {ad }} i \mathfrak{k}_{\beta}^{\perp}\right)$ for $\varepsilon=1$.

\section{Proof:}

Step 1. We start with an arbitrary reduction $\hat{P}^{1} \in \mathcal{R}_{P_{0}}(\hat{Q})$, and we seek a smooth function $v \in \mathcal{C}^{\infty}\left(X, i \mathfrak{k}_{\beta}\right) \subset \mathcal{C}^{\infty}(X, i z(\mathfrak{k}))$ such that the Chern connection $\hat{A}^{2}:=A_{\hat{P}^{2}, \hat{J}}$ associated with the reduction $\hat{P}^{2}:=e^{-\frac{v}{2}}\left(\hat{P}^{1}\right)$ satisfies $(29)$.

By Proposition 5.2, Corollary 5.3 one has

$$
\hat{A}^{2}:=\hat{A}^{1}+\partial v, F_{\hat{A}^{2}}:=F_{\hat{A}^{1}}+\bar{\partial} \partial v .
$$

The new moment map with respect to the reduction $\hat{P}^{2}$ is given by

$$
\mu_{2}(\varphi)=\operatorname{ad}_{e^{-\frac{v}{2}}} \mu_{1}\left(e^{\frac{v}{2}} \varphi\right)=\mu_{1}(\varphi)
$$

because $v$ is central and belongs pointwise to the infinitesimal stabilizer of $\varphi$. Therefore (29) becomes

$$
\operatorname{pr}_{A^{0}\left(X, i \mathfrak{k}_{\beta}\right)}\left[\operatorname{pr}_{i \mathfrak{k}}\left[i \Lambda_{g} F_{\hat{A}^{1}}\right]+i \mu_{1}(\varphi)\right]+i \Lambda \bar{\partial} \partial v=0
$$

On the other hand, applying the polystability condition to central elements of the form $u \in i \mathfrak{k}_{\beta}$ one gets that

$$
\left\langle\operatorname{pr}_{i \mathfrak{k}}\left[i \Lambda_{g} F_{\hat{A}^{1}}\right]+i \mu_{1}(\varphi), u\right\rangle_{L^{2}}=0 \forall u \in i \mathfrak{k}_{\beta}
$$

(compare with Remark 1.12 in the finite dimensional framework). This implies that the Laplace equation (30) has solutions, so we get a reduction $\hat{P}^{2}$ with the desired property.

Step 2. 
Put

$$
\zeta:=-\operatorname{pr}_{i \mathfrak{k}}\left[i \Lambda_{g} F_{\hat{A}^{2}}\right]-i \mu_{2}(\varphi)
$$

and $\hat{P}:=e^{\frac{\zeta}{2}}\left(\hat{P}^{2}\right)$. By Step 1 it follows that $\zeta \in A^{0}\left(\hat{P}^{2} \times\right.$ ad $\left.i \mathfrak{k}_{\beta}^{\perp}\right)$. Since $e^{\frac{\zeta}{2}}$ commutes with $\zeta$, one can also regard $\zeta$ as an element of $A^{0}\left(\hat{P} \times{ }_{\text {ad }} i \mathfrak{k}\right)$ which will be also pointwise orthogonal to $\mathfrak{k}_{\beta}$. By Corollary 5.3 and (24) one has

$$
\begin{gathered}
-\zeta=\operatorname{pr}_{i \mathfrak{k}}\left[i \Lambda_{g}\left(F_{A^{2}}\right]+i\left(\mu_{2}(\varphi)\right)=\right. \\
=\operatorname{pr}_{i \mathfrak{k}}\left[i \Lambda_{g}\left(F_{A^{0}}+\bar{\partial}\left(e^{-\zeta} \partial_{0}\left(e^{\zeta}\right)\right)\right)\right]+i \operatorname{ad}_{e^{-\frac{\zeta}{2}}}\left(\mu_{0}\left(e^{\frac{\zeta}{2}} \varphi\right)\right),
\end{gathered}
$$

which shows that, with these choices, B holds.

\subsection{A priori estimates for the solution $s_{\varepsilon}$}

Lemma 4.5 Fix a $\hat{K}$-reduction $\hat{P}$ as in Proposition 4.4. Let

$$
s \in A^{0}\left(\hat{P} \times{ }_{\text {ad }} i \mathfrak{k}_{\beta}^{\perp}\right)
$$

be a section solving the equation

$$
\operatorname{ad}_{e^{\frac{s}{2}}} \operatorname{pr}_{i \mathfrak{k}}\left[i \Lambda_{g}\left(F_{A^{0}}+\bar{\partial}\left(e^{-s} \partial_{0}\left(e^{s}\right)\right)\right)\right]+i \mu\left(e^{\frac{s}{2}} \varphi\right)+\varepsilon s=0 . \quad\left(e_{\varepsilon}\right)
$$

Put

$$
k^{0}:=\operatorname{pr}_{i \mathfrak{k}}\left[i \Lambda_{g}\left(F_{A^{0}}\right]+i \mu(\varphi)\right.
$$

Then one has:

1. $\frac{1}{2} \cdot P\left(|s|^{2}\right)+\varepsilon \cdot|s|^{2} \leq\left|k^{0}\right| \cdot|s| ;$

2. $m:=\sup _{X}|s| \leq \frac{1}{\varepsilon} \cdot \sup _{X}\left|k^{0}\right|$;

3. $m \leq C \cdot\left(\|s\|_{L^{2}}+\sup _{X}\left|k^{0}\right|\right)^{2}$ where the constant $C$ only depends on $g$ and the fixed $\hat{K}$-reduction $\hat{P}$.

Proof: Taking the pointwise inner product with respect to $\hat{P}$ of both sides of $\left(e_{\varepsilon}\right)$ with $s$, one gets

$$
\left.\left(\operatorname{pr}_{i \mathfrak{k}}\left[i \Lambda_{g} F_{A^{0}}\right], s\right)+\left(i \Lambda_{g} \bar{\partial}\left(e^{-s} \partial_{0}\left(e^{s}\right)\right)\right), s\right)+2 \mu^{-i \frac{s}{2}}\left(e^{\frac{s}{2}} \varphi\right)+\varepsilon|s|^{2}=0 .
$$

On the other hand, note that

$$
\mu^{-i \frac{s}{2}}\left(e^{\frac{s}{2}} \varphi\right)=\mu^{-i \frac{s}{2}}(\varphi)+\mathfrak{e}^{\frac{s}{2}}(\varphi),
$$

where $\mathfrak{e}^{s}(\varphi)$ is the $\mathbb{R}_{\geq 0}$-valued function which maps every $x \in X$ to the energy of the curve $[0,1] \ni t \mapsto e^{\frac{t s}{2}} \varphi(x) \in F_{x}$. 
Therefore,

$$
\begin{array}{r}
\left.\left(i \Lambda_{g} \bar{\partial}\left(e^{-s} \partial_{0}\left(e^{s}\right)\right)\right), s\right)+\varepsilon|s|^{2}=-\left(\operatorname{pr}_{i \mathfrak{k}}\left[i \Lambda_{g}\left(F_{A^{0}}\right], s\right)-2 \mu^{-i \frac{s}{2}}\left(e^{\frac{s}{2}} \varphi\right) \leq\right. \\
-\left(\operatorname{pr}_{i \mathfrak{k}}\left[i \Lambda_{g} F_{A^{0}}\right], s\right)-2 \mu^{-i \frac{s}{2}}(\varphi)=-\left(\operatorname{pr}_{i \mathfrak{k}}\left[i \Lambda_{g} F_{A^{0}}\right]+i \mu(\varphi), s\right)=-\left(k^{0}, s\right) .
\end{array}
$$

We introduce now the real analytic function $\eta: \mathbb{R} \rightarrow \mathbb{R}_{>0}$ defined by

$$
\eta(t)=\left\{\begin{array}{ccc}
\sqrt{\frac{1-e^{-t}}{t}} & \text { if } & t \neq 0 \\
1 & \text { if } & t=0
\end{array}\right.
$$

(see section 5.4). We use the formalism explained in section 5.4. By Proposition 5.16 the inequality (33) becomes

$$
\frac{1}{2} P\left(|s|^{2}\right)+\left|\eta([s, \cdot])\left(\partial_{0}(s)\right)\right|^{2}+\varepsilon|s|^{2} \leq-\left(k^{0}, s\right) .
$$

This proves the claim.

2. follows from 1. by the Maximum Principle.

3. From 1. we deduce

$$
P\left(|s|^{2}\right) \leq 2 \cdot\left|k^{0}\right||s| \leq|s|^{2}+\left|k^{0}\right|^{2} \leq|s|^{2}+\sup _{X}\left|k^{0}\right|^{2},
$$

so, since $\sup _{X}\left|k^{0}\right|^{2}$ is a constant, Lemma 3.3.2 [LT] implies

$$
\begin{gathered}
m \leq C \cdot\left(\left\||s|^{2}\right\|_{L^{1}}+\sup _{X}\left|k^{0}\right|^{2}\right)=C \cdot\left(\|s\|_{L^{2}}^{2}+\sup _{X}\left|s^{0}\right|^{2}\right) \\
\leq C \cdot\left(\|s\|_{L^{2}}+\sup _{X}\left|k^{0}\right|\right)^{2} .
\end{gathered}
$$

\subsection{Solving the equation $\left(e_{\varepsilon}\right)$ for $\varepsilon \in(0,1]$.}

Let

$$
l(\varepsilon, s):=\operatorname{ad}_{e^{\frac{s}{2}}} \operatorname{pr}_{i \mathfrak{k}}\left[i \Lambda_{g}\left(F_{A^{0}}+\bar{\partial}\left(e^{-s} \partial_{0}\left(e^{s}\right)\right)\right)\right]+i \mu\left(e^{\frac{s}{2}} \varphi\right)+\varepsilon s
$$

be the left hand term of the equation $\left(e_{\varepsilon}\right)$.

We state first the following regularity result: Choose $p \in \mathbb{N}$ such that the Sobolev space $L_{k}^{p}$ is an algebra for $k \geq 1$.

Lemma 4.6 Let $s \in L_{2}^{p}\left(\hat{P} \times{ }_{\text {ad }} i \mathfrak{k}_{\beta}^{\perp}\right)$ such that $l(\varepsilon, s)=0$ for some $\varepsilon \in \mathbb{R}$. Then $s \in \mathcal{C}^{\infty}\left(\hat{P} \times\right.$ ad $\left.i \mathfrak{k}_{\beta}^{\perp}\right)$. 
Proof: Recall first that, by Proposition 5.4, one has

$$
\operatorname{ad}_{e^{\frac{s}{2}}}\left(F_{A^{0}}+\bar{\partial}\left(e^{-s} \partial_{0}\left(e^{s}\right)\right)\right)=F_{A_{s}},
$$

where $A_{s}:=e^{-\frac{s}{2}}\left(A^{0}+e^{-s} \partial_{0}\left(e^{s}\right)\right) \in \mathcal{A}(\hat{P})$. The components of the linear connection defined by $A_{s}$ on the bundle $\hat{P} \times$ ad $\mathfrak{g}$ are

$$
\bar{\partial}_{A_{s}}=\operatorname{ad}_{e^{\frac{s}{2}}} \circ \bar{\partial}_{A^{0}} \circ \operatorname{ad}_{e^{-\frac{s}{2}}}, \partial_{A_{s}}=\operatorname{ad}_{e^{-\frac{s}{2}}} \circ \partial_{A^{0}} \circ \operatorname{ad}_{e^{\frac{s}{2}}},
$$

which can be rewritten as

$$
\bar{\partial}_{A_{s}}=\bar{\partial}-\bar{\partial}\left(e^{\frac{s}{2}}\right) e^{-\frac{s}{2}}, \partial_{A_{s}}=\partial_{0}+e^{-\frac{s}{2}} \partial_{0}\left(e^{\frac{s}{2}}\right) .
$$

Here the forms $\bar{\partial}\left(e^{\frac{s}{2}}\right) e^{-\frac{s}{2}}, e^{-\frac{s}{2}} \partial_{0}\left(e^{\frac{s}{2}}\right)$ operate on $\hat{P} \times$ ad $\mathfrak{g}$ via the adjoint representation $a \mapsto[a, \cdot]$. On the other hand, by formulae (74), (75) in the Appendix, these forms can be expressed in terms of $\bar{\partial} s, \partial_{0} s$ as

$$
\bar{\partial}\left(e^{\frac{s}{2}}\right) e^{-\frac{s}{2}}=\frac{1}{2} \psi\left(-\left[\frac{s}{2}, \cdot\right]\right)(\bar{\partial} s), e^{-\frac{s}{2}} \partial_{0}\left(e^{\frac{s}{2}}\right)=\frac{1}{2} \psi\left(\left[\frac{s}{2}, \cdot\right]\right)\left(\partial_{0} s\right),
$$

where $\psi$ is the positive real analytic function

$$
\psi(t)=\left\{\begin{array}{ccc}
\frac{e^{t}-1}{t} & \text { if } \quad t \neq 0 \\
1 & \text { if } \quad t=0 .
\end{array}\right.
$$

Let $\Psi: \hat{P} \times$ ad $i \mathfrak{k} \rightarrow \operatorname{Herm}(\hat{P} \times$ ad $\mathfrak{g})$ be the (non-linear!) real analytic bundle map defined by

$$
a \mapsto \frac{1}{2} \psi\left(\left[\frac{a}{2}, \cdot\right]\right) .
$$

We get

$$
\bar{\partial}_{A_{s}}=\bar{\partial}-\Psi(-s)(\bar{\partial} s), \partial_{A_{s}}=\partial_{0}+\Psi(s)\left(\partial_{0} s\right) .
$$

This gives for the $(1,1)$ curvature form of $A_{s}$

$$
\begin{gathered}
F_{A_{s}}=F_{A^{0}}+d_{A^{0}}\left(\Psi(s)\left(\partial_{0} s\right)-\Psi(-s)(\bar{\partial} s)\right)+ \\
+\left(\Psi(s)\left(\partial_{0} s\right)-\Psi(-s)(\bar{\partial} s)\right) \wedge\left(\Psi(s)\left(\partial_{0} s\right)-\Psi(-s)(\bar{\partial} s)\right)= \\
=2 \Psi(s)\left(\bar{\partial} \partial_{0} s\right)+(1-\Psi(-s))\left(F_{A^{0}}\right)+\bar{\partial}(\Psi(s)) \wedge \partial_{0} s-\partial_{0}(\Psi(-s)) \wedge \bar{\partial} s \\
-\Psi(s)\left(\partial_{0} s\right) \wedge \Psi(-s)(\bar{\partial} s)-\Psi(-s)(\bar{\partial} s) \wedge \Psi(s)\left(\partial_{0} s\right) .
\end{gathered}
$$

Therefore the equation $\left(e_{\varepsilon}\right)$ is equivalent to an equation of the form

$$
i \Lambda \bar{\partial} \partial_{0} s=\mathcal{F}\left(\varepsilon, s, d_{A^{0}}(s)\right) .
$$

The left composition lemma applies on the right, more precisely, $\mathcal{F}\left(\varepsilon, s, d_{A^{0}}(s)\right)$ belongs to $L_{k-1}^{p}$ when $s$ belongs to $L_{k}^{p}$. The result follows by standard bootstrapping.

The left composition lemma gives also the following 
Remark 4.7 The map $l$ extends to a differentiable map

$$
l: \mathbb{R} \times L_{2}^{p}\left(\hat{P} \times \text { ad } i \mathfrak{k}_{\beta}^{\perp}\right) \rightarrow L^{p}\left(\hat{P} \times{ }_{\text {ad }} i \mathfrak{k}_{\beta}^{\perp}\right) .
$$

Lemma 4.8 Suppose that $l(\varepsilon, s)=0$ with $s \in A^{0}\left(\hat{P} \times{ }_{\text {ad }} i \mathfrak{k}_{\beta}^{\perp}\right)$ and $\varepsilon>0$. Then the partial derivative

$$
\frac{\partial}{\partial s} l(e, s): L_{2}^{p}\left(\hat{P} \times{ }_{\text {ad }} i \mathfrak{k}_{\beta}^{\perp}\right) \rightarrow L^{p}\left(\hat{P} \times \text { ad } i \mathfrak{k}_{\beta}^{\perp}\right)
$$

is an invertible operator.

\section{Proof:}

For $\dot{s} \in L_{2}^{p}\left(\hat{P} \times\right.$ ad $\left.i \mathfrak{k}_{\beta}^{\perp}\right)$, put

$$
u:=\left(d_{\frac{s}{2}} \exp \right)\left(\frac{\dot{s}}{2}\right) e^{-\frac{s}{2}} \in \hat{P} \times_{\text {ad }}\left(i \mathfrak{k}_{\beta}^{\perp} \otimes \mathbb{C}\right) .
$$

The components of $u$ in $\hat{P} \times$ ad $i \mathfrak{k}_{\beta}^{\perp}$ and $\hat{P} \times$ ad $\mathfrak{k}_{\beta}^{\perp}$ are respectively

$$
u_{h}=\frac{1}{2}\left(u+\operatorname{ad}_{e^{-\frac{s}{2}}} u\right), u_{a}=\frac{1}{2}\left(u-\operatorname{ad}_{e^{-\frac{s}{2}}} u\right) .
$$

One has

$$
\begin{gathered}
\left(\frac{\partial}{\partial s} F_{A_{s}}\right)(\dot{s})=d_{A_{s}}\left(\frac{\partial}{\partial s} A_{s}\right)=d_{A_{s}}\left(\partial_{A_{s}}\left(\operatorname{ad}_{e^{-\frac{s}{2}}} u\right)-\bar{\partial}_{A_{s}} u\right)= \\
-\partial_{A_{s}} \bar{\partial}_{A_{s}} u+\bar{\partial}_{A_{s}} \partial_{A_{s}}\left(\operatorname{ad}_{e^{-\frac{s}{2}}} u\right)=\left[u_{a}, F_{A_{s}}\right]+\left(\bar{\partial}_{A_{s}} \partial_{A_{s}}-\partial_{A_{s}} \bar{\partial}_{A_{s}}\right)\left(u_{h}\right) .
\end{gathered}
$$

On the other hand, the same computation as in the finite dimensional framework (see the proof of Proposition 1.15) give

$$
\begin{aligned}
\frac{\partial}{\partial s} i \mu\left(e^{\frac{s}{2}} \varphi\right) & =d(i \mu)\left(u_{e^{\frac{s}{2}} \varphi}^{\#}\right)=d(i \mu)\left(\left[u_{h}^{\#}\right]_{e^{\frac{s}{2}} \varphi}+\left[u_{a}^{\#}\right]_{e^{\frac{s}{2}} \varphi}\right)= \\
& =d(i \mu)\left(\left[u_{h}^{\#}\right]_{e^{\frac{s}{2}} \varphi}\right)+\left[u_{a}, i \mu\left(e^{\frac{s}{2}} \varphi\right)\right] .
\end{aligned}
$$

Therefore

$$
\begin{gathered}
\frac{\partial}{\partial s} l(\varepsilon, s)(\dot{s})= \\
i \Lambda_{g}\left(\bar{\partial}_{A_{s}} \partial_{A_{s}}-\partial_{A_{s}} \bar{\partial}_{A_{s}}\right)\left(u_{h}\right)+d(i \mu)\left(\left[u_{h}^{\#}\right]_{e^{\frac{s}{2}} \varphi}\right)+\left[u_{a}, l(\varepsilon, s)-\varepsilon s\right]+\varepsilon \dot{s} .
\end{gathered}
$$

Recall the following well-known identity which holds for any unitary integrable connection $B$ on a Hermitian vector bundle:

$$
P\left(|v|^{2}\right):=i \Lambda_{g} \bar{\partial} \partial|v|^{2}=\left(i \Lambda_{g} \bar{\partial}_{B} \partial_{B} v, v\right)-\left(v, i \Lambda_{g} \partial_{B} \bar{\partial}_{B} v\right)-\left|d_{B}(v)\right|^{2} .
$$


In our case, using the Euclidean inner product on $\hat{P} \times_{\text {ad }} i \mathfrak{k}$, this yields

$$
P\left|u_{h}\right|^{2}=\left(i \Lambda_{g}\left(\bar{\partial}_{A_{s}} \partial_{A_{s}}-\partial_{A_{s}} \bar{\partial}_{A_{s}}\right) u_{h}, u_{h}\right)-\left|d_{A_{s}}\left(u_{h}\right)\right|^{2} .
$$

Therefore, for a pair $(\varepsilon, s)$ with $l(\varepsilon, s)=0$, and using the well known formulae obtained in the finite dimensional case, we get

$$
\begin{gathered}
\left\langle\frac{\partial}{\partial s} l(\varepsilon, s)(\dot{s}), u_{h}\right\rangle_{L^{2}}=\left\|d_{A_{s}} u_{h}\right\|_{L^{2}}^{2}+\left\|\left[u_{h}^{\#}\right]_{e^{\frac{s}{2}} \varphi}\right\|^{2}-\varepsilon\left\langle\left[u_{a}, s\right], u_{h}\right\rangle+2 \varepsilon\left\langle\frac{\dot{s}}{2}, u_{h}\right\rangle \\
\geq \frac{1}{2} \varepsilon\|\dot{s}\|_{L^{2}}^{2}
\end{gathered}
$$

by Proposition 5.10 in the Appendix. This proves that $\frac{\partial}{\partial s} l(\varepsilon, s)$ is an injective operator. It suffices to notice that its index vanishes.

Lemma 4.9 There is a positive constant $C=C\left(A^{0}, \varphi\right)$ such that the following estimate holds:

$$
C\|v\|_{L^{2}}^{2} \leq\left\|\bar{\partial}_{A^{0}} v\right\|_{L^{2}}^{2}+\left\|v_{\varphi}^{\#}\right\|_{L^{2}}^{2} \forall v \in A^{0}\left(\hat{P} \times_{\text {ad }}\left(i \mathfrak{k}_{\beta}^{\perp} \otimes \mathbb{C}\right)\right) .
$$

Proof: If not, there would exist a sequence $\left(v_{n}\right)_{n}$ with $\left\|v_{n}\right\|_{L^{2}}^{2} \equiv 1$ such that

$$
\left\|\bar{\partial}_{A^{0}} v_{n}\right\|_{L^{2}}^{2}+\left\|\left(v_{n}\right)_{\varphi}^{\#}\right\|_{L^{2}}^{2} \rightarrow 0
$$

But this implies that $v_{n}$ has a subsequence which is weakly convergent in $L_{1}^{2}$ and strongly convergent in $L^{2}$. The limit, say $v_{0}$, satisfies $\bar{\partial}_{A^{0}}\left(v_{0}\right)=0$, hence it is smooth. Noting that $(\cdot)_{\varphi}^{\#}$ can be regarded as a smooth linear bundle morphism

$$
\left.\hat{P} \times \text { ad }\left(i \mathfrak{k}_{\beta}^{\perp} \otimes \mathbb{C}\right)\right) \longrightarrow \varphi^{*}\left(T^{\mathrm{vert}}(E)\right),
$$

one gets

$$
\bar{\partial}_{A^{0}} v_{0}=0,\left(v_{0}\right)_{\varphi}^{\#}=0 .
$$

Therefore, by Remark 4.3, one has $v_{0} \in \mathfrak{g}_{\beta}=\mathfrak{k}_{\beta} \otimes \mathbb{C}$. But this contradicts $v_{0} \in A^{0}\left(\hat{P} \times_{\text {ad }}\left(i \mathfrak{k}_{\beta}^{\perp} \otimes \mathbb{C}\right)\right)$.

Note that this lemma holds for any holomorphic pair $\beta$ and $\hat{K}$-reduction $\hat{P}$ such that the relation $\mathfrak{g}_{\beta}=\mathfrak{k}_{\beta} \otimes \mathbb{C}$ between its infinitesimal stabilizers holds.

For any $s \in A^{0}\left(\hat{P} \times\right.$ ad $\left.\left(i \mathfrak{k}_{\beta}^{\perp}\right)\right)$ put

$$
m_{s}:=\sup _{X}|s| .
$$


Corollary 4.10 There exists a positive constant $C\left(A^{0}, \varphi, m_{s}\right)$ such that for all $s \in A^{0}\left(\hat{P} \times \times_{\text {ad }}\left(i \mathfrak{k} \frac{\perp}{\beta}\right)\right)$ and $w \in A^{0}\left(\hat{P} \times{ }_{\text {ad }}\left(i \mathfrak{k}_{\beta}^{\perp} \otimes \mathbb{C}\right)\right)$ it holds

$$
C\left(A^{0}, \varphi, m_{s}\right)\|w\|_{L^{2}}^{2} \leq\left\|\bar{\partial}_{A_{s}} w\right\|_{L^{2}}^{2}+\left\|[w]_{e^{\frac{s}{2} \varphi}}^{\#}\right\|^{2} .
$$

Proof: Defining $v:=\operatorname{ad}_{e^{-\frac{s}{2}}} w$ one has

$$
\bar{\partial}_{A_{s}} w=\operatorname{ad}_{e^{\frac{s}{2}}}\left(\bar{\partial}_{A^{0}} v\right),[w]_{e^{\frac{s}{2}} \varphi}^{\#}=\left(e^{\frac{s}{2}}\right)_{*, \varphi}\left(v_{\varphi}^{\#}\right) .
$$

But one has estimates of the form

$$
\begin{gathered}
\left\|\operatorname{ad}_{e^{\frac{s}{2}}}\left(\bar{\partial}_{A^{0}} v\right)\right\|_{L^{2}}^{2} \geq C^{\prime}\left(m_{s}\right)\left\|\left(\bar{\partial}_{A^{0}} v\right)\right\|_{L^{2}}^{2},\left\|\left(e^{\frac{s}{2}}\right)_{*, \varphi}\left(v_{\varphi}^{\#}\right)\right\|_{L^{2}}^{2} \geq C^{\prime \prime}\left(\varphi, m_{s}\right)\left\|\left(v_{\varphi}^{\#}\right)\right\|_{L^{2}}^{2} \\
\|v\|_{L^{2}}^{2} \geq C^{\prime \prime \prime}\left(m_{s}\right)\|w\|_{L^{2}}^{2}
\end{gathered}
$$

It suffices to apply the previous lemma.

Let $\left(s_{\varepsilon}\right)_{\varepsilon}$ be a smooth family in $A^{0}\left(\hat{P} \times\right.$ ad $\left.i \mathfrak{k}_{\beta}^{\perp}\right)$ such that for all $\varepsilon$ it holds $l\left(\varepsilon, s_{\varepsilon}\right)=0$. Put

$$
u=\left(\frac{d}{d \varepsilon} \exp \left(\frac{s}{2}\right)\right) e^{-\frac{s}{2}}, \dot{s}:=\frac{d}{d \varepsilon} s .
$$

Lemma 4.11 One has an estimate of the form

$$
\sup _{X}\left|u_{h}\right| \leq C\left(m_{s}\right)
$$

Proof: By (40) one gets

$$
\begin{gathered}
0=\frac{\partial}{\partial \varepsilon} l\left(\varepsilon, s_{\varepsilon}\right)= \\
i \Lambda_{g}\left(\bar{\partial}_{A_{s}} \partial_{A_{s}}-\partial_{A_{s}} \bar{\partial}_{A_{s}}\right)\left(u_{h}\right)+d(i \mu)\left(\left[u_{h}^{\#}\right]_{e^{\frac{s}{2}} \varphi}\right)-\varepsilon\left[u_{a}, s\right]+\varepsilon \dot{s}+s .
\end{gathered}
$$

Taking this time inner product with $u_{h}$ pointwise and using again (41) and the inequalities in Proposition 5.10, one gets

$$
\begin{gathered}
0=P\left(\left|u_{h}\right|^{2}\right)+\left|d_{A_{s}} u_{h}\right|^{2}+\left|\left[u_{h}^{\#}\right]_{e^{\frac{s}{2}} \varphi}\right|^{2}-\varepsilon\left(\left[u_{a}, s\right], u_{h}\right)+\varepsilon\left(\dot{s}, u_{h}\right)\left(\dot{s}, u_{h}\right)+\left(s, u_{h}\right) \geq \\
P\left(\left|u_{h}\right|^{2}\right)+\left|d_{A_{s}} u_{h}\right|^{2}+\left|\left[u_{h}^{\#}\right]_{e^{\frac{s}{2}} \varphi}\right|^{2}+\left(s, u_{h}\right)
\end{gathered}
$$

Integrating over $X$ one gets

$$
\left\|d_{A_{s}} u_{h}\right\|_{L^{2}}^{2}+\left\|\left[u_{h}^{\#}\right]_{e^{\frac{s}{2}}}\right\|_{L^{2}}^{2} \leq\|s\|_{L^{2}}\left\|u_{h}\right\|_{L^{2}} \leq \sqrt{\operatorname{Vol}(X)} m_{s}\left\|u_{h}\right\|_{L^{2}}
$$


whereas the term on the left is larger than $C\left(A^{0}, \varphi, m_{s}\right)\left\|u_{h}\right\|_{L^{2}}^{2}$ by Corollary 4.10. This gives an $L^{2}$-estimate of the form

$$
\left\|u_{h}\right\|_{L^{2}} \leq C_{1}\left(A_{0}, \varphi, m_{s}\right) .
$$

Coming back to (45) we see that

$$
P\left(\left|u_{h}\right|^{2}\right) \leq-\left(s, u_{h}\right) \leq|s|\left|u_{h}\right| \leq \frac{1}{2}\left(\left|u_{h}\right|^{2}+m_{s}^{2}\right) .
$$

Using now Lemma 3.3.2 in [LT] and (46), one gets an estimate of the form

$$
\sup _{X}\left(\left|u_{h}\right|^{2}\right) \leq C(g)\left(\left\|u_{h}\right\|_{L^{2}}^{2}+\frac{1}{2} m_{s}^{2}\right) \leq C\left(g, A^{0}, \varphi, m_{s}\right) .
$$

Using again the formulae (38) we get

$$
\begin{gathered}
\bar{\partial}_{A_{s}} \partial_{A_{s}}-\partial_{A_{s}} \bar{\partial}_{A_{s}}=\bar{\partial} \partial_{0}-\partial_{0} \bar{\partial} \\
-\Psi(-s)(\bar{\partial} s) \wedge \partial_{0}-\Psi(-s)(\bar{\partial} s) \wedge \Psi(s)\left(\partial_{0} s\right)-\Psi(s)\left(\partial_{0} s\right) \wedge \bar{\partial}+\Psi(s)\left(\partial_{0} s\right) \wedge \Psi(-s)(\bar{\partial} s) \\
+\Psi(s)\left(\bar{\partial} \partial_{0} s\right)+\Psi(-s)\left(\partial_{0} \bar{\partial} s\right)+\partial_{0}(\Psi(-s)) \wedge \bar{\partial} s+\bar{\partial}(\Psi(s)) \wedge \partial_{0} s
\end{gathered}
$$

We plug the identity (48) in (44) and use standard elliptic $L^{p}$ - estimates for the operator $i \Lambda_{g}\left(\bar{\partial} \partial_{0}-\partial_{0} \bar{\partial}\right)$.

Note first that, since $\Psi$ is of class $\mathcal{C}^{1}$, one has an estimate of the form

$$
\left|d_{A^{0}}(\Psi(s))\right| \leq C\left(m_{s}\right)\left|d_{A^{0}} s\right| .
$$

Recalling that, by (43), $\sup _{X}\left|u_{h}\right|$ is bounded by a constant $C\left(m_{s}\right)$, we obtain

$$
\begin{aligned}
\left\|u_{h}\right\|_{L_{2}^{p}} & \leq c\left(m_{s}\right)\left(1+\|s\|_{L_{1}^{2 p}}\left\|u_{h}\right\|_{L_{1}^{2 p}}+\|s\|_{L_{1}^{2 p}}^{2}+\|s\|_{L_{2}^{p}}\right)+ \\
& +\left\|d(i \mu)\left(\left[u_{h}^{\#}\right]_{e^{\frac{s}{2}} \varphi}\right)-\varepsilon\left[u_{a}, s\right]+\varepsilon \dot{s}+s\right\|_{L^{p}}
\end{aligned}
$$

On the other hand, by the formulae obtained in the proof of Proposition 5.10, one can express $\dot{s}$ in terms of $u_{h}$ as

$$
\dot{s}=2 \theta\left(\left[\frac{s}{2}, \cdot\right]\right)\left(u_{h}\right)
$$

where this time $\theta(t)=\frac{2 t}{e^{t}-e^{-t}}$. Since the bundle map $\hat{P} \times$ ad $i \mathfrak{k} \rightarrow \operatorname{Herm}(\hat{P} \times$ ad $\mathfrak{g})$ given by $a \mapsto 2 \theta\left(\left[\frac{a}{2}, \cdot\right]\right)$ is of class $\mathcal{C}^{2}$, one gets estimates of the form

$$
|\dot{s}| \leq c\left(m_{s}\right)\left|u_{h}\right|,\|\dot{s}\|_{L_{2}^{p}} \leq c\left(m_{s}\right)\left\|u_{h}\right\|_{L_{2}^{p}},
$$


in particular, from (43) we deduce

$$
\sup _{X}|\dot{s}| \leq c\left(m_{s}\right) .
$$

Expressing $u_{h}$ and $u_{a}$ similarly in terms of $\dot{s}$, one gets

$$
\left|u_{a}\right| \leq c\left(m_{s}\right)|\dot{s}|,\left\|u_{h}\right\|_{L_{1}^{2 p}} \leq c\left(m_{s}\right)\|\dot{s}\|_{L_{1}^{2 p}} .
$$

Note finally that (since $u_{h}$ and $s$ are bounded in terms of $\left.m_{s}\right)\left[u_{h}^{\#}\right]_{e^{\frac{s}{2}} \varphi}$ remains in a compact subset $K\left(\varphi, m_{s}\right)$ of the vertical tangent bundle $T^{\text {vert }}(E)$. Therefore, one gets

$$
\sup _{X}\left|d(i \mu)\left(\left[u_{h}^{\#}\right]_{e^{\frac{s}{2}} \varphi}\right)\right| \leq c\left(m_{s}\right) .
$$

Summarizing, we obtain an estimate of the form

$$
\|\dot{s}\|_{L_{2}^{p}} \leq c\left(m_{s}\right)\left(1+\|s\|_{L_{1}^{2 p}}\|\dot{s}\|_{L_{1}^{2 p}}+\|s\|_{L_{1}^{2 p}}^{2}+\|s\|_{L_{2}^{p}}\right) .
$$

Using the general inequality

$$
\|x\|_{L_{1}^{2 p}} \leq C \cdot\left(\sup _{X}|x|^{\frac{1}{2}}\right) \cdot\|x\|_{L_{2}^{p}}^{\frac{1}{2}}+\|x\|_{L^{2 p}},
$$

([Au] Theorem 3.69; the constant $C$ depends only on $p$ and the dimension of $X)$ and (50) one proceeds as in the proof of [LT] Proposition 3.3.5 to obtain an inequality of the form

$$
\|\dot{s}\|_{L_{2}^{p}} \leq c\left(m_{s}\right)\left(1+\|s\|_{L_{2}^{p}}\right) .
$$

Integrating from $\varepsilon$ to 1 this yields (compare with [LT], Proposition 3.3.5)

Proposition 4.12 Let $\left(s_{\varepsilon}\right)_{\varepsilon \in\left(\varepsilon_{0}, 1\right]}$ be a smooth family in $A^{0}\left(\hat{P} \times_{\text {ad }} i \mathfrak{k}_{\beta}^{\perp}\right)$ such that for all $\varepsilon$ it holds $l\left(\varepsilon, s_{\varepsilon}\right)=0$. Then one has an estimate of the form

$$
\left\|s_{\varepsilon}\right\|_{L_{2}^{p}} \leq e^{c\left(m_{s_{\varepsilon}}\right)(1-\varepsilon)}\left(1+\left\|s_{1}\right\|_{L_{2}^{p}}\right) \forall \varepsilon \in\left(\varepsilon_{0}, 1\right] .
$$

We know that the equation $l(\cdot, 1)=0$ has a smooth solution $\zeta$ (Proposition 4.4). Consider the set

$$
\mathcal{S}:=\left\{\mathfrak{s}:\left(\varepsilon_{\mathfrak{s}}, 1\right] \rightarrow A^{0}\left(\hat{P} \times_{\text {ad }} i \mathfrak{k}_{\beta}^{\perp}\right) \mid 0 \leq \varepsilon_{\mathfrak{s}}<1, l(\varepsilon, \mathfrak{s}(\varepsilon)) \equiv 0, \mathfrak{s}(1)=\zeta\right\} .
$$

$\mathcal{S}$ is nonempty by Lemma 4.8, implicit function theorem and Lemma 4.6. Writing $\mathfrak{s}_{1} \leq \mathfrak{s}_{2}$ when $\varepsilon_{\mathfrak{s}_{2}} \leq \varepsilon_{\mathfrak{s}_{1}}$ and $\mathfrak{s}_{1}=\left.\mathfrak{s}_{2}\right|_{\left(\varepsilon_{\mathfrak{s}_{1}}, 1\right]}, \mathcal{S}$ becomes an inductively ordered set, hence there is a maximal element in $\mathcal{S}$. 


\section{Proposition 4.13}

1. Let $\mathfrak{s}:\left(\varepsilon_{\mathfrak{s}}, 1\right] \rightarrow A^{0}\left(\hat{P} \times{ }_{\mathrm{ad}} i \mathfrak{k}_{\beta}^{\perp}\right) \in \mathcal{S}$ and put $s_{\varepsilon}:=\mathfrak{s}(\varepsilon)$. If

$$
\sup \left\{\left\|s_{\varepsilon}\right\|_{L^{2}} \mid \varepsilon \in\left(\varepsilon_{\mathfrak{s}}, 1\right]\right\}<\infty,
$$

then the strong limit $\lim _{\varepsilon \rightarrow \varepsilon_{\mathfrak{s}}} s_{\varepsilon}$ exists in $L_{2}^{p}$, and is a smooth solution of the equation $l\left(\varepsilon_{\mathfrak{s}}, \cdot\right)=0$.

2. For any maximal element $\mathfrak{s} \in \mathcal{S}$, one has $\varepsilon_{\mathfrak{s}}=0$.

\section{Proof:}

1. By assumption and the third statement in Lemma 4.5 one gets an uniform bound

$$
m_{s_{\varepsilon}}=\sup _{X}\left|s_{\varepsilon}\right| \leq M, \forall \varepsilon \in\left(\varepsilon_{\mathfrak{s}}, 1\right]
$$

Therefore, the family $\left(s_{\varepsilon}\right)_{\varepsilon \in\left(\varepsilon_{\mathfrak{s}}, 1\right]}$ is bounded in $L_{2}^{p}$ by Proposition 4.12 . Using now the inequality (53), we see that $\frac{d}{d \varepsilon} s_{\varepsilon}$ is also bounded in $L_{2}^{p}$, hence the map $\mathfrak{s}:\left(\varepsilon_{\mathfrak{s}}, 1\right] \rightarrow L_{2}^{p}\left(\hat{P} \times\right.$ ad $\left.i \mathfrak{k}_{\beta}^{\perp}\right)$ is Lipschitz. Therefore one has a strong limit

$$
s=\lim _{\varepsilon \rightarrow \varepsilon_{\mathfrak{s}}} s_{\varepsilon}
$$

which will be a solution of the equation $l\left(\varepsilon_{\mathfrak{s}}, \cdot\right)=0$ by Remark 4.7 , and will be smooth by Lemma 4.6.

2. If $\varepsilon_{\mathfrak{s}}>0$, the second statement of Lemma 4.5 gives an uniform bound of the form (55). Therefore, the first part of this proposition applies and gives a strong $L_{2}^{p}-\operatorname{limit} s=\lim _{\varepsilon \rightarrow \varepsilon_{\mathfrak{s}}} s_{\varepsilon}$ which is smooth and solves the equation $l\left(\varepsilon_{\mathfrak{s}}, \cdot\right)=0$.

Using Lemma 4.8 and the implicit function theorem we get a smooth extension of $\mathfrak{s}$ on a larger interval, contradicting maximality.

This gives immediately the following crucial result:

\section{Theorem 4.14}

1. There exists a smooth family $\left(s_{\varepsilon}\right)_{\varepsilon \in(0,1]}$ with $l\left(\varepsilon, s_{\varepsilon}\right) \equiv 0$.

2. If $\sup \left\{\left\|s_{\varepsilon}\right\|_{L^{2}} \mid \varepsilon \in(0,1]\right\}<\infty$, then $s_{\varepsilon}$ converges strongly in $L_{2}^{p}$ to a smooth solution $s$ of the equation (25) as $\varepsilon \rightarrow 0$.

Therefore, when $\sup \left\{\left\|s_{\varepsilon}\right\|_{L^{2}} \mid \varepsilon \in(0,1]\right\}<\infty$, one gets a Hermitian-Einstein reduction $\hat{P}_{s}:=e^{-\frac{s}{2}}(\hat{P}) \in \mathcal{R}_{P_{0}}(\hat{Q})$. 


\subsection{Destabilizing the pair in the unbounded case}

\subsubsection{Estimates in the unbounded case}

Let $\left(s_{\varepsilon}\right)_{\varepsilon \in(0,1]}$ be the smooth family given by Theorem 4.14. Our first aim is to get a uniform $L_{1}^{2}$ - bound for the $L^{2}$-normed section

$$
\sigma_{\varepsilon}:=\frac{s_{\varepsilon}}{\left\|s_{\varepsilon}\right\|_{L^{2}}} .
$$

By the third statement of Lemma 4.5, we get uniform bounds

$$
\sup _{X}\left|\sigma_{\varepsilon}\right| \leq M, \sup _{x \in X}\left\{|\lambda| \mid \lambda \in \operatorname{Spec}\left(\left[\left(\sigma_{\varepsilon}\right)_{x}, \cdot\right]\right)\right\} \leq M
$$

for all $\varepsilon>0$ for which $\left\|s_{\varepsilon}\right\|_{L^{2}} \geq 1$.

Remark 4.15 Fix $\alpha \in(0,1)$. Then, for any $u \in \mathbb{R}, n>0$ one has

1.

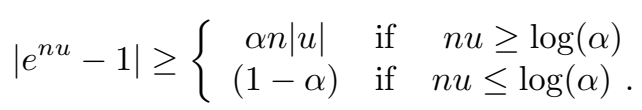

2. Supposing $|u| \leq M$, we get

$$
\left|e^{n u}-1\right| \geq\left[\min \left(n \alpha, \frac{1-\alpha}{M}\right)\right]|u|, \forall u \in[-M, M] .
$$

3. Choosing $\alpha=\frac{1}{2}$, we get

(a)

$$
\left|e^{n u}-1\right| \geq \frac{1}{2 M}|u|, \forall n \geq \frac{1}{M}, \forall u \in[-M, M] .
$$

(b)

$$
\frac{1}{2 M} \leq n \eta^{2}(n u), \quad \forall n \geq \frac{1}{M}, \forall u \in[-M, M] .
$$

(c) Let $V$ be Hermitian space. Then

$$
\frac{1}{2 M}\|x\|^{2} \leq n\|\eta(n h)(x)\|^{2}
$$

for all $n \geq \frac{1}{M}$ and for every Hermitian endomorphism $h \in \operatorname{Herm}(V)$ with the property $\operatorname{Spec}(h) \subset[-M, M]$. 
Proof: The first statements are straightforward. The last one follows from the previous ones and Remark 5.15 in the following way: we take

$$
f:=\sqrt{\frac{1}{2 M}} \mathrm{id}_{V}, g(t):=\sqrt{n} \eta(n t),
$$

and we notice that $|f|_{[-M, M]} \leq|g|_{[-M, M]}$ by 3 b.

We divide (34) by $\|s\|_{L^{2}}$, taking into account Remark $4.153 \mathrm{c}$ and (56). We get, for $\|s\|_{L^{2}} \geq \max \left(\frac{1}{M}, 1\right)$,

$$
\begin{gathered}
\frac{1}{2 M}\left|\partial_{0} \sigma\right|^{2} \leq \\
\leq s \|\left|\eta(\|s\|[\sigma, \cdot])\left(\partial_{0} \sigma\right)\right|^{2}=\frac{1}{\|s\|} \mid \eta\left(\left.[s, \cdot]\left(\partial_{0} s\right)\right|^{2} \leq\right. \\
\leq \frac{1}{\|s\|}\left[-\frac{1}{2} P\left(|s|^{2}\right)-\left(k^{0}, s\right)\right] .
\end{gathered}
$$

Integrating on $X$ the obtained inequality, we get

$$
\left\|\partial_{0} \sigma\right\|_{L^{2}}^{2} \leq 2 M\left\|k^{0}\right\|_{L^{2}} .
$$

Concluding, we can state

Proposition 4.16 Suppose that $\left(s_{\varepsilon}\right)_{\varepsilon \in(0,1]}$ is not $L^{2}$-bounded. Then the set

$$
\left\{\sigma_{\varepsilon} \mid\left\|s_{\varepsilon}\right\|_{L^{2}} \geq \max \left(\frac{1}{M}, 1\right)\right\}
$$

is uniformly bounded in $L^{\infty} \cap L_{1}^{2}$. In particular, there exists a sequence $\left(\varepsilon_{n}\right)_{n}$, $\varepsilon_{n} \rightarrow 0$ such that

1. $\lim _{n \rightarrow \infty}\left\|s_{\varepsilon_{n}}\right\|_{L^{2}}=\infty$.

2. The sequence $\left(\sigma_{\varepsilon_{n}}\right)_{n}$ converges

- weakly in $L_{1}^{2}$,

- strongly in $L^{2}$,

- almost everywhere on $X$

to an $L_{1}^{2} \cap L^{\infty}$ - section $\sigma$ in the bundle $\hat{P}^{0} \times_{\mathrm{ad}} i \mathfrak{k}_{\beta}^{\perp}$. 


\subsubsection{The properties of the limit $\sigma$}

The aim of this paragraph is the following

\section{Theorem 4.17}

1. $\|\sigma\|_{L^{2}}=1$.

2. The map $X \ni x \mapsto[\sigma(x)] \in i \mathfrak{k} / \operatorname{ad}_{\hat{K}}$ which assigns to every $x \in X$ the conjugacy class of $\sigma(x)$, is constant almost everywhere.

3. $\operatorname{pr}_{V_{\sigma(x)}^{+}}(\bar{\partial} \sigma)=0$ for almost every $x \in X$, where $V_{\sigma(x)}^{+}$is the direct sum of the eigenspaces corresponding to strictly positive eigenvalues of the endomorphism $[\sigma(x), \cdot]$ on $\mathfrak{g}_{x}:=\hat{P}_{x} \times_{\text {ad }} \mathfrak{g}$.

In order to prove this, we will need several preparations:

Let $s \in A^{0}\left(\hat{P} \times_{\text {ad }} i \mathfrak{k}\right)$ be a smooth section. Denote by $V_{s}^{0}$ the linear fibration $\bigcup_{x \in X} V_{s, x}^{0}$, where $V_{s, x}^{0}=\operatorname{ker}\left[s_{x}, \cdot\right] \subset \hat{P}_{x} \times_{\text {ad }} \mathfrak{g}$.

Let $X_{k}^{s}$ be the closed subset of $X$ where $\operatorname{ker}\left[s_{x}, \cdot\right]$ has rank $\leq k$. Then $\operatorname{ker}([s, \cdot])$ defines a continuous subbundle of $\hat{P} \times$ ad $\left.\mathfrak{g}\right|_{X_{k}^{s} \backslash X_{k-1}^{s}}$. Therefore, the projection $p_{V_{s}^{0}}$ is continuous on every $X_{k}^{s} \backslash X_{k-1}^{s}$, hence it is a bounded measurable section.

With these remarks, we can state

Proposition 4.18 Let $\hat{A}^{0} \in \mathcal{A}(\hat{P})$ and let $\left(\sigma_{n}\right)_{n}, \sigma_{n} \in A^{0}\left(\hat{P} \times_{\text {ad }} i \mathfrak{k}\right)$ be a sequence of smooth sections with the following properties:

1. It is weakly convergent in $L_{1}^{2}$.

2. It is bounded in $L^{\infty}$.

3. It converges strongly in $L^{2}$.

4. It converges almost everywhere on $X$.

5. $\left(\left\|\operatorname{pr}_{V_{\sigma_{n}}^{0}}\left[\partial_{0} \sigma_{n}\right]\right\|_{L^{2}}\right)_{n}$ converges to 0 .

Then the weak limit $\sigma$ of this sequence (which belongs to $L_{1}^{2} \cap L^{\infty}$ ) defines an almost everywhere constant map $x \mapsto[\sigma(x)] \in \mathfrak{k} / \hat{K}$.

Proof: Let Let $\iota_{1}, \ldots, \iota_{k} \in \mathbb{C}[\hat{\mathfrak{g}}]$ be homogeneous generators of the invariant algebra $\mathbb{C}[\hat{\mathfrak{g}}]^{\hat{G}}$. We will prove that $\iota_{i}(\sigma)$ are constant almost everywhere, and the claim will follow from Corollary 5.8 in the Appendix (compare with the proof of 5.9). 
Let $j_{i}: \hat{\mathfrak{g}}^{d_{i}} \rightarrow \mathbb{C}$ be the ad $\hat{G}^{\text {-invariant symmetric multilinear map which }}$ corresponds to $\iota_{i}$

Using properties 2. and 3. and 4. one gets easily, by the Lebesgue dominant convergence theorem, that $\iota_{i}\left(\sigma_{n}\right)=j_{i}\left(\sigma_{n}, \ldots, \sigma_{n}\right)$ converges to $\iota_{i}(\sigma)$ strongly in $L^{2}$.

One has

$$
\partial\left[j_{i}\left(\sigma_{n}, \ldots, \sigma_{n}\right)\right]=d_{i} j_{i}\left(\partial_{0} \sigma_{n}, \sigma_{n}, \ldots, \sigma_{n}\right) .
$$

On the other hand, by 2 . and 3 . the sequence of sections

$$
j_{i}\left(\cdot, \sigma_{n}, \ldots, \sigma_{n}\right) \in A^{0}(\hat{P} \times \text { ad } \operatorname{Hom}(i \mathfrak{k}, \mathbb{C}))
$$

is $L^{\infty}$ - bounded and converges strongly in $L^{2}$ to $j_{i}(\cdot, \sigma, \ldots, \sigma)$. Taking into account 1., 2. and 3. we deduce by Lemma 5.17 in Appendix that

$$
d_{i} j_{i}\left(\partial_{0} \sigma_{n}, \sigma_{n}, \ldots, \sigma_{n}\right) \rightarrow d_{i} j_{i}\left(\partial_{0} \sigma, \sigma, \ldots, \sigma\right) \text { weakly in } L^{2} \text {. }
$$

On the other hand $d_{i} j_{i}\left(\partial_{0} \sigma, \sigma, \ldots, \sigma\right)=\partial\left[j_{i}(\sigma, \ldots, \sigma)\right]$ as distributions because Leibniz's rule extends to $L_{1}^{2} \cap L^{\infty}$-sections. ${ }^{3}$

Therefore,

$$
d_{i} j_{i}\left(\partial_{0} \sigma_{n}, \sigma_{n}, \ldots, \sigma_{n}\right) \rightarrow \partial\left[j_{i}(\sigma, \ldots, \sigma)\right] \text { weakly in } L^{2} .
$$

On the other hand, taking into account that $j_{i}$ is ad $\hat{G}_{\hat{G}}$-invariant (hence in particular $\operatorname{ad}_{G}$-invariant), it follows easily that, for any $v \in \mathfrak{g}$, the linear functional $j_{i}(\cdot, v, \ldots, v)$ vanishes on $[v, \mathfrak{g}]$. The point is that, when $v \in i \mathfrak{k}$, one has an orthogonal decomposition

$$
\mathfrak{g}=[v, \mathfrak{g}] \oplus z_{\mathfrak{g}}(v) .
$$

This follows from the fact that, in this case, $z_{\mathfrak{g}}(v)$ is just the complexification of $z_{\mathfrak{k}}(i v)$.

Therefore one gets pointwise

$$
d_{i} j_{i}\left(\partial_{0} \sigma_{n}, \sigma_{n}, \ldots, \sigma_{n}\right)=d_{i} j_{i}\left(\operatorname{pr}_{V_{\sigma_{n}}^{0}}\left(\partial_{0} \sigma_{n}\right), \sigma_{n}, \ldots, \sigma_{n}\right),
$$

and the right term converges to 0 in $L^{2}$ as $n \rightarrow \infty$ by 5 . and 2. By (58) we get that $\partial\left[j_{i}(\sigma, \ldots, \sigma)\right]=0$ in the week sense, hence $j_{i}(\sigma, \ldots, \sigma)$ is a constant. The claim follows now from Corollary 5.8

For an element $u \in i \mathfrak{k}$ we denote by $V_{u}^{-}$the direct sum of eigenspaces of $[u, \cdot] \in \operatorname{Herm}(\mathfrak{g})$ corresponding to strictly negative eigenvalues. Again, for a section $u \in A^{0}\left(\hat{P} \times{ }_{\text {ad }} i \mathfrak{k}\right)$, the projection $\operatorname{pr}_{V_{u}^{-}}$on the fibration defined by $u$ is in $L^{\infty}(\operatorname{Herm}(\hat{P} \times$ ad $\mathfrak{g}))$.

\footnotetext{
${ }^{3}$ This can be proved by constructing a sequence of smooth sections converging to $\sigma$ with respect to both $L_{1}^{2}$ - and $L^{p}$ - norms, for $p$ sufficiently large.
} 
Proposition 4.19 Let $\hat{A}^{0} \in \mathcal{A}(\hat{P})$ and let $\left(\sigma_{n}\right)_{n}, \sigma_{n} \in A^{0}\left(\hat{P} \times_{\text {ad }} i \mathfrak{k}\right)$ be a sequence of smooth sections with the following properties

1. It is weakly convergent in $L_{1}^{2}$.

2. It is bounded in $L^{\infty}$.

3. It converges strongly in $L^{2}$.

4. It converges almost everywhere on $X$.

5. The weak limit $\sigma$ of this sequence defines an (almost everywhere) constant conjugacy class $[\sigma] \in i \mathfrak{k} / \hat{K}$.

6. $\left(\left\|\operatorname{pr}_{V_{\sigma_{n}}^{-}}\left[\partial_{0} \sigma_{n}\right]\right\|_{\left.L^{2}\right)_{n}}\right.$ converges to 0 .

Then the weak limit $\sigma$ (which belongs to $L_{1}^{2} \cap L^{\infty}$ ) satisfies

$$
\operatorname{pr}_{V_{\sigma, x}^{-}}\left[\partial_{0} \sigma\right]=0 \text { for almost every } x \in X .
$$

Proof: Let $\sigma_{0} \in i \mathfrak{k}$ be a representative of $[\sigma]$ and $\lambda_{1}<\ldots \lambda_{k}<0$ be the negative eigenvalues of $\left[\sigma_{0}, \cdot\right]$. Then $\left[\sigma_{x}, \cdot\right]$ has the same eigenvalues with the same multiplicities for almost every $x \in X$.

Indeed, one can easily see that for $u \in i \mathfrak{k}$, the eigenvalues of the endomorphism $[u, \cdot] \in \operatorname{Herm}(\mathfrak{g})$ depend only on the class $[u] \in i \mathfrak{k} / \hat{K}$. This follows from the fact that, for $\hat{k} \in \hat{K}$, one has $\left.\operatorname{ad}_{\hat{k}}(s), \cdot\right]=\operatorname{ad}_{\hat{k}}\left[s, \operatorname{ad}_{\hat{k}^{-1}}(\cdot)\right]$.

Let $\eta \in\left(\lambda_{k}, 0\right)$ and $\chi: \mathbb{R} \rightarrow[0,1]$ a smooth increasing function which is 1 on $(-\infty, \eta]$ and 0 on $[0, \infty)$.

Put

$$
q_{n}:=\chi\left(\left[\sigma_{n}, \cdot\right]\right) .
$$

Since $\sigma_{n}$ converges strongly in $L^{2}$, it follows that $q_{n}$ converges strongly in $L^{2}$ to $q:=\chi([\sigma, \cdot])=\operatorname{pr}_{V_{\sigma}^{-}}$. This follows from the Lebesgue dominated convergence theorem, from 4 . and the fact that, for any Hermitian vector space $E$, the map

$$
\chi: \operatorname{Herm}(E) \longrightarrow \operatorname{Herm}(E), f \mapsto \chi(f)
$$

is continuous.

Therefore $q_{n}\left[\partial_{0} \sigma_{n}\right]$ converges to $q\left(\partial_{0}(\sigma)\right)=\operatorname{pr}_{V_{\sigma}^{-}}\left[\partial_{0} \sigma\right]$ as distributions. On the other hand

$$
\left\|q_{n}\left[\partial_{0} \sigma_{n}\right]\right\|_{L^{2}} \leq\left\|\operatorname{pr}_{V_{\sigma_{n}}^{-}}\left[\partial_{0} \sigma_{n}\right]\right\|_{L^{2}}
$$

which converges to 0 by 6 . 
Therefore $\operatorname{pr}_{V_{\sigma}^{-}}\left[\partial_{0} \sigma\right]=0$ as a distribution, hence as an $L^{2}$ - section as well.

We can now give the

Proof (of Theorem 4.17):

We make use of Proposition 4.16 and put $s_{n}:=s_{\varepsilon_{n}} . \sigma_{n}:=\sigma_{\varepsilon_{n}}$. Since $\left\|\sigma_{n}\right\|_{L^{2}}=1$, and $\sigma_{n} \rightarrow \sigma$ strongly in $L^{2}$, we get $\|\sigma\|_{L^{2}}=1$ as claimed.

For the proof of 2 . and 3. we claim first that

$$
\left(\left\|\operatorname{pr}_{V_{-}^{\sigma_{n}}}\left[\partial_{0} \sigma_{n}\right]\right\|_{\left.L^{2}\right)_{n}} \rightarrow 0\right.
$$

where, for $s \in A^{0}\left(\hat{P} \times{ }_{\text {ad }} i \mathfrak{k}\right)$, we denoted by $V_{-}^{s}$ the fibration whose fibre in $x \in X$ is the direct sum of all eigenspaces corresponding to all non-positive eigenvalues of $\left[s_{x}, \cdot\right]$.

This follows easily in the following way: using the inequality

$$
\frac{1-e^{-t}}{t} \geq 1 \text { for } t \leq 0
$$

we see that $\eta \geq \chi_{-}$, where $\chi_{-}:=\chi_{\mathbb{R}_{\leq 0}}$ is the characteristic function of the set $\mathbb{R}_{\leq 0}$. By Remark 5.15 and (34) one gets

$$
\begin{aligned}
& \left|\operatorname{pr}_{V_{-}^{\sigma_{n}}}\left[\partial_{0} \sigma_{n}\right]\right|_{x}^{2}=\frac{1}{\left\|s_{n}\right\|^{2}}\left|\operatorname{pr}_{V_{-}^{s_{n}}}\left[\partial_{0} s_{n}\right]\right|_{x}^{2}=\frac{1}{\left\|s_{n}\right\|^{2}} \mid\left(\left.\chi_{-}\left(\left[s_{n}, \cdot\right]\right)\left(\partial_{0} s_{n}\right)\right|_{x} ^{2} \leq\right. \\
& \leq \frac{1}{\left\|s_{n}\right\|^{2}} \mid\left(\left.\eta\left(\left[s_{n}, \cdot\right]\right)\left(\partial_{0} s_{n}\right)\right|_{x} ^{2} \leq \frac{1}{\left\|s_{n}\right\|^{2}}\left[-\frac{1}{2} P\left(\left|s_{n}\right|^{2}\right)-\varepsilon\left|s_{n}\right|^{2}-\left(k^{0}(x), s_{n}\right)\right](x) .\right.
\end{aligned}
$$

It suffices to integrate the obtained inequality over $X$, and let $\varepsilon \rightarrow 0$ taking into account that $\left\|s_{n}\right\| \rightarrow \infty$.

In particular, it follows that

$$
\left(\left\|\operatorname{pr}_{V_{\sigma_{n}}^{0}}\left[\partial_{0} \sigma_{n}\right]\right\|_{L^{2}}\right)_{n} \rightarrow 0,
$$

hence, by Propositions 4.18, the limit $\sigma$ defines an almost everywhere constant map $X \rightarrow \mathfrak{i k} / \hat{K}$. Therefore, we can apply Proposition 4.19, because (59) obviously implies $\left\|\operatorname{pr}_{V_{\sigma_{n}}^{-}}\left[\partial_{0} \sigma_{n}\right]\right\|_{L^{2}} \rightarrow 0$.

\subsubsection{The limit $\sigma$ destabilizes}

Let $\sigma$ be the limit given by Proposition 4.16. 
Proposition 4.20 Let $[\sigma] \subset i \mathfrak{k}$ be the element in $i \mathfrak{k} / \hat{K}$ defined by the (almost everywhere constant) map $x \mapsto\left[\sigma_{x}\right]$ (modulo ad ${ }_{\hat{K}}$ ) and let $\sigma_{0} \in i \mathfrak{k}_{\beta}^{\perp} \backslash\{0\}$ be any representative of $[\sigma]$. Then:

1. The $L_{1}^{2} \cap L^{\infty}$ section $\sigma$ is smooth and takes values in $[\sigma]$ on the complement $X_{\rho}$ of a Zariski subset of codimension at least 2 such that the subspace

$$
\left\{\left.q \in \hat{\mathcal{Q}}\right|_{X_{\rho}}, \sigma(q) \in \hat{\mathfrak{g}}_{\sigma_{0}}\right\}
$$

extends to a meromorphic reduction $\hat{\mathcal{Q}}^{\rho} \hookrightarrow \hat{\mathcal{Q}}$ of $\hat{\mathcal{Q}}$ to $\hat{G}_{\sigma_{0}}$ which is holomorphic over $X_{\rho}$.

2. The section $s\left(\sigma_{0}, \rho\right)$ associated with $\sigma_{0}$ and the $Z_{\hat{K}}\left(\sigma_{0}\right)$-reduction $\hat{\mathcal{Q}}^{\rho} \cap \hat{P}$ of $\left.\hat{P}\right|_{X_{\rho}}$ coincides with $\sigma$. More precisely, $\sigma(q)=\sigma_{0}$ for all $q \in \hat{\mathcal{Q}}^{\rho} \cap \hat{P}$.

Proof: This follows from Proposition 4.17 and Proposition 5.27. There is one simple detail to be explained. The weak holomorphy condition in Proposition 5.27 reads in our case

$$
\operatorname{pr}_{V_{\hat{\sigma}(x)}^{+}}\left(\bar{\partial}_{x} \hat{\sigma}\right)=0 \text { for almost every } x \in X
$$

where $\hat{\sigma}$ is the section in $\hat{P} \times{ }_{\text {ad }} i \hat{\mathfrak{g}}=i \operatorname{ad}(\hat{P})$ defined by $\sigma$, and $V_{\hat{\sigma}(x)}^{+}$is the direct sum of strictly positive eigenspaces of $[\hat{\sigma}(x), \cdot] \in \operatorname{Herm}\left(\hat{\mathfrak{g}}_{x}\right)$.

But, since

$$
\bar{\partial} \hat{\sigma} \in L^{2}\left(\Lambda^{01}(\hat{P} \times \text { ad } \mathfrak{g})\right), V_{\hat{\sigma}(x)}^{+}=V_{\sigma(x)}^{+} \subset \mathfrak{g}_{x},
$$

this condition is equivalent to

$$
\operatorname{pr}_{V_{\sigma(x)}^{+}}\left(\bar{\partial}_{x} \sigma\right)=0 \text { for almost every } x \in X .
$$

The following result will end the proof of Theorem 4.2.

Theorem 4.21 The pair $\left(\rho, \frac{\sigma_{0}}{2}\right)$ destabilizes the pair $(\hat{J}, \varphi)$.

The first step is to give an explicit formula for the total maximal weight

$$
\frac{2 \pi}{(n-1) !} \operatorname{deg}_{g}\left(\rho, h\left(\frac{\sigma_{0}}{2}\right)\right)+\int_{X_{0}} \lambda^{\frac{\sigma_{0}}{2}}(\varphi, \rho) \operatorname{vol}_{g} .
$$


For the first term, we obtain by (14)

$$
\begin{gathered}
\frac{2 \pi}{(n-1) !} \operatorname{deg}_{g}\left(\rho, h\left(\frac{\sigma_{0}}{2}\right)\right)=\frac{1}{2(n-1) !} \int_{X} h\left(\left[i F_{\hat{A}^{0}}-\frac{i}{2}[a \wedge a]\right], \sigma\right) \wedge \omega_{g}^{n-1}= \\
\frac{1}{2} \int_{X} h\left(\Lambda_{g}\left[i F_{\hat{A}^{0}}-\frac{i}{2}[a \wedge a]\right], \sigma\right) \wedge v^{\prime} l_{g}
\end{gathered}
$$

where $a$ is the second fundamental form of the $\hat{K}_{\sigma_{0}}=\hat{G}\left(\sigma_{0}\right) \cap \hat{K}$-bundle $\hat{\mathcal{Q}}^{\rho} \cap \hat{P}$ in $\left.\hat{P}\right|_{X_{\rho}}$.

By (19) we have

$$
\operatorname{pr}_{\hat{\mathfrak{k}}_{\sigma_{0}}}[a \wedge a]=-2 \sum_{\lambda \in \operatorname{Spec}^{+}\left[\sigma_{0}, \cdot\right]}\left[a_{\lambda} \wedge \bar{a}_{\lambda}\right],
$$

so using the known formula (21), we get

$$
\begin{gathered}
h\left(\frac{i}{2} \Lambda_{g} \operatorname{pr}_{\hat{\mathfrak{k}}_{\sigma_{0}}}[a \wedge a], \sigma\right)=-\sum_{\lambda \in \operatorname{Spec}^{+}\left[\sigma_{0}, \cdot\right]} i \Lambda_{g} h\left(\left[a_{\lambda} \wedge \bar{a}_{\lambda}\right], \sigma\right)= \\
=-\sum_{\lambda \in \operatorname{Spec}^{+}\left[\sigma_{0}, \cdot\right]} i \lambda \Lambda_{g} h\left(a_{\lambda} \wedge \bar{a}_{\lambda}\right\rangle=-\sum_{\lambda \in \operatorname{Spec}^{+}\left[\sigma_{0}, \cdot\right]} \lambda\left|a_{\lambda}\right|^{2} .
\end{gathered}
$$

The $(1,0)$-form $\partial_{0} \sigma$ has non-trivial components only on the eigenspaces of $\left[\sigma_{0}, \cdot\right]$ associated with strictly positive eigenvalues. Indeed, since the conjugacy class of $\sigma$ is constant, $\partial_{0} \sigma$ has no $\operatorname{ker}\left[\sigma_{0}, \cdot\right]$ - component by Proposition 5.9. On the other hand, by the holomorphy of $\left.\hat{\mathcal{Q}}^{\rho}\right|_{X_{\rho}}$ it follows that $a_{\lambda}^{10}=0$ for $\lambda \in \operatorname{Spec}^{-}\left[\sigma_{0}, \cdot\right]$ (see formula (18), Proposition 5.26).

Therefore

$$
\partial_{0} \sigma=\sum_{\lambda \in \operatorname{Spec}^{+}\left[\sigma_{0}, \cdot\right]}\left[a_{\lambda}, \sigma\right]=-\sum_{\lambda \in \operatorname{Spec}^{+}\left[\sigma_{0}, \cdot\right]} \lambda a_{\lambda} .
$$

Let $\phi$ be the real function defined by

$$
\phi(\tau)=\left\{\begin{array}{ccc}
\frac{1}{\sqrt{\tau}} & \text { if } & \tau>0 \\
0 & \text { if } & \tau \leq 0 .
\end{array}\right.
$$

We get

$$
\phi([\sigma, \cdot])\left(\partial_{0} \sigma\right)=-\sum_{\lambda \in \operatorname{Spec}^{+}\left[\sigma_{0}, \cdot\right]} \sqrt{\lambda} a_{\lambda},
$$

so, comparing with (60), one can write

$$
h\left(\frac{i}{2} \Lambda_{g} \operatorname{pr}_{\hat{\mathfrak{k}}_{\sigma_{0}}}[a \wedge a], \sigma\right)=-\left|\phi\left(\left[\sigma_{0}, \cdot\right]\right)\left(\partial_{0} \sigma\right)\right|^{2} .
$$


Since the (1,0)-form $\partial_{0} \sigma$ has non-trivial components only on the eigenspaces of $[\sigma, \cdot]$ associated with strictly positive eigenvalues, the right hand term is indeed smooth on $X_{\rho}$.

Therefore, we obtain for the total maximal weight of the pair $(\hat{J}, \varphi)$ the formula

$$
\begin{aligned}
& \lambda\left((\hat{J}, \varphi),\left(\rho, \frac{\sigma_{0}}{2}\right)\right):=\frac{2 \pi}{(n-1) !} \operatorname{deg}_{g}\left(\rho, h\left(\frac{\sigma_{0}}{2}\right)\right)+\int_{X_{\rho}} \lambda^{\frac{\sigma_{0}}{2}}(\varphi, \rho) \operatorname{vol}_{g}= \\
& =\frac{1}{2}\left[\left\langle i \Lambda_{g} F_{\hat{A}^{0}}, \sigma\right\rangle_{L^{2}}+\left\|\phi([\sigma, \cdot])\left(\partial_{0} \sigma\right)\right\|_{L^{2}}^{2}\right]+\int_{X_{\rho}} \lim _{t \rightarrow \infty} \mu^{\frac{-i \sigma}{2}}\left(e^{t \frac{\sigma}{2}} \varphi\right) .
\end{aligned}
$$

Lemma 4.22 Let $\left(\rho, \sigma_{0}\right)$ be any pair consisting of an element $\sigma_{0} \in i \mathfrak{k}$ and a meromorphic reduction of $\hat{\mathcal{Q}}$ to $\hat{G}\left(\sigma_{0}\right)$. Fix a $\hat{K}$-reduction $\hat{P}$ of $\hat{\mathcal{Q}}$, let $\sigma$ be the section in $A^{0}\left(\hat{P} \times_{\text {ad }} i \mathfrak{k}\right)$ which corresponds to $\sigma_{0}$ and this reduction. Let $\hat{A}^{0} \in \mathcal{A}(\hat{P})$ be the Chern connection of $\hat{P}$ and $d_{\hat{A}^{0}}=\bar{\partial}+\partial_{0}$ the associated covariant derivative. Then

$$
\begin{gathered}
\lambda\left((\hat{J}, \varphi),\left(\rho, \frac{\sigma_{0}}{2}\right)\right)= \\
\frac{1}{2}\left\langle i \Lambda_{g} F_{\hat{A}^{0}}, \sigma\right\rangle_{L^{2}}+\lim _{t \rightarrow \infty} \int_{X_{\rho}}\left[\frac{1}{2} t\left|\eta([t \sigma, \cdot])\left(\partial_{0} \sigma\right)\right|^{2}+\mu^{-i \frac{\sigma}{2}}\left(e^{t \frac{\sigma}{2}} \varphi\right)\right] \operatorname{vol}_{g} .
\end{gathered}
$$

Proof: It suffices to prove that

$$
\left\|\phi([\sigma, \cdot])\left(\partial_{0}(\sigma)\right)\right\|_{L^{2}}^{2}=\lim _{t \rightarrow \infty} \int_{X_{\rho}} t\left|\eta([t \sigma, \cdot])\left(\partial_{0} \sigma\right)\right|^{2} \operatorname{vol}_{g} .
$$

For $t>0$, consider the real analytic function $\eta_{t}$ defined by

$$
\eta_{t}(\tau)=\sqrt{t} \eta(t \tau)=\left\{\begin{array}{ccc}
\sqrt{\frac{1-e^{-t \tau}}{\tau}} & \text { if } & \tau \neq 0 \\
\sqrt{t} & \text { if } & \tau=0 .
\end{array}\right.
$$

Recall that the conjugacy class of $\sigma$ is constant, so the eigenvalues of $[\sigma, \cdot]$ are also constant (see the proof of Proposition 4.19). Let $\lambda_{0}$ be the first positive eigenvalue of $[\sigma, \cdot]$. Note that, by Proposition 5.9 and Theorem 5.26, the form $\left.\partial_{0}(\sigma)\right|_{X_{\rho}}$ has non-trivial components only along the strictly positive eigenspaces of $[\sigma, \cdot]$. Therefore, pointwise on $X_{\rho}$ one has

$$
\left.|t| \eta([t \sigma, \cdot])\left(\partial_{0} \sigma\right)\right|^{2}-\left|\phi([\sigma, \cdot])\left(\partial_{0} \sigma\right)\right|^{2}\left|\leq \sup _{\left[\lambda_{0}, \infty\right]}\right| \eta_{t}^{2}-\left.\phi^{2}|| \partial_{0} \sigma\right|^{2} .
$$


The point is now that $\eta_{t}^{2}$ converges uniformly to $\phi^{2}$ on the interval $\left[\lambda_{0}, \infty\right)$ as $t \rightarrow \infty$. Integrating both sides on $X_{\rho}$ we get the result.

Let $\sigma_{n}=\frac{1}{\left\|s_{n}\right\|_{L^{2}}} s_{n}$ be the sequence with limit $\sigma$ given by Proposition 4.16 .

Lemma 4.23 Fix $t_{0}>0$. Then

$$
\begin{gathered}
\left\langle i \Lambda_{g} F_{\hat{A}^{0}}, \sigma\right\rangle_{L^{2}}+\int_{X_{\rho}}\left[\frac{1}{2} t_{0}\left|\eta\left(\left[t_{0} \sigma, \cdot\right]\right)\left(\partial_{0} \sigma\right)\right|^{2}+\mu^{-i \frac{\sigma}{2}}\left(e^{t_{0} \frac{\sigma}{2}} \varphi\right)\right] \operatorname{vol}_{g} \leq \\
\liminf _{n \rightarrow \infty}\left\{\left\langle i \Lambda_{g} F_{\hat{A}^{0}}, \sigma_{n}\right\rangle_{L^{2}}+\int_{X_{\rho}}\left[\frac{1}{2} t_{0}\left|\eta\left(\left[t_{0} \sigma_{n}, \cdot\right]\right)\left(\partial_{0} \sigma_{n}\right)\right|^{2}+\mu^{-i \frac{\sigma_{n}}{2}}\left(e^{t_{0} \frac{\sigma_{n}}{2}} \varphi\right)\right] \text { vol }_{g}\right\} .
\end{gathered}
$$

Proof: First of all notice that

$$
\left\langle i \Lambda_{g} F_{\hat{A}^{0}}, \sigma_{n}\right\rangle_{L^{2}} \rightarrow\left\langle i \Lambda_{g} F_{\hat{A}^{0}}, \sigma\right\rangle_{L^{2}}
$$

because $\left(\sigma_{n}\right)_{n}$ converges strongly in $L^{2}$ to $\sigma$.

Second, one has

$$
\begin{gathered}
\mu^{-i \frac{\sigma}{2}}\left(e^{t_{0} \frac{\sigma}{2}} \varphi\right)=\left\langle\mu(\varphi),-i \frac{\sigma}{2}\right\rangle+\left[\mu^{-i \frac{\sigma}{2}}\left(e^{t_{0} \frac{\sigma}{2}} \varphi\right)-\mu^{-i \frac{\sigma}{2}}(\varphi)\right], \\
\mu^{-i \frac{\sigma_{n}}{2}}\left(e^{t_{0} \frac{\sigma_{n}}{2}} \varphi\right)=\left\langle\mu(\varphi),-i \frac{\sigma_{n}}{2}\right\rangle+\left[\mu^{-i \frac{\sigma_{n}}{2}}\left(e^{t_{0} \frac{\sigma_{n}}{2}} \varphi\right)-\mu^{-i \frac{\sigma_{n}}{2}}(\varphi)\right] .
\end{gathered}
$$

Since $\sigma_{n} \rightarrow \sigma$ strongly in $L^{2}$, it follows that

$$
\int_{X_{\rho}}\left\langle\mu(\varphi),-i \frac{\sigma_{n}}{2}\right\rangle \operatorname{vol}_{g} \rightarrow \int_{X_{\rho}}\left\langle\mu(\varphi),-i \frac{\sigma}{2}\right\rangle v{ } l_{g} .
$$

The two terms $\left[\mu^{-i \frac{\sigma_{n}}{2}}\left(e^{t_{0} \frac{\sigma_{n}}{2}} \varphi\right)-\mu^{-i \frac{\sigma_{n}}{2}}(\varphi)\right],\left[\mu^{-i \frac{\sigma}{2}}\left(e^{t_{0} \frac{\sigma}{2}} \varphi\right)-\mu^{-i \frac{\sigma}{2}}(\varphi)\right]$ are pointwise non-negative, because the value in every point $x \in X$ can be identified with the energy of a curve in the fibre $F_{x}$. On the other hand, the first term converges almost everywhere to the second as $n \rightarrow \infty$. By Fatou's lemma, it follows that

$$
\begin{gathered}
\int_{X_{\rho}}\left[\mu^{-i \frac{\sigma}{2}}\left(e^{t_{0} \frac{\sigma}{2}} \varphi\right)-\mu^{-i \frac{\sigma}{2}}(\varphi)\right] v l_{g} \leq \\
\leq \liminf _{n \rightarrow \infty} \int_{X_{\rho}}\left[\mu^{-i \frac{\sigma_{n}}{2}}\left(e^{t_{0} \frac{\sigma_{n}}{2}} \varphi\right)-\mu^{-i \frac{\sigma_{n}}{2}}(\varphi)\right] .
\end{gathered}
$$


Finally we state that

$$
\int_{X_{\rho}}\left[\left|\eta\left(\left[t_{0} \sigma, \cdot\right]\right)\left(\partial_{0} \sigma\right)\right|^{2}\right] v o l_{g} \leq \liminf _{t \rightarrow \infty} \int_{X_{\rho}}\left[\left|\eta\left(\left[t_{0} \sigma_{n}, \cdot\right]\right)\left(\partial_{0} \sigma_{n}\right)\right|^{2}\right] \operatorname{vol}_{g} .
$$

Indeed, since $\left(\eta\left(\left[t_{0} \sigma_{n}, \cdot\right]\right)\right)_{n}$ converges strongly in $L^{2}$ to $\eta\left(\left[t_{0} \sigma_{n}, \cdot\right]\right)$ and is $L^{\infty}$-bounded, and since $\partial_{0}(\sigma)$ converges weakly in $L^{2}$ to $\partial_{0} \sigma$, it follows that $\eta\left(\left[t_{0} \sigma_{n}, \cdot\right]\right)\left(\partial_{0} \sigma_{n}\right)$ converges weakly to $\eta\left(\left[t_{0} \sigma, \cdot\right]\right)\left(\partial_{0} \sigma\right)$ in $L^{2}$ (see Lemma 5.17 in the Appendix).

Now the inequality (66) follows from the well known semicontinuity property of the norm with respect to the weak convergence in a Hilbert space. The claim of our lemma follows from (63), (64), (65) and (66).

Lemma 4.24 Let $\mathfrak{s} \in A^{0}\left(\hat{P} \times_{\text {ad }} i \mathfrak{k}\right)$. Then the maps

$$
\begin{gathered}
t_{0} \mapsto t_{0}\left|\eta\left(\left[t_{0} \mathfrak{s}, \cdot\right]\right)\left(\partial_{0} \mathfrak{s}\right)\right|^{2}, \\
t_{0} \mapsto \mu_{0}^{-i \frac{\mathfrak{s}}{2}}\left(e^{t_{0} \frac{\mathfrak{s}}{2}} \varphi\right)
\end{gathered}
$$

are pointwise increasing.

Proof: The second map is obviously pointwise increasing (see Remark 1.6). For the first, it suffices to notice that the function $\eta_{t_{0}}$ defined by (62) takes positive values and is increasing with respect to $t_{0}$. The result follows from Remark 5.15. We can now give the

Proof (of Theorem 4.21):

If not, one would have

$$
\lambda\left((\hat{J}, \varphi),\left(\rho, \frac{\sigma_{0}}{2}\right)\right)>0 .
$$

By Lemma 4.22, it would follow that there exists $t_{0}>0$ such that

$$
U:=\frac{1}{2}\left\langle i \Lambda_{g} F_{\hat{A}^{0}}, \sigma\right\rangle_{L^{2}}+\int_{X_{\rho}}\left[\frac{1}{2} t_{0}\left|\eta\left(\left[t_{0} \sigma, \cdot\right]\right)\left(\partial_{0} \sigma\right)\right|^{2}+\mu^{-i \frac{\sigma}{2}}\left(e^{t_{0} \frac{\sigma}{2}} \varphi\right)\right] \operatorname{vol}_{g}>0 .
$$

By Lemma 4.23 we get that for all sufficiently large $n \in \mathbb{N}$, we have

$$
\frac{1}{2}\left\langle i \Lambda_{g} F_{\hat{A}^{0}}, \sigma_{n}\right\rangle_{L^{2}}+\int_{X}\left[\frac{1}{2} t_{0}\left|\eta\left(\left[t_{0} \sigma_{n}, \cdot\right]\right)\left(\partial_{0} \sigma_{n}\right)\right|^{2}+\mu^{-i \frac{\sigma_{n}}{2}}\left(e^{t_{0} \frac{\sigma_{n}}{2}} \varphi\right)\right] \operatorname{vol}_{g}>\frac{U}{2} .
$$


Choose $n$ sufficiently large such that $\left\|s_{n}\right\|_{L^{2}}>t_{0}$. By the monotony Lemma 4.24 , we get that

$$
\begin{gathered}
\frac{1}{2}\left\langle i \Lambda_{g} F_{\hat{A}^{0}}, \sigma_{n}\right\rangle_{L^{2}} \\
+\int_{X}\left[\frac{1}{2}\left\|s_{n}\right\|\left|\eta\left(\left[\left\|s_{n}\right\| \sigma_{n}, \cdot\right]\right)\left(\partial_{0} \sigma_{n}\right)\right|^{2}+\mu^{-i \frac{\sigma_{n}}{2}}\left(e^{\left\|s_{n}\right\| \frac{\sigma_{n}}{2}} \varphi\right)\right] \operatorname{vol}_{g}>\frac{U}{2}
\end{gathered}
$$

or, equivalently,

$$
\frac{1}{\left\|s_{n}\right\|}\left\{\frac{1}{2}\left\langle i \Lambda_{g} F_{\hat{A}^{0}}, s_{n}\right\rangle_{L^{2}}+\int_{X}\left[\frac{1}{2}\left|\eta\left(\left[s_{n}, \cdot\right]\right)\left(\partial_{0} s_{n}\right)\right|^{2}+\mu^{-i \frac{s_{n}}{2}}\left(e^{\frac{s_{n}}{2}} \varphi\right)\right] \operatorname{vol}_{g}\right\}>\frac{U}{2} .
$$

But, integrating (32) on $X$ and taking into account the identity given by Proposition 5.16, we get

$$
\begin{gathered}
\frac{1}{\left\|s_{n}\right\|}\left\{\frac{1}{2}\left\langle i \Lambda_{g} F_{\hat{A}^{0}}, s_{n}\right\rangle_{L^{2}}+\int_{X}\left[\frac{1}{2}\left|\eta\left(\left[s_{n}, \cdot\right]\right)\left(\partial_{0} s_{n}\right)\right|^{2}+\mu^{-i \frac{s_{n}}{2}}\left(e^{\frac{s_{n}}{2}} \varphi\right)\right] \operatorname{vol}_{g}\right\}= \\
=-\frac{\varepsilon\left\|s_{n}\right\|}{2} .
\end{gathered}
$$

We now can finally give the proof of our main result.

Proof (of Theorem 4.2): Theorem 4.14 yields a Hermitian-Einstein reduction $\hat{P}_{s}$, unless the smooth family $\left(s_{\varepsilon}\right)_{\varepsilon \in(0,1]}$ given by this theorem is not $L^{2}$ bounded. In this case Proposition 4.16 applies and one obtains a pair $\left(\rho, \frac{\sigma_{0}}{2}\right)$ with $\lambda\left((\hat{J}, \varphi),\left(\rho, \frac{\sigma_{0}}{2}\right)\right) \leq 0$ by Theorem 4.21. But, using Remark 2.20 and the fact that $\sigma_{0} \in i \mathfrak{k}_{\beta}^{\perp} \backslash\{0\}$, this contradicts polystability.

\section{Appendix}

\subsection{Chern connections}

Let $G$ be a complex Lie group and $Q$ a principal $G$-bundle over a complex manifold $M$. By definition, an (almost) holomorphic structure in $Q$ is an (almost) complex structure in the total space of $Q$ which makes the projection on $M$ (almost) holomorphic and the action $G \times Q \rightarrow Q$ holomorphic. In other words, 
an (almost) holomorphic structure in the principal bundle $Q$ is a $G$-invariant (almost) complex structure on its total space which agrees with the complex structure induced by the complex structure in the Lie algebra $\mathfrak{g}$ on the vertical subbundle $V_{Q} \subset T_{Q}$, and with the complex structure in $M$ on the quotient bundle $T_{Q} / V_{Q}$.

A holomorphic structure on $Q$ can be alternatively defined as the data of a maximal system of trivializations with holomorphic transition functions.

If $K$ is a maximal compact subgroup of a reductive group $G$ and $P \subset Q$ is a $K$-reduction of $Q$, then the Chern correspondence identifies the space $\overline{\mathcal{A}}(Q)$ of almost holomorphic structures on the bundle $Q$ with the space $\mathcal{A}(P)$ ) of connections on $P$.

The correspondence is defined as follows: if $J$ is an almost complex structure on the $G$-bundle $Q$, then the horizontal spaces of the associated connection $A \in \mathcal{A}(P)$ are given by:

$$
T_{p}^{\text {horiz }}(P)=T_{p}(P) \cap J\left(T_{p}(P)\right), p \in P .
$$

The tangent space $T_{p}(Q)$ decomposes as

$$
T_{p}(Q)=T_{p}(P) \oplus T_{p}(P)^{\perp}=T_{p}^{\text {horiz }}(P) \oplus T_{p}^{\mathrm{vert}}(P) \oplus T_{p}(P)^{\perp},
$$

where $T_{p}(P)^{\perp}=\left\{a_{p}^{\#} \mid a \in \mathfrak{i k}\right\}$.

The Chern correspondence identifies the space $\mathcal{H}(Q)$ of holomorphic structures on $Q$ with the space

$$
\mathcal{A}^{1,1}(P):=\left\{A \in \mathcal{A}(P) \mid F_{A} \in \mathcal{A}^{1,1}(\operatorname{ad}(P))\right\}
$$

of integrable connections on $P$.

We will denote by $J^{A}$ the (almost) holomorphic structure corresponding to the connection $A$ and by $A_{J}$ (or, more precisely $A_{P, J}$ ) the connection corresponding to $J$.

The aim of this section is to study the dependence of this connection and its curvature on $P$, when $J$ is fixed.

Proposition 5.1 The vertical component of a vector $w \in T_{p}(P)$ with respect to the Chern connection $A_{P, J}$ is given by the formula

$$
\operatorname{vert}(w)=-J\left[\operatorname{pr}_{T_{p}(P)^{\perp}}(J(w))\right] .
$$

Proof: One has

$$
J\left(T_{p}(P)^{\perp}\right)=T_{p}^{\mathrm{vert}}(P), J\left(T_{p}^{\text {horiz }}(P)\right)=T_{p}^{\text {horiz }}(P),
$$


hence

$$
w=\operatorname{horiz}(w)+\operatorname{vert}(w) ; J(w)=J \operatorname{horiz}(w)+J \operatorname{vert}(w),
$$

so that

$$
J \operatorname{vert}(w)=\operatorname{pr}_{T_{p}(P)^{\perp}}(J(w)) ; \operatorname{vert}(w)=-J\left[\operatorname{pr}_{T_{p}(P)^{\perp}}(J(w))\right] .
$$

Let $P^{0}$ be a fixed $K$-reduction of $Q$, and let $A^{0}$ be the Chern connection of the pair $\left(P^{0}, J\right)$. One can write

$$
P=e^{-\frac{s}{2}}\left(P^{0}\right)
$$

where $s \in A^{0}\left(P^{0} \times_{\text {ad }} i \mathfrak{k}\right)$.

Proposition 5.2 Denote by $A^{s}$ the Chern connection of the pair $(P, J)$. Regarding both connections $A^{s}$ and the initial connection $A^{0}:=A_{P^{0}, J}$ as connections in $Q$, the relation between these two connections is

$$
A^{s}=A^{0}+h^{-1} \partial_{0} h
$$

where $h:=e^{s}$, and the term $h^{-1} \partial_{0} h$ on the right is defined in the following way: regard $h$ as a $G$-equivariant map $\chi: Q \rightarrow G$, and define the ad-tensorial $(1,0)$ form $\chi^{-1} \partial_{0} \chi$ on $Q$ by setting

$$
\chi^{-1} \partial_{0} \chi(v)=\chi^{-1} \partial \chi\left(\operatorname{horiz}_{A^{0}}(v)\right)=\left[L_{\chi(q)^{-1}}\right]_{*} \partial \chi\left(\operatorname{horiz}_{A^{0}}(v)\right)
$$

for all $q \in Q, v \in T_{q}(Q)$. This tensorial form can be identified with a form in $h^{-1} \partial_{0} h \in A^{1,0}(X, \operatorname{ad}(Q))$.

Proof: Put $k:=e^{-\frac{s}{2}}$. Choose $p \in P^{0}$, and let $q:=k(p)=p \kappa(p) \in P^{s}$. We want to compute the difference $\alpha:=\theta_{A^{s}}-\theta_{A^{0}}$ between the two connection forms in the point $q$.

Fix an $A^{0}$-horizontal vector $w \in T_{p}\left(P^{0}\right)$. The vector

$$
\left[R_{\kappa(p)}\right]_{*}(w) \in T_{q}\left(R_{\kappa(p)}\left(P^{0}\right)\right) \subset T_{q}(Q)
$$

will be horizontal with respect to the same connection regarded as a connection on $Q$.

On the other hand,

$$
k_{*}(w)=\left[R_{\kappa(p)}\right]_{*}(w)+q \kappa(p)^{-1} d \kappa(w) \in T_{q}\left(P^{s}\right) .
$$


Since $J(w)$ belongs to $T_{p}\left(P^{0}\right)$ too, one has

$$
k_{*}(J w)=\left[R_{\kappa(p)}\right]_{*}(J w)+q \kappa(p)^{-1} d \kappa(J w) \in T_{q}\left(P^{s}\right),
$$

hence

$$
\left[R_{\kappa(p)}\right]_{*}(J w) \equiv-q \kappa(p)^{-1} d \kappa(J w) \bmod T_{q}\left(P^{s}\right)
$$

and

$$
\left(\theta_{A^{s}}-\theta_{A^{0}}\right)\left(k_{*}(w)\right)=\theta_{A^{s}}\left(\left[R_{\kappa(p)}\right]_{*}(w)+q \kappa(p)^{-1} d \kappa(w)\right)-\kappa(p)^{-1} d \kappa(w) .
$$

But

$$
\begin{gathered}
\operatorname{vert}_{A^{s}}\left(\left[R_{\kappa(p)}\right]_{*}(w)+q \kappa(p)^{-1} d \kappa(w)\right)= \\
=-J \operatorname{pr}_{T_{q}\left(P^{s}\right)^{\perp}}\left(\left(\left[R_{\kappa(p)}\right]_{*}(J w)+J q \kappa(p)^{-1} d \kappa(w)\right)=\right. \\
=-J \operatorname{pr}_{T_{q}\left(P^{s}\right)^{\perp}}\left(-q \kappa(p)^{-1} d \kappa(J w)+J q \kappa(p)^{-1} d \kappa(w)\right)= \\
=q\left\{-J_{\mathfrak{g}} \operatorname{pr}_{\mathfrak{k} \perp}\left(-\kappa(p)^{-1} d \kappa(J w)+J_{\mathfrak{g}}\left(\kappa(p)^{-1} d \kappa(w)\right)\right\}\right.
\end{gathered}
$$

Therefore, since $\theta_{A^{s}}-\theta_{A^{0}}$ is a tensorial form of type ad and $\left[R_{\kappa(p)}\right]_{*}(w)-k_{*}(w)$ is a vertical vector, one gets

$$
\begin{gathered}
\operatorname{ad}_{\kappa(p)^{-1}}\left(\theta_{A^{s}}-\theta_{A^{0}}\right)(w)=\left(\theta_{A^{s}}-\theta_{A^{0}}\right)\left(\left[R_{\kappa(p)}\right]_{*}(w)\right)=\left(\theta_{A^{s}}-\theta_{A^{0}}\right)\left(k_{*}(w)\right)= \\
=-i \operatorname{pr}_{\mathfrak{k} \perp}\left[-\kappa(p)^{-1} d \kappa(J w)+i\left(\kappa(p)^{-1} d \kappa(w)\right]-\kappa(p)^{-1} d \kappa(w)=\right. \\
=-i \operatorname{pr}_{i \mathfrak{k}}\left[i\left(i \kappa(p)^{-1} d \kappa(J w)+\kappa(p)^{-1} d \kappa(w)\right)\right]-\kappa(p)^{-1} d \kappa(w)= \\
=-i \operatorname{pr}_{i \mathfrak{k}}\left[2 i \kappa(p)^{-1} \bar{\partial} \kappa(w)\right]-\kappa(p)^{-1} d \kappa(w)= \\
=-i \operatorname{pr}_{i \mathfrak{k}}\left[2 i \kappa(p)^{-1} \bar{\partial} \kappa(w)\right]-\kappa(p)^{-1} d \kappa(w)= \\
=-i\left[i \kappa(p)^{-1} \bar{\partial} \kappa(w)-i \partial \kappa(w) \kappa(p)^{-1}\right]-\kappa(p)^{-1} d \kappa(w)= \\
=-\partial \kappa(w) \kappa(p)^{-1}-\kappa(p)^{-1} \partial \kappa(w),
\end{gathered}
$$

so that

$$
\begin{gathered}
\left(\theta_{A^{s}}-\theta_{A^{0}}\right)(w)=\operatorname{ad}_{\kappa(p)}\left[-\partial \kappa(w) \kappa(p)^{-1}-\kappa(p)^{-1} \partial \kappa(w)\right]= \\
{\left[-\kappa(p) \partial \kappa(w) \kappa(p)^{-2}-\partial \kappa(w) \kappa(p)^{-1}\right]}
\end{gathered}
$$

But

$$
\chi^{-1} d \chi=\kappa^{2} \partial\left(\kappa^{-2}\right)=-\kappa(\partial \kappa) \kappa^{-2}-(\partial \kappa) \kappa^{-1} .
$$


Corollary 5.3 For the curvature of $A^{s}:=A_{e^{-\frac{s}{2}}\left(P^{0}\right), J}$ one gets the formula

$$
F_{A^{s}}=F_{A^{0}}+\bar{\partial}\left(h^{-1} \partial_{0} h\right)
$$

where $\bar{\partial}$ stands for the Dolbeault operator associated with the fixed holomorphic structure $J$ on $Q$.

Sometimes it is more convenient to work with connections on the fixed $K$ principal bundle $P^{0}$. Therefore, we will also consider the connection

$$
A_{s}:=\left[e^{-\frac{s}{2}}\right]^{*}\left(A^{s}\right) \in \mathcal{A}\left(P^{0}\right) .
$$

Proposition 5.4 The connection form and the curvature form of the connection $A_{s}$ are given by the formulae

$$
\begin{gathered}
A_{s}-A^{0}=k^{-1} \bar{\partial}_{0} k-\left(\partial_{0} k\right) k^{-1}=l^{-1}\left(\partial_{0} l\right)-\left(\bar{\partial}_{0} l\right) l^{-1}, \\
F_{A_{s}}=\operatorname{ad}_{l}\left(F_{A^{s}}\right)=\operatorname{ad}_{l}\left(F_{A^{0}}+\bar{\partial}\left(h^{-1} \partial_{0} h\right)\right),
\end{gathered}
$$

where $k:=e^{-s}$ and $l:=k^{-1}=\sqrt{h}$.

Proof:

$$
\begin{gathered}
A_{s}-A^{0}=k^{-1}\left(d_{A^{s}} k\right)+h^{-1} \partial_{0} h= \\
k^{-1}\left(d_{0} k\right)+k^{-1}\left[-k\left(\partial_{0} k\right) k^{-2}-\left(\partial_{0} k\right) k^{-1}, k\right]-k\left(\partial_{0} k\right) k^{-2}-\left(\partial_{0} k\right) k^{-1}= \\
=k^{-1}\left(d_{0} k\right)-\left(\partial_{0} k\right) k^{-1}-k^{-1}\left(\partial_{0} k\right)+k\left(\partial_{0} k\right) k^{-2}+\left(\partial_{0} k\right) k^{-1}-k\left(\partial_{0} k\right) k^{-2}-\left(\partial_{0} k\right) k^{-1} \\
=k^{-1}\left(\partial_{0} k\right)+k^{-1}\left(\bar{\partial}_{0} k\right)-\left(\partial_{0} k\right) k^{-1}-k^{-1}\left(\partial_{0} k\right)=k^{-1} \bar{\partial}_{0} k-\left(\partial_{0} k\right) k^{-1} .
\end{gathered}
$$

\subsection{The orbits of the adjoint action. Sections in the ad- joint bundle}

Let $K$ be a compact Lie group and $T \subset K$ a fixed maximal torus.

We need first to understand the structure of the quotient $\mathfrak{k} / K$, where $K$ acts on its Lie algebra $\mathfrak{k}$ via the adjoint representation.

First of all note that any element $\varphi \in \mathfrak{k}$ is conjugated to an element in $\mathfrak{t}$. Moreover, if two elements $\varphi_{1}, \varphi_{2} \in \mathfrak{t}$ are conjugated, i. e. $\operatorname{ad}_{k}\left(\varphi_{1}\right)=\varphi_{2}$ for some $k \in K$, then $\operatorname{ad}_{k}(T)$ and $T$ are both maximal tori of $K$ which are contained in the connected centralizer $Z_{K}\left(\varphi_{2}\right)$. Therefore, these tori are conjugated in $Z_{K}\left(\varphi_{2}\right)$, hence there exists $u \in Z_{K}\left(\varphi_{2}\right)$ such that $\operatorname{ad}_{u}\left(\operatorname{ad}_{k}(T)\right)=T$. This shows that $\kappa:=u k$ belongs to the normalizer $N_{K}(T)$ of $T$ in $K$, hence $\operatorname{ad}_{\kappa}\left(\varphi_{1}\right)=$ 
$\operatorname{ad}_{u}\left(\varphi_{2}\right)=\varphi_{2}$, so that $\varphi_{1}, \varphi_{2}$ are congruent modulo $N_{K}(T)$. Concluding, we get an identification

$$
\mathfrak{k} / K=\mathfrak{t} / N_{K}(T)=\mathfrak{t} / W_{K}(T) .
$$

When $K$ is connected the quotient $\mathfrak{t} / W_{K}(T)$ can be identified with any closed Weyl chamber $C \subset \mathfrak{t}$. Indeed, it suffices to note that two distinct elements on the boundary of $C$ cannot be congruent modulo $W_{K}(T)$.

To prove this, let $x, y \in C \backslash \dot{C}$ be two points which are congruent modulo $W_{K}(T)$. Let $w \in W_{K}(T)$ such that $w(x)=y$. Then $y \in C \cap w(C)$. Let $c$ be the common face of $C$ and $w(C)$ of minimal dimension which contains $y$.

Since the chambers $C$ and $w(C)$ have a common face $c$, one can find a sequence of chambers $\left(C_{i}\right)_{1 \leq i \leq k}$ such that $C_{1}=C, C_{k}=w(C)$ and $C_{i}, C_{i+1}$ have a common codimension 1 face which contains $c$. It follows that $C_{i}$ is mapped to $C_{i+1}$ by the reflexion with respect to the hyperplane $H_{i}$ generated by this common codimension 1 face. Therefore $C$ is mapped to $w(C)$ by a composition of reflexions with respect to hyperplanes which contain $c$. Since the operation of the Weyl group is free transitive on the set of chambers, it follows that $w$ coincides with this composition, hence it leaves $y$ fixed.

Therefore,

Remark 5.5 Suppose that $K$ is connected, and let $C \subset \mathfrak{t}$ be a closed Weyl chamber. The natural map

$$
C \longrightarrow \mathfrak{k} / K
$$

is a homeomorphism.

Let $K$ be a connected compact Lie group and $P$ a principal $K$-bundle over an arbitrary manifold $B$. We fix a closed Weyl chamber $C \subset \mathfrak{t}$. $C$ decomposes as a disjoint union of convex sets, the open faces of $C$. Each open face is the interior of a face of $C$ (in the linear subspace generated by this face).

Lemma 5.6 Let $\varphi \in \Gamma(X, \operatorname{ad}(P))$ be a section regarded as a $K$-equivariant map $P \rightarrow \mathfrak{k}$. Suppose that the map

$$
B \stackrel{[\varphi]}{\longrightarrow} \mathfrak{k} / K \stackrel{\simeq}{\longrightarrow} C
$$

takes values in a fixed open face $c$ of $C$.

Then:

1. The space $\varphi^{-1}(c) / T \subset P / T$ is a locally trivial fibre bundle with standard fibre $Z_{K}(c) / T$ over $B$. 
2. Let $A$ be any connection on $P$. The map $B \stackrel{[\varphi]}{\longrightarrow} \mathfrak{k} / K$ is locally constant if and only if the projection of $d_{A, x}(\varphi)$ on $z_{\mathfrak{k}_{x}}\left(\varphi_{x}\right) \subset \mathfrak{k}_{x}:=P_{x} \times_{\text {ad }} \mathfrak{k}$ vanishes for every $x \in B$.

Proof: 1. Note first that centralizer $Z_{K}(\gamma)$ of any element $\gamma \in c$ is the same, hence $Z_{K}(\gamma)=Z_{K}(c)$ for every $\gamma \in c$. Indeed, since $K$ is connected, any centralizer of the form $Z_{K}(u)$ with $u \in \mathfrak{k}$ is connected, so it suffices to look at the Lie algebras of these centralizers. But the Lie algebra $z_{k}(u)$ has the following simple description in terms of the root set $R \subset \operatorname{Hom}_{\mathbb{R}}(\mathfrak{t}, i \mathbb{R})$ :

$$
z_{k}(u)=\left[\bigoplus_{\alpha \in R, \alpha(u)=0} \mathfrak{k}_{\alpha}^{\mathbb{C}}\right] \cap \mathfrak{k} .
$$

Therefore it suffices to note that the map

$$
\gamma \mapsto\{\alpha \in R \mid \alpha(\gamma)=0\} \subset R
$$

is constant on the open face $c$.

The map

$$
K / Z_{K}(c) \times c \longrightarrow \mathfrak{k},([k], \gamma) \mapsto \operatorname{ad}_{k}(\gamma)
$$

is an embedding, so its image $\operatorname{ad}_{K}(c)$ is a submanifold of $\mathfrak{k}$.

The restriction of the map

$$
\varphi: P \rightarrow \operatorname{ad}_{K}(c)
$$

to any fibre $P_{x}$ is obviously a submersion, hence $\varphi$ is also a submersion. The manifold $\varphi^{-1}(c)$ is obviously a principal $Z_{K}(c)$-bundle over $B$. Therefore, the quotient $\varphi^{-1}(c) / T$ is just the associated bundle with fibre $Z_{K}(c) / T$.

2. Let $s \in \operatorname{ad}_{K}(c)$. The Lie algebra $\mathfrak{k}$ decomposes as

$$
\mathfrak{k}=z_{\mathfrak{k}}(s) \oplus[\mathfrak{k}, s],
$$

whereas the tangent space at $s$ of the submanifold $\operatorname{ad}_{K}(c) \subset \mathfrak{k}$ decomposes as

$$
T_{s}\left(\operatorname{ad}_{K}(c)\right)=[\mathfrak{k}, s] \oplus\left[z_{\mathfrak{k}}(s) \cap T_{s}\left(\operatorname{ad}_{K}(c)\right)\right]=T_{s}\left(\operatorname{ad}_{k}(s)\right) \oplus\left[z_{\mathfrak{k}}(s) \cap T_{s}\left(\operatorname{ad}_{K}(c)\right)\right] .
$$

Therefore, a map $\sigma: U \rightarrow c$ on a manifold $U$ defines a locally constant map $[\sigma]: U \rightarrow \mathfrak{k} / K$ if and only if $\operatorname{pr}_{z_{\mathfrak{k}}\left(\sigma_{x}\right)}\left(d_{x} \sigma\right)=0$ for all $x \in U$.

In our case, we deduce that the map $P \rightarrow \mathfrak{k} / K$ defined by $\varphi: P \rightarrow \mathfrak{k}$ is locally constant if and only if $\operatorname{pr}_{z_{\mathfrak{k}}\left(\varphi_{p}\right)}\left(d_{p} \varphi\right)=0$ for all $p \in P$. But, for a vertical tangent vector $v \in T_{p}(P)$ one obviously has $d_{p} \varphi(v) \in\left[\mathfrak{k}, \varphi_{x}\right]$, so the condition $\operatorname{pr}_{z_{\mathfrak{k}}\left(\varphi_{p}\right)}\left(d_{p} \varphi\right)=0$ is in fact equivalent with the condition $\operatorname{pr}_{z_{\mathfrak{k}_{x}}\left(\varphi_{x}\right)}\left(d_{A, x} \varphi\right)=0$. 
Lemma 5.7 Let $K$ be a maximal compact subgroup of the reductive group $G$. The natural map

$$
i \mathfrak{k} / K \longrightarrow \mathfrak{g} / / G
$$

is injective.

Here we denoted by $\mathfrak{g} / /{ }_{G}$ the GIT quotient of $\mathfrak{g}$ with respect to the adjoint action of $G$. In other words,

$$
\mathfrak{g} / / G=\operatorname{Spec}\left\{\mathbb{C}[\mathfrak{g}]^{G}\right\} .
$$

Proof: The moment map for the $K$-action on $\mathfrak{g}$ is

$$
m: \gamma \mapsto-\frac{i}{2}\left[\gamma, \gamma^{*}\right],
$$

where $(\cdot)^{*}$ is the conjugation with respect to the real structure $\mathfrak{g}=[i \mathfrak{k}] \otimes \mathbb{C}$.

It suffices to note that $\mathfrak{k}$ is contained in $m^{-1}(0)$.

Corollary 5.8 Let $\iota_{1}, \ldots, \iota_{k} \in \mathbb{C}[\mathfrak{g}]$ be homogeneous generators of the invariant algebra $\mathbb{C}[\mathfrak{g}]^{G}$. Then two elements $u, v \in i \mathfrak{k}$ are in the same $K$-orbit if and only if $\iota_{i}(u)=\iota_{i}(v)$ for $1 \leq i \leq k$.

In particular, a section $\sigma$ in the adjoint bundle $P \times_{\text {ad }}$ ik of a principal $K$-bundle is constant (respectively constant almost everywhere) if and only if the complex valued functions $\iota_{i}(\sigma)$ are constant (respectively constant almost everywhere) for $1 \leq i \leq k$.

The second statement in Lemma 5.6 holds for general possibly non-connected compact Lie groups. The converse statement is also true. More precisely, one has

Proposition 5.9 Let $K$ be a compact Lie group, $P$ a principal $K$-bundle, $A$ a connection on $P$ and $\varphi \in \Gamma(X, \operatorname{ad}(P))$ regarded as a $K$-equivariant map $P \rightarrow \mathfrak{k}$.

The map $B \stackrel{[\varphi]}{\longrightarrow} \mathfrak{k} / K$ defined by $\varphi$ is locally constant if and only if the projection $\operatorname{pr}_{z_{\mathfrak{k}_{x}}\left(\varphi_{x}\right)}\left(d_{A, x} \varphi\right)$ vanishes for every $x \in B$.

\section{Proof:}

We suppose that the base $B$ is connected.

1. The implication:

$$
[\varphi] \text { is constant } \Rightarrow\left[\operatorname{pr}_{z_{\mathfrak{k}_{x}}\left(\varphi_{x}\right)}\left(d_{A, x} \varphi\right)=0 \forall x \in B\right] .
$$


In the case when $K$ is connected, one just takes the open chamber $c$ which contains the conjugacy class of $[\varphi]$ and applies Lemma 5.6.

The general case can be reduced to the connected case by taking a finite covering of the base $B$ on which $P$ admits a $K_{e}$-reduction $P_{e} \subset P$. It suffices to note that, in general, a continuous map $U \longrightarrow \mathfrak{k} / K_{e}$ on a connected manifold $U$ is constant if and only if the induced map $U \longrightarrow \mathfrak{k} / K$ is constant.

\section{The implication:}

$$
\left[\operatorname{pr}_{z_{\mathfrak{k}_{x}}\left(\varphi_{x}\right)}\left(d_{A, x} \varphi\right)=0 \forall x \in B\right] \Rightarrow[\varphi] \text { is constant } .
$$

Let $j_{i}: \mathfrak{g}^{d_{i}} \rightarrow \mathbb{C}$ be the $\operatorname{ad}_{G}$-invariant symmetric multilinear map which corresponds to $\iota_{i}$ (see Corollary 5.8).

One has

$$
d\left[j_{i}(\varphi, \ldots, \varphi)\right]=d_{i} j_{i}\left(d_{A} \varphi, \varphi, \ldots, \varphi\right) .
$$

On the other hand, taking into account that $j_{i}$ is $\operatorname{ad}_{G}$-invariant, it follows easily that, for any $v \in \mathfrak{g}$, the linear functional $j_{i}(\cdot, v, \ldots, v)$ vanishes on $[v, \mathfrak{k}]$. The point is that, for $v \in \mathfrak{k}$, one has an orthogonal decomposition

$$
\mathfrak{k}=[v, \mathfrak{k}] \oplus z_{\mathfrak{k}}(v) .
$$

Therefore, one gets

$$
d\left[j_{i}(\varphi, \ldots, \varphi)\right]=d_{i} j_{i}\left(d_{A} \varphi, \varphi, \ldots, \varphi\right)=d_{i} j_{i}\left(\operatorname{pr}_{z_{\mathfrak{k}}(v)}\left(d_{A}(\varphi)\right), \varphi, \ldots, \varphi\right)=0 .
$$

The claim follows now from Corollary 5.8.

In section 1.2 we have used the following technical result:

Proposition 5.10 Let $K$ be a maximal compact subgroup of a complex reductive group $G$ and $s, \dot{s} \in i \mathfrak{k}$. Using the decomposition (68), write

$$
\dot{s}=\lambda+[k, s],
$$

with $\lambda \in i z_{\mathfrak{k}}(s), k \in z_{\mathfrak{k}}(s)^{\perp}$. Then

1.

$$
\left(d_{s} \exp \right)(\dot{s}) e^{-s}=\lambda+\left(k-\operatorname{ad}_{e^{s}} k\right) .
$$

2.

$$
\left\langle\dot{s}, p_{i \mathfrak{k}}\left\{\left(d_{s} \exp \right)(\dot{s}) e^{-s}\right\}\right\rangle \geq\|\dot{s}\|^{2}
$$


3.

$$
\left\langle\left[p_{\mathfrak{k}}\left\{\left(d_{s} \exp \right)(\dot{s}) e^{-s}\right\}, s\right], p_{i \mathfrak{k}}\left\{\left(d_{s} \exp \right)(\dot{s}) e^{-s}\right\}\right\rangle \leq 0
$$

with equality if and only if $\dot{s} \in i z_{\mathfrak{k}}(s)$.

\section{Proof:}

Put

$$
\dot{s}=\lambda+[k, s]=\lambda+\frac{d}{d t}\left(\operatorname{ad}_{e^{t k}}(s)\right), \lambda \in i z_{\mathfrak{k}}(s), k \in z_{\mathfrak{k}}(s)^{\perp} .
$$

One has

$$
\begin{gathered}
\left(d_{s} \exp \right)(\dot{s})=\left(d_{s} \exp \right)(\lambda)+\left(d_{s} \exp \right)([k, s])=\lambda e^{s}+\left.\frac{d}{d t}\right|_{t=0} \operatorname{Ad}_{e^{t k}} e^{s}= \\
{\left[\lambda+\left(k-\operatorname{ad}_{e^{s}} k\right)\right] e^{s},}
\end{gathered}
$$

so

$$
\sigma:=\left(d_{s} \exp \right)(\dot{s}) e^{-s}=\lambda+\left(k-\operatorname{ad}_{e^{s}} k\right) .
$$

Let $R_{s}$ denote the set of eigenvalues of the endomorphism $[s, \cdot]$ on $\mathfrak{g}$. Decompose

$$
k=\sum_{\rho \in R_{s} \backslash\{0\}} k_{\rho}, k_{\rho} \in \mathfrak{g}_{\rho},
$$

with $\bar{k}_{\rho}=-k_{-\rho}($ since $k \in \mathfrak{k})$. Then

$$
\begin{gathered}
{[s, k]=\sum_{\rho \in R_{s} \backslash\{0\}} \rho k_{\rho}, \operatorname{ad}_{e^{s}} k=\sum_{\rho \in R_{s} \backslash\{0\}} e^{\rho} k_{\rho},} \\
\dot{s}=\lambda-\sum_{\rho \in R_{s} \backslash\{0\}} \rho k_{\rho}, \sigma=\lambda+\sum_{\rho \in R_{s} \backslash\{0\}}\left(1-e^{\rho}\right) k_{\rho} .
\end{gathered}
$$

We get

$$
\begin{gathered}
p_{i \mathfrak{k}}\left(\operatorname{ad}_{e^{s}} k\right)=\frac{1}{2}\left(\operatorname{ad}_{e^{s}} k+\overline{\operatorname{ad}_{e^{s}} k}\right)=\frac{1}{2} \sum_{\rho \in R_{s} \backslash\{0\}}\left(e^{\rho}-e^{-\rho}\right) k_{\rho}, \\
p_{\mathfrak{k}}\left(\operatorname{ad}_{e^{s}} k\right)=\frac{1}{2}\left(\operatorname{ad}_{e^{s}} k-\overline{\operatorname{ad}_{e^{s}} k}\right)=\frac{1}{2} \sum_{\rho \in R_{s} \backslash\{0\}}\left(e^{\rho}+e^{-\rho}\right) k_{\rho}, \\
p_{\mathfrak{k}}\left(k-\operatorname{ad}_{e^{s}} k\right)=\frac{1}{2} \sum_{\rho \in R_{s} \backslash\{0\}}\left(2-e^{\rho}-e^{-\rho}\right) k_{\rho},
\end{gathered}
$$




$$
\begin{gathered}
{\left[p_{\mathfrak{k}}(\sigma), s\right]=\frac{1}{2} \sum_{\rho \in R_{s} \backslash\{0\}} \rho\left(e^{\rho}+e^{-\rho}-2\right) k_{\rho}=\frac{1}{2} \sum_{\rho \in R_{s} \backslash\{0\}} \rho e^{-\rho}\left(e^{\rho}-1\right)^{2} k_{\rho},} \\
p_{i \mathfrak{k}}(\sigma)=\lambda+\frac{1}{2} \sum_{\rho \in R_{s} \backslash\{0\}}\left(-e^{\rho}+e^{-\rho}\right) k_{\rho} .
\end{gathered}
$$

Therefore,

$$
\begin{gathered}
\left\langle\dot{s}, p_{i \mathfrak{k}}(\sigma)\right\rangle=\|\lambda\|^{2}+\frac{1}{2} \sum_{\rho \in R_{s} \backslash\{0\}} \rho\left(e^{\rho}-e^{-\rho}\right)\left\|k_{\rho}\right\|^{2} \geq \\
\geq\|\lambda\|^{2}+\sum_{\rho \in R_{s} \backslash\{0\}} \rho^{2}\left\|k_{\rho}\right\|^{2}=\|\dot{s}\|^{2},
\end{gathered}
$$

because the inequality $\left|e^{x}-e^{-x}\right| \geq 2|x|$ holds on $\mathbb{R}$.

For the third inequality we compute

$$
\begin{gathered}
\left\langle\left[p_{\mathfrak{k}}(\sigma), s\right], p_{i \mathfrak{k}}(\sigma)\right\rangle= \\
=-\sum_{\rho \in R_{s} \backslash\{0\}} \frac{1}{4} \rho\left(e^{\rho}-e^{-\rho}\right) e^{-\rho}\left(e^{\rho}-1\right)^{2}\left\|k_{\rho}\right\|^{2} \leq 0 .
\end{gathered}
$$

Equality occurs s if and only if $\sum_{\rho} \rho k_{\rho}=0$.

\subsection{Local maximal torus reductions of a $K$-bundle}

We quote from $[\mathrm{LT}]$ the following Lemma (see $[\mathrm{LT}]$, section 7.4)

Lemma 5.11 Let $X$ be a manifold, and

$$
X=G_{r} \supset G_{r-1} \supset \ldots \supset G_{1}
$$

a filtration by closed subsets. Define $F_{k}:=G_{k} \backslash G_{k-1}, k=2, \ldots, r$. Then

$$
W:=\bigcup_{k=2}^{r} \stackrel{\circ}{F}_{k}
$$

is open and dense in $X$.

Proposition 5.12 For any $f \in \Gamma(B, \operatorname{ad}(P))$ there is an open dense subset $W \subset X$ such that for every $x \in W$ there exists an open face $c_{x} \subset C$, an open neighbourhood $U_{x}$ of $x$ in $B$ and a T-reduction $\left.\Pi_{x} \subset P\right|_{U_{x}}$ of the restriction $\left.P\right|_{U_{x}}$ such that $\left.f\right|_{U_{x}}$ is defined by a smooth map

$$
\lambda \in \mathcal{C}^{\infty}\left(U_{x}, c_{x}\right) \subset \mathcal{C}^{\infty}\left(U_{x}, \mathfrak{t}\right)=\Gamma\left(U_{x}, \operatorname{ad}\left(\Pi_{x}\right)\right) .
$$




\section{Proof:}

Regard $f$ as a $K$-equivariant map $P \rightarrow \mathfrak{k}$, and let $[f]: B \rightarrow \mathfrak{k} / K=C$ be the induced $\mathfrak{k} / K$-valued map on $B$. Consider the skeleton filtration

$$
C=C_{d} \supset C_{d-1} \supset \ldots C_{1} \supset \ldots \supset C_{0}
$$

of $C$, where $C_{i}$ is the union of all open faces of dimension less or equal $i$. We get a filtration by closed sets

$$
B=B_{d} \supset B_{d-1} \supset \ldots B_{1} \supset \ldots \supset B_{0}
$$

of $B$, where $B_{i}:=[f]^{-1}\left(C_{i}\right)$.

Consider now the locally closed subsets

$$
F_{i}:=B_{i} \backslash B_{i-1}=[f]^{-1}\left(C_{i} \backslash C_{i-1}\right)=[f]^{-1}\left[\coprod_{\substack{c \subset C \text { open face } \\ \operatorname{dim}(c)=i}} c\right] .
$$

By Lemma 5.11, it follows that $W:=\bigcup \dot{F}_{i}$ is dense in $B$. For any $x \in W$ let $i_{x} \in\{0, \ldots, d\}$ such that $x \in{\stackrel{\circ}{F_{i x}}}_{i_{x}}$ and let $U_{x}$ be an arbitrary contractible open neighborhood of $x$ in ${\stackrel{\circ}{F_{x}}}_{i_{x}}$. Since $U_{x}$ is connected, $[f]\left(U_{x}\right)$ is contained in a single open face $c_{x}$ of $C$. Moreover, the fibre bundle $\left[\left.f\right|_{U_{x}} ^{-1}\left(c_{x}\right)\right] / T$ over the contractible manifold $U_{x}$ is trivial, hence one can find a section $\sigma_{x}$ in this bundle. Regarding $\sigma_{x}$ as a section in $\left[\left.P\right|_{U_{x}}\right] / T$, we get a $T$-reduction of $\left.P\right|_{U_{x}}$ on which $f$ is given by a smooth $c_{x}$-valued function.

Remark 5.13 Proposition 5.12 is also true for non-connected Lie groups $K$. The proof reduces to the connected case using a finite cover of $B$, on which $P$ can be reduced to the connected component $K_{e} \subset K$.

\subsection{Decomposing a connection with respect to a maximal torus reduction. Applications}

Let $P$ be a principal $K$-bundle over a manifold $B$ and let $\iota: \Pi \hookrightarrow P$ be a $T$-reduction of $P$. Consider the root set $R \subset \operatorname{Hom}(\mathfrak{t}, i \mathbb{R})$ and the root decomposition

$$
\mathfrak{g}=\bigoplus_{\rho \in R} \mathfrak{g}_{\rho}
$$

of $\mathfrak{g}:=\mathfrak{k}^{\mathbb{C}}$. 
Since this decomposition is $T$-invariant, and we have fixed a $T$-reduction of $P$, one gets a decomposition of the complexified adjoint bundle $\operatorname{ad}^{\mathbb{C}}(P)$ :

$$
\operatorname{ad}^{\mathbb{C}}(P)=\bigoplus_{\rho \in R} \Pi \times \text { ad } \mathfrak{g}_{\rho}
$$

Denote by $\mathfrak{t}^{\perp}$ the natural complement of the Lie algebra $\mathfrak{t}$ in $\mathfrak{k}$, i. e.

$$
\mathfrak{t}^{\perp}=\left[\bigoplus_{\rho \neq 0} \mathfrak{k}_{\rho}^{\mathbb{C}}\right] \cap \mathfrak{k} .
$$

Let $A$ be a connection on $P$, and $\omega_{A} \in A^{1}(P, \mathfrak{k})$ its connection form. Let $A_{\Pi}$ be the induced connection on $\Pi$, and $\iota_{*}\left(A_{\Pi}\right)$ the push-forward connection to $P$. Write

$$
\left.\omega_{A}=\omega_{\iota_{*}\left(A_{\Pi}\right.}\right)+a
$$

where $a \in A^{1}\left(\Pi \times_{\mathrm{ad}} \mathfrak{t}^{\perp}\right)$ is the second fundamental form of the subbundle $\Pi$ with respect to the connection $A$.

Suppose now that the basis $B$ is a complex manifold. Then one can write $a=a^{10}+a^{01}$, where each term decomposes as

$$
a^{10}=\sum_{\rho \neq 0} a_{\rho}^{10}, a_{\rho}^{10} \in A^{10}\left(\Pi \times_{\mathrm{ad}} \mathfrak{g}_{\rho}\right), a^{01}=\sum_{\rho \neq 0} a_{\rho}^{01}, a_{\rho}^{01} \in A^{01}\left(\Pi \times_{\mathrm{ad}} \mathfrak{g}_{\rho}\right) .
$$

With respect to the usual real structure $\mathfrak{g}=[i \mathfrak{k}]^{\mathbb{C}}$ of $\mathfrak{g}$, one has

$$
\overline{a_{\rho}^{10}}=-a_{-\rho}^{01} .
$$

Let $\varphi \in A^{0}(\operatorname{ad}(P))$ be a smooth section. Suppose now that, with respect to the reduction $\Pi$, the section $\varphi$ is induced by a smooth map $\lambda \in \mathcal{C}^{\infty}(B, c)$ where $c$ is a fixed open face of the Weyl chamber $C$. We know, by Proposition 5.12, that locally we can always come to this situation.

Via the decomposition (71), and taking into account that $[a, \lambda]=-[\lambda, a]$, we have the following formulae for $\partial_{A} \varphi, \bar{\partial}_{A} \varphi$ :

$$
\partial_{A} \varphi=\partial \lambda-\sum_{\rho \neq 0} \rho(\lambda) a_{\rho}^{10}, \bar{\partial}_{A} \varphi=\bar{\partial} \lambda-\sum_{\rho \neq 0} \rho(\lambda) a_{\rho}^{01}=\bar{\partial} \lambda+\sum_{\rho \neq 0} \rho(\lambda) \overline{a_{\rho}^{10}} .
$$

We will also need a formula for $e^{-s} \partial_{A}\left(e^{s}\right)$, where $s \in A^{0}(i \operatorname{ad}(P))$. Regard $s$ (and $e^{s}$ ) as an ad-equivariant (respectively Ad-equivariant) $\mathfrak{g}$-valued (respectively $G$-valued) function on $P$. Let be $x \in X, p \in \Pi_{x}$ and $v \in T_{x}(X)$ and $w \in T_{p}(\Pi)$ the $A_{\Pi}$ - horizontal lift of $v$. 
The $A$-horizontal component of $w$ is

$$
w^{A}=w-\left[\omega_{A}(w)\right]_{p}^{\#}=w-a(w)_{p}^{\#} .
$$

One has

$$
\begin{gathered}
e^{-s(p)} d\left(e^{s}\right)\left(w^{A}\right)=e^{-s(p)}\left[d\left(e^{s}\right)(w)-\left.\frac{d}{d t}\right|_{t=0}\left(e^{s(p \exp (t a(w))}\right)\right]= \\
=e^{-s(p)}\left[d\left(e^{s}\right)(w)-\left.\frac{d}{d t}\right|_{t=0}\left(e^{\operatorname{ad}(\exp (t a(w)))^{-1} s(p)}\right)\right]= \\
=e^{-s(p)}\left[d\left(e^{s}\right)(w)-\left.\frac{d}{d t}\right|_{t=0}\left(\operatorname{Ad}(\exp (t a(w)))^{-1}\left(e^{s(p)}\right)\right)\right]= \\
=e^{-s(p)}\left[d\left(e^{s}\right)(w)-\left[-a(w) e^{s(p)}+e^{s(p)} a(w)\right]\right] .
\end{gathered}
$$

Denote now by $\lambda$ the restriction $\lambda:=\left.s\right|_{\Pi}$. We get

$$
e^{-s(p)} d\left(e^{s}\right)\left(w^{A}\right)=d \lambda(w)+\sum_{\rho \neq 0}\left(e^{-\rho(\lambda)}-1\right) a_{\rho}(w),
$$

hence

$$
e^{-s} d_{A}\left(e^{s}\right)=d \lambda+\sum_{\rho \neq 0}\left(e^{-\rho(\lambda)}-1\right) a_{\rho},
$$

and similarly

$$
d_{A}\left(e^{s}\right) e^{-s}=d \lambda+\sum_{\rho \neq 0}\left(1-e^{\rho(\lambda)}\right) a_{\rho}
$$

In section 4.4.1 we need the following important technical results.

Let $E$ be a Hermitian space and $f: \mathbb{R} \rightarrow \mathbb{R}$ a real function. Using the spectral decomposition of Hermitian matrices, one can extend $f$ to a map (denoted by the same symbol to save on notations) $\operatorname{Herm}(E) \rightarrow \operatorname{Herm}(A)$ by putting

$$
f\left(\sum_{i} \lambda_{i} \operatorname{pr}_{F_{i}}\right):=\sum f\left(\lambda_{i}\right) \operatorname{pr}_{F_{i}}
$$

for every orthogonal direct sum decomposition of $E$.

It is known (see for instance $[\mathrm{Bh}]$ ) that

Proposition 5.14 The extension $\operatorname{Herm}(E) \rightarrow \operatorname{Herm}(E)$ of a continuous function $f: \mathbb{R} \rightarrow \mathbb{R}$ is continuous.

In general, this extension inherits the regularity properties of $f$. 
Remark 5.15 1. If $|f| \leq|g|$ then

$$
\|f(h)(v)\| \leq\|g(h)(v)\| \forall v \in E, \quad \forall h \in \operatorname{Herm}(E) .
$$

2. More generally, if on a subset $A \subset \mathbb{R}$ we have $\left.|f|_{A}|\leq| g\right|_{A} \mid$, then

$$
\|f(h)(v)\| \leq\|g(h)(v)\| \forall v \in E, \forall h \in \operatorname{Herm}(E) \text { with } \operatorname{Spec}(h) \subset A .
$$

Indeed, writing $h=\sum_{i} \lambda_{i} \operatorname{pr}_{F_{i}}$ as above, one gets

$$
\|f(h)(v)\|=\sqrt{\sum_{i} f\left(\lambda_{i}\right)^{2}\left\|\operatorname{pr}_{F_{i}}(v)\right\|^{2}} \leq \sqrt{\sum_{i} g\left(\lambda_{i}\right)^{2}\left\|\operatorname{pr}_{F_{i}}(v)\right\|^{2}}=\|g(h)(v)\| .
$$

Denote by $\eta: \mathbb{R} \rightarrow \mathbb{R}_{>0}$ the real analytic function defined by

$$
\eta(t)=\left\{\begin{array}{ccc}
\sqrt{\frac{1-e^{-t}}{t}} & \text { if } \quad t \neq 0 \\
1 & \text { if } t=0 .
\end{array}\right.
$$

Proposition 5.16 Let $\mathcal{Q}$ be a holomorphic principal $K^{\mathbb{C}}$-bundle on a complex manifold $X, P \subset \mathcal{Q}$ a K-reduction, denote by $A^{0}$ the corresponding Chern connection, and let $d_{0}=\bar{\partial}+\partial_{0}$ be the associated covariant derivative. Let $s \in A^{0}(P \times$ ad $i \mathfrak{k})$. Then one has

$$
\left.\left(i \Lambda_{g} \bar{\partial}\left(e^{-s} \partial_{0}\left(e^{s}\right)\right)\right), s\right)=\frac{1}{2} P\left(|s|^{2}\right)+\left|\eta([s, \cdot])\left(\partial_{0}(s)\right)\right|^{2} .
$$

Proof: Let $T$ be a maximal torus of $K$ and $R \subset \operatorname{Hom}(i t, \mathbb{R})$ be the root set. By Proposition 5.12 it suffices to check the formula in the case when $P$ has a $T$-reduction $\Pi \subset P$ and $s$ is given by a smooth function $\lambda: X \rightarrow i \mathfrak{t}$. By formulae (72) and (74) one has

$$
\partial_{0} s=\partial \lambda-\sum_{\rho \in R \backslash\{0\}} \rho(\lambda) a_{\rho}^{10}, e^{-s} \partial_{0}\left(e^{s}\right)=\partial \lambda+\sum_{\rho \in R \backslash\{0\}}\left(e^{-\rho(\lambda)}-1\right) a_{\rho}^{10} .
$$

Therefore, using the Hermitian inner product:

$$
\begin{gathered}
\left.\left(i \Lambda_{g} \bar{\partial}\left(e^{-s} \partial_{0}\left(e^{s}\right)\right)\right), s\right)=i \Lambda_{g} \bar{\partial}\left(e^{-s} \circ \partial_{0}\left(e^{s}\right), s\right)+i \Lambda_{g}\left(e^{-s} \circ \partial_{0}\left(e^{s}\right), \partial_{0} s\right)= \\
=i \Lambda_{g} \bar{\partial}(\partial \lambda, \lambda)+i \Lambda_{g}(\partial \lambda, \partial \lambda)-\sum_{\rho \in R \backslash\{0\}} \rho(\lambda)\left(e^{-\rho(\lambda)}-1\right) i \Lambda_{g}\left(a_{\rho}^{10}, a_{\rho}^{10}\right)= \\
(P(\lambda), \lambda)-\sum_{\rho \in R \backslash\{0\}} \rho(\lambda)\left(e^{-\rho(\lambda)}-1\right) i \Lambda_{g}\left(a_{\rho}^{10}, a_{\rho}^{10}\right) .
\end{gathered}
$$


On the other hand,

$$
\left.P\left(|s|^{2}\right)=i \Lambda_{g} \bar{\partial} \partial\left(|\lambda|^{2}\right)=2(P(\lambda), \lambda)-|\partial \lambda|^{2}\right) \leq 2(P(\lambda), \lambda) .
$$

Now it suffices to note that

$$
|\partial \lambda|^{2}-\sum_{\rho \in R \backslash\{0\}} \rho(\lambda)\left(e^{-\rho(\lambda)}-1\right) i \Lambda_{g}\left(a_{\rho}^{10}, a_{\rho}^{10}\right)=\left|\eta([s, \cdot])\left(\partial_{0}(s)\right)\right|^{2} .
$$

\subsection{Analytic results}

Lemma 5.17 Let $E, F$ be vector bundles with inner products on a measurable space $X$ with finite measure. Let $\left(u_{n}\right)_{n}$ be a sequence of $L^{2}$-sections on $E$ weakly convergent in $L^{2}$ to a section $u$, and let $\left(v_{n}\right)_{n}$ be a sequence of $L^{2}$-sections on $F$ strongly convergent in $L^{2}$ to a section $v$. Suppose that $v_{n}$ converges almost everywhere to $v$ and that $\left(\left|v_{n}\right|\right)_{n}$ is uniformly bounded by a positive constant $M$. Then it holds:

1. For every $L^{2}$-section $\psi$ in $(E \otimes F)^{\vee}$, the sequence $\left(v_{n} \psi\right)_{n}$ of sections in $E^{\vee}$ converges strongly in $L^{2}$ to $v \psi$.

2. The sequence $\left(u_{n} \otimes v_{n}\right)_{n}$ converges weakly in $L^{2}$ to $(u \otimes v)$.

Proof: 1. This follows from the Lebesgue dominated convergence theorem, since the sequence of non-negative functions $\left(\left|v_{n} \psi-v \psi\right|^{2}\right)_{n}$ converges almost everywhere to 0 and is bounded almost everywhere by the integrable function $4 M^{2}\left[\left.\psi\right|^{2}\right.$.

2. We have:

$$
\begin{gathered}
\left\langle u_{n} \otimes v_{n}, \psi\right\rangle_{L^{2}}-\langle u \otimes v, \psi\rangle_{L^{2}}=\left\langle u_{n}, v_{n} \psi\right\rangle_{L^{2}}-\langle u, v \psi\rangle_{L^{2}}= \\
=\left\langle u_{n}, v_{n} \psi-v \psi\right\rangle_{L^{2}}+\left\langle u_{n}-u, v \psi\right\rangle_{L^{2}} .
\end{gathered}
$$

The second term converges to 0 since, by hypothesis, $u_{n} \rightarrow u$ weakly. The first term tends to 0 because

$$
\left|\left\langle u_{n}, v_{n} \psi-v \psi\right\rangle_{L^{2}}\right| \leq\left\|u_{n}\right\|_{L^{2}}\left\|v_{n} \psi-v \psi\right\|_{L^{2}},
$$

$\left(u_{n}\right)_{n}$ is bounded 4 in $L^{2}$ and $\left\|v_{n} \psi-v \psi\right\|_{L^{2}} \rightarrow 0$ by 1 .

\footnotetext{
${ }^{4}$ Any weakly convergent sequence in a Hilbert space is bounded.
} 
Lemma 5.18 Let $D^{n_{i}} \subset \mathbb{R}^{n_{i}}$ be the standard $n_{i}$-dimensional disk, $i=1,2$, and let $\varphi \in L_{1}^{p}\left(D^{n_{1}} \times D^{n_{2}}\right), 1 \leq p<\infty$. Then for almost all $y \in D^{n_{2}}$ the following holds:

1. $\left.\varphi\right|_{D^{n_{1} \times\{y\}}}$ belongs to $L_{1}^{p}\left(D^{n_{1}}\right),\left.\partial_{x} \varphi\right|_{D^{n_{1} \times\{y\}}}$ belongs to $L^{p}\left(D^{n_{1}}\right)$.

2. $d\left(\left.\varphi\right|_{D^{n_{1}} \times\{y\}}\right)=\left.\left(\partial_{x} \varphi\right)\right|_{D^{n_{1}} \times\{y\}}$ in $L^{p}\left(D^{n_{1}}\right)$.

Proof: By Fubini's theorem $\left.\varphi\right|_{D^{n_{1}} \times\{y\}}$ and $\left.d \varphi\right|_{D^{n_{1}} \times\{y\}}$ belong to $L^{p}\left(D^{n_{1}}\right)$ for almost all $y \in D^{n_{2}}$. In particular, $\left.\partial_{x} \varphi\right|_{D^{n_{1}} \times\{y\}}$ belongs to $L^{p}\left(D^{n_{1}}\right)$ for almost all $y \in D^{n_{2}}$, too.

Let $\varphi_{n}$ be a sequence of smooth functions converging to $\varphi$ in the $L_{1}^{p}$ - topology as $n \rightarrow \infty$. Therefore

$$
\int_{D^{n_{1}} \times D^{n_{2}}}\left|\varphi_{n}-\varphi\right|^{p} \rightarrow 0, \int_{D^{n_{1}} \times D^{n_{2}}}\left|d \varphi_{n}-d \varphi\right|^{p} \rightarrow 0 .
$$

In particular,

$$
\int_{D^{n_{1}} \times D^{n_{2}}}\left|\partial_{x} \varphi_{n}-\partial_{x} \varphi\right|^{p} \rightarrow 0 .
$$

By Fubini's theorem, it follows that the positive functions

$$
y \mapsto \int_{D^{n_{1} \times\{y\}}}\left|\varphi_{n}-\varphi\right|^{p}, y \mapsto \int_{D^{n_{1} \times\{y\}}}\left|\partial_{x} \varphi_{n}-\partial_{x} \varphi\right|^{p}
$$

converge to 0 with respect to the $L^{1}$ - norm, so (taking a subsequence if necessary) one can assume that they converge to 0 almost everywhere on $D^{n_{2}}$. Therefore, for almost every $y \in D^{n_{2}}$, one has

$$
\left.\left.\varphi_{n}\right|_{D^{n_{1}} \times\{y\}} \stackrel{L^{p}}{\longrightarrow} \varphi\right|_{D^{n_{1} \times\{y\}}},\left.\left.\left(\partial_{x} \varphi_{n}\right)\right|_{D^{n_{1}} \times\{y\}} \stackrel{L^{p}}{\longrightarrow}\left(\partial_{x} \varphi\right)\right|_{D^{n_{1}} \times\{y\}} .
$$

But, since the $\varphi_{n}$ are smooth, one has

$$
\left.\left(\partial_{x} \varphi_{n}\right)\right|_{D^{n_{1} \times\{y\}}}=\partial_{x}\left[\left.\varphi_{n}\right|_{D^{n_{1}} \times\{y\}}\right] .
$$

One the other hand,

$$
\partial_{x}\left[\left.\varphi_{n}\right|_{D^{n_{1} \times\{y\}}}\right] \longrightarrow \partial_{x}\left[\left.\varphi\right|_{D^{n_{1} \times\{y\}}}\right] \text { as distribution }
$$

(because the distribution limit of $\left.\varphi_{n}\right|_{D^{n_{1} \times\{y\}}}$ is $\left.\varphi\right|_{D^{n_{1} \times\{y\}}}$ ). Therefore,

$$
\begin{gathered}
\left.\left.\varphi_{n}\right|_{D^{n_{1}} \times\{y\}} \stackrel{L^{p}}{\longrightarrow} \varphi\right|_{D^{n_{1}} \times\{y\}}, \partial_{x}\left[\left.\varphi_{n}\right|_{D^{n_{1} \times\{y\}}}\right] \stackrel{L^{p}}{\longrightarrow} \partial_{x}\left[\left.\varphi\right|_{D^{n_{1} \times\{y\}}}\right]= \\
=\left.\left(\partial_{x} \varphi\right)\right|_{D^{n_{1}} \times\{y\}},
\end{gathered}
$$

which shows that 1 . and 2 . hold for such $y$. 


\subsection{Meromorphic parabolic reductions defined by weakly holomorphic $L_{1}^{2}$ - sections}

Let $M$ be a complex manifold. We begin with the following definition:

Definition 5.19 A measurable map $F: B^{n} \rightarrow M$ is called "holomorphic on almost all lines" if

1. $n=1, F$ coincides almost everywhere with a holomorphic map $B \rightarrow M$.

2. $n>1$, and for any holomorphic parameterization $D \times \Delta \stackrel{\varphi}{\rightarrow} \cup \subset B^{n}$ with $D \subset \mathbb{C}, \Delta \subset \mathbb{C}^{n-1}$ the restriction $F \circ \varphi(\cdot, \zeta): D \rightarrow M$ is weakly holomorphic in the sense of 1., for almost all $\zeta \in \Delta$.

We recall the following fundamental results (see Shiffman [Sh1], UhlenbeckYau [UY1], [UY2]):

Theorem 5.20 Any measurable map $F: B^{n} \rightarrow M$ from the ball $B^{n} \subset \mathbb{C}^{n}$ into a projective algebraic manifold $M$, which is holomorphic on almost all lines, coincides almost everywhere with a meromorphic map.

Theorem 5.20 is used combined with an important regularity result (see UhlenbeckYau [UY1], [UY2]) which applies to the case $n=1$.

In order to state this result we need a brief preparation:

Let $Y$ be a compact manifold (possibly with boundary) and $N$ a closed submanifold of $\mathbb{R}^{m}$. We put

$$
L_{1}^{2}(Y, N):=\left\{\varphi \in L_{1}^{2}\left(Y, \mathbb{R}^{m}\right) \mid \varphi(y) \in N \text { for almost every } y \in Y\right\},
$$

and we endow this set with the topology induced from $L_{1}^{2}\left(Y, \mathbb{R}^{m}\right)$. The space $L_{1}^{2}(Y, N)$ obviously depends on the embedding $Y \hookrightarrow \mathbb{R}^{m}$. When $Y$ is a surface, one has the following important density property (see Schoen-Uhlenbeck [SU]).

Proposition 5.21 Suppose that $Y$ is a compact surface with possibly empty $\mathcal{C}^{1}$-boundary. Then $\mathcal{C}^{\infty}(Y, N)$ is dense in $L_{1}^{2}(Y, N)$.

This result has the following subtle consequence:

Corollary 5.22 Suppose that $Y$ is a compact surface with possibly empty $\mathcal{C}^{1}$ boundary and let $\varphi \in L_{1}^{2}(Y, M)$. Then $d \varphi\left(T_{y}(Y)\right) \subset T_{\varphi(y)}(M)$ for almost all $y \in Y$. 
Proof: By the density property Proposition 5.21, one can find a sequence $\varphi_{j} \in \mathcal{C}^{\infty}(Y, N)$ which converges in the $L_{1}^{2}\left(Y, \mathbb{R}^{m}\right)$ - topology to $\varphi$. There exists a subsequence $\left(\varphi_{j_{k}}\right)_{k}$ such that $\varphi_{j_{k}} \rightarrow \varphi$ and $d \varphi_{j_{k}} \rightarrow d \varphi$ almost everywhere as $k \rightarrow \infty$. It suffices to note that $T(N)$ is closed in $N \times \mathbb{R}^{m}$.

Now let $N \subset \mathbb{R}^{m}$ be a compact complex manifold embedded as a real submanifold in $\mathbb{R}^{m}$, and let $J_{N}$ be the corresponding almost complex structure. The inclusion $T_{x}(N) \hookrightarrow \mathbb{R}^{m}$ of the tangent space at $x \in Y$ induces an embedding

$$
T_{x}^{10}(N) \oplus T_{x}^{01}(N)=T_{x}^{\mathbb{C}}(N) \hookrightarrow \mathbb{C}^{m} .
$$

Therefore, one gets a filtration

$$
0 \subset T^{10}(N) \subset T^{\mathbb{C}}(N) \subset N \times \mathbb{C}^{m}
$$

of the trivial complex rank $m$ bundle over $N$.

Let $p: N \times \mathbb{C}^{m} \rightarrow T^{10}(N)$ be any bundle projection which induces the standard projection

$$
T^{\mathbb{C}}(N) \longrightarrow T^{10}(N), v \mapsto \frac{1}{2}\left(v-i J_{N}(v)\right)
$$

on $T^{\mathbb{C}}(N)$.

Remark 5.23 In the special case when the induced Riemannian metric on the submanifold $N \subset \mathbb{R}^{m}$ is Hermitian, one can just take the orthogonal projection with respect to the standard Hermitian structure on $N \times \mathbb{C}^{m}$ because, in this case, the direct sum $T^{10}(N) \oplus T^{01}(N)$ will be orthogonal with respect to this standard Hermitian structure.

We can now state

Theorem 5.24 [UY1], [UY2] Let $B \subset \mathbb{C}$ be the standard ball and let $N$ be a compact Kähler manifold embedded as a real submanifold in $\mathbb{R}^{N}$. Let $f: B \rightarrow N$ be a map with the following properties:

1. $f \in L_{1}^{2}(B, N)$.

2. $f$ is weakly holomorphic, i. e. one of the following two equivalent conditions holds:

(a) For almost all $b \in B$ the tangent map $T_{b}(B) \rightarrow T_{f(b)}(N)$ is $\mathbb{C}$-linear.

(b) For almost all $b \in B$ it holds $p_{f(b)} \circ\left(\bar{\partial}_{b} f\right)=0$, where $\bar{\partial} f$ stands for the $\bar{\partial}$-derivative $T_{b}^{01}(B) \rightarrow \mathbb{C}^{m}$ of $f: B \rightarrow \mathbb{C}^{m}$. 
Then $f$ coincides almost everywhere with a holomorphic map.

Combining Theorem 5.20 with the regularity Theorem 5.24, one gets the following important

Theorem 5.25 Let $M \subset \mathbb{R}^{m}$ be a complex projective algebraic manifold embedded as a real submanifold of $\mathbb{R}^{m}$, and let $p: M \times \mathbb{C}^{m} \rightarrow T^{10}(M)$ be a bundle projection which agrees with the standard projection $T^{\mathbb{C}}(M) \rightarrow T^{10}(M)$ on the subbundle $T^{\mathbb{C}}(M) \subset M \times \mathbb{C}^{m}$. Then any map $f \in L_{1}^{2}\left(B^{n}, M\right)$ satisfying the equation

$$
p \circ \bar{\partial} f=0
$$

almost everywhere on $B^{n}$, coincides almost everywhere with a meromorphic map.

Proof: This follows easily from the Theorem 5.20, the regularity Theorem 5.24 and Lemma 5.18.

Let $K$ be a maximal compact subgroup of the reductive group $G$. We will apply these results to an algebraic manifold of the form

$$
M=G / G\left(s_{0}\right)
$$

where $G\left(s_{0}\right)$ is the parabolic subgroup defined by an element $s_{0} \in i \mathfrak{k}$. We will need the following simple result.

Proposition 5.26 i) The map

$$
\operatorname{ad}_{K}\left(s_{0}\right)=K / Z_{K}\left(s_{0}\right) \rightarrow G / G\left(s_{0}\right)
$$

is a diffeomorphism.

ii) Endow $i \mathfrak{k}$ with an $\operatorname{ad}_{K}$-invariant inner product and consider the embedding $M=\operatorname{ad}_{K}\left(s_{0}\right) \hookrightarrow i \mathfrak{k}$ of the projective algebraic manifold $M$ in the Euclidian space $i \mathfrak{k}$ defined by the identification $M=\operatorname{ad}_{K}\left(s_{0}\right)$ in i). Then

1. For any $s \in \operatorname{ad}_{K}\left(s_{0}\right)=M$, the images of the morphisms

$$
T_{s}^{10}(M) \rightarrow(i \mathfrak{k}) \otimes \mathbb{C}=\mathfrak{g}, T_{s}^{01}(M) \rightarrow(i \mathfrak{k}) \otimes \mathbb{C}=\mathfrak{g}
$$

induced by this embedding are $\mathfrak{g}_{s}^{ \pm}$, where

$$
\mathfrak{g}_{s}^{ \pm}:=\bigoplus_{ \pm \lambda>0} \operatorname{Eig}([s, \cdot], \lambda)
$$


2. The induced Riemannian metric on $M$ is Kähler and homogeneous.

Proof: i) We just have to prove that the natural map

$$
K / Z_{K}\left(s_{0}\right) \longrightarrow G / G\left(s_{0}\right)
$$

is bijective. For surjectivity, let $g \in G$. We can decompose $g$ as $g=k u$, where $u \in \exp (i \mathfrak{k})$. Let $B \subset G\left(s_{0}\right) \cap G_{e}$ be a Borel subgroup contained in the parabolic subgroup $G\left(s_{0}\right) \cap G_{e}$ of the connected reductive group $G_{e}$ (the connected component of $e$ in $G)$. Since $\exp (i \mathfrak{k}) \in G_{e}$, one can further decompose $u=l b$, where $l \in K_{e}$ and $b \in B$, so one gets $g=(k l) b$, which shows that $g \equiv k l$ $\bmod G\left(s_{0}\right)$.

For injectivity, it suffices to show that

$$
K \cap G\left(s_{0}\right)=Z_{K}\left(s_{0}\right) .
$$

The inclusion $Z_{K}\left(s_{0}\right) \subset K \cap G\left(s_{0}\right)$ is obvious. The opposite inclusion follows by choosing an embedding $G \hookrightarrow G L(n, \mathbb{C})$ which maps $K$ into $U(n)$. Let $\Phi$ be the filtration of $\mathbb{C}^{n}$ defined by the eigenspaces of the Hermitian matrix $s_{0}$. More precisely, the $k$-term of this filtration is the direct sum of the eigenspaces corresponding to the first $k$ eigenvalues of $s_{0}$. It is well known that the parabolic subgroup $G L(n, \mathbb{C})\left(s_{0}\right) \subset G L(n, \mathbb{C})$ is just the stabilizer of the filtration $\Phi$. On the other hand, one has $G\left(s_{0}\right)=G \cap G L(n, \mathbb{C})\left(s_{0}\right)$. Let $g \in K \cap G\left(s_{0}\right)$. The matrix defined by $g$ is unitary and leaves invariant the filtration $\Phi$, so it leaves invariant all the eigenspaces of $s_{0}$ in $\mathbb{C}^{n}$. Therefore $g$ commutes with $s_{0}$.

ii)

1. Let $s=\operatorname{ad}_{k}\left(s_{0}\right) \in \operatorname{ad}_{K}\left(s_{0}\right)$. The tangent space $T_{s}\left(\operatorname{ad}_{K}\left(s_{0}\right)\right)$ is just the subspace $\operatorname{ad}_{k}\left(\left[\mathfrak{k}, s_{0}\right]\right)=[\mathfrak{k}, s] \subset i \mathfrak{k}$. The point which corresponds to $s$ via our identification $\operatorname{ad}_{K}\left(s_{0}\right)=G / G\left(s_{0}\right)$ is the congruence class $c:=k G\left(s_{0}\right) \in G / G\left(s_{0}\right)$. The tangent space of $G / G\left(s_{0}\right)$ at $c$ is

$$
T_{c}\left(G / G\left(s_{0}\right)\right)=\left(L_{k}\right)_{*}(\mathfrak{g}) /\left(L_{k}\right)_{*}\left(\mathfrak{g}\left(s_{0}\right)\right) .
$$

Now note that $\mathfrak{g}_{s_{0}}^{+}$is a complement of $\mathfrak{g}\left(s_{0}\right)$ in $\mathfrak{g}$, so one gets a natural isomorphism $T_{c}\left(G / G\left(s_{0}\right)\right)=\left(L_{k}\right)_{*}\left(\mathfrak{g}_{s_{0}}^{+}\right)=\left(R_{k}\right)_{*}\left(\mathfrak{g}_{s}^{+}\right)$.

The isomorphism $T_{s}\left(\operatorname{ad}_{K}\left(s_{0}\right)\right) \simeq T_{c}\left(G / G\left(s_{0}\right)\right)$ induced by our identification is given by

$$
[\mathfrak{k}, s] \longrightarrow \mathfrak{g}_{s}^{+} \stackrel{\left(R_{k}\right)_{*}}{\longrightarrow}\left(R_{k}\right)_{*}\left(\mathfrak{g}_{s}^{+}\right),
$$

where the first arrow is just the restriction $\rho_{s}:[\mathfrak{k}, s] \rightarrow \mathfrak{g}_{s}^{+}$of the projection $i \mathfrak{k} \rightarrow \mathfrak{g}_{s}^{+}$. The complex structure on the tangent space $[\mathfrak{k}, s]$ is the pull-back of the natural complex structure of $\mathfrak{g}_{s}^{+}$via $\rho_{s}$. Therefore, the subspaces $T_{s}^{10}\left(\operatorname{ad}_{K}\left(s_{0}\right)\right)$, 
$T_{s}^{01}\left(\operatorname{ad}_{K}\left(s_{0}\right)\right)$ of the complexified tangent space $T_{s}^{\mathbb{C}}\left(\operatorname{ad}_{K}\left(s_{0}\right)\right)=[\mathfrak{g}, s]=\mathfrak{g}_{s}^{+} \oplus \mathfrak{g}_{s}^{-}$ are just $\mathfrak{g}_{s}^{+}, \mathfrak{g}_{s}^{-}$.

2. It is easy to see that the Riemannian metric induced by the embedding $\operatorname{ad}_{K}\left(s_{0}\right)$ is Hermitian and homogeneous, i. e. invariant under the natural $K$ action. The fact that the corresponding Kähler metric $\omega$ is closed follows from the identity

$$
L_{X} \omega=d \iota_{X} \omega+\iota_{X} d \omega
$$

applied to the fundamental vector fields associated with the transitive $K$-action on $\operatorname{ad}_{K}\left(s_{0}\right)$.

Applying Theorem 5.25, we get

Proposition 5.27 Let $\varphi \in L_{1}^{2}\left(B^{n}, i \mathfrak{k}\right)$ such that $\varphi(x) \in \operatorname{ad}_{K}\left(s_{0}\right)$ for almost every $x \in B^{n}$. Suppose that

$$
\operatorname{pr}_{\mathfrak{g}_{\varphi(x)}^{+}}\left(\bar{\partial}_{x} \varphi\right)=0
$$

for almost every $x \in B^{n}$. Then $\varphi$ coincides almost everywhere with a meromorphic map $B^{n} \rightarrow M=\operatorname{ad}_{K}\left(s_{0}\right)$.

More generally, let $\mathcal{Q}$ be a holomorphic $G$-bundle on a complex manifold $X$, let $P \subset \mathcal{Q}$ be a K-reduction, and let $\sigma \in L_{1}^{2}\left(P \times_{\text {ad }} i \mathfrak{k}\right)$ be an $L_{1}^{2}$ section which defines an almost everywhere constant map $[\sigma]: X \rightarrow i \mathfrak{k} / K$ such that

$$
\operatorname{pr}_{V_{\sigma(x)}^{+}}\left(\bar{\partial}_{x} \sigma\right)=0
$$

for almost every $x \in X$. Fix a representative $\sigma_{0}$ in the conjugacy class defined by $\sigma$. Then $\sigma$ defines a meromorphic reduction of $\mathcal{Q}$ to $G\left(\sigma_{0}\right)$.

In the regular case, the holomorphic map $B^{n} \rightarrow G / G\left(s_{0}\right)$ associated with a smooth map $\varphi: B^{n} \rightarrow \operatorname{ad}_{K}\left(s_{0}\right)$ satisfying the holomorphy condition

$$
\operatorname{pr}_{V_{\sigma(x)}^{+}}\left(\bar{\partial}_{x} \sigma\right)=0 \forall x \in B^{n}
$$

is given explicitely by the formulae

$$
x \mapsto \text { the class mod } G\left(s_{0}\right) \text { of an element } k \in K \text { with } \operatorname{ad}_{k}\left(s_{0}\right)=\varphi(x),
$$

$$
x \mapsto\left\{g \in G \mid \operatorname{ad}_{g^{-1}}(\varphi(x)) \in \mathfrak{g}\left(s_{0}\right)\right\} .
$$

Similarly, the $G\left(s_{0}\right)$-reduction defined by a regular section $\sigma \in A^{0}\left(P \times_{\text {ad }} i \mathfrak{k}\right)$ satisfying our weak holomorphy condition is

$$
\mathcal{Q}(\sigma)=\left\{q \in \mathcal{Q} \mid \sigma(q) \in \mathfrak{g}\left(\sigma_{0}\right)\right\} .
$$

The converse of Proposition 5.27 is also true; more precisely 
Proposition 5.28 Let $\rho \subset \mathcal{Q} / G\left(\sigma_{0}\right)$ be a meromorphic $G\left(\sigma_{0}\right)$-reduction of a holomorphic principal $G$-bundle $\mathcal{Q}$ on a compact complex manifold $X$, and let $P \subset \mathcal{Q}$ be any $K$-reduction.

Then the section $\sigma$ associated with $\sigma_{0}$ via the $K \cap G\left(\sigma_{0}\right)=Z_{K}\left(\sigma_{0}\right)$-reduction $P \cap \mathcal{Q}^{\rho}$ of $\left.P\right|_{X_{\rho}}$ defines an element in $L_{1}^{2}\left(P \times_{\text {ad }} i \mathfrak{k}\right)$ which satisfies the weak holomorphy condition (81) and whose associated meromorphic $G\left(\sigma_{0}\right)$-reduction is $\rho$.

Proof: The crucial point here is the fact that $\sigma \in L_{1}^{2}\left(P \times{ }_{\text {ad }} i \mathfrak{k}\right)$. Let $A$ be the Chern connection of $P$. We make use of the formula (22) which was obtained in the proof of Theorem 3.3.

$$
\left.\int_{X_{\rho}}\left\langle i \Lambda_{g} F_{A}, \sigma\right)\right\rangle \operatorname{vol}_{g}=\frac{2 \pi}{(n-1) !} \operatorname{deg}(\rho, h(\xi))-\sum_{\lambda \in \operatorname{Spec}^{+}[\xi, \cdot]} \lambda\left\|a_{\lambda}\right\|_{L^{2}}^{2} .
$$

On the other hand, by formula (61), one has on $X_{\rho}$

$$
\partial_{0}\left(\left.\sigma\right|_{X_{\rho}}\right)=\sum_{\lambda \in \operatorname{Spec}^{+}\left[\sigma_{0}, \cdot\right]}\left[a_{\lambda},\left(\left.\sigma\right|_{X_{\rho}}\right)\right]=-\sum_{\lambda \in \operatorname{Spec}^{+}\left[\sigma_{0}, \cdot\right]} \lambda a_{\lambda}
$$

Comparing these formulae, one gets immediately

$$
\left\|\partial_{0}\left(\left.\sigma\right|_{X_{\rho}}\right)\right\|_{L^{2}}<\infty
$$

On the other hand, one can easily check that the form $\partial_{0}\left(\left.\sigma\right|_{X_{\rho}}\right)$ ), regarded as an $L^{2}$ - form on the whole manifold $X$, is in fact the distribution $\partial_{0}$ - derivative of the $L^{\infty}$ - section $\sigma$. The proof uses the same argument as for the analogous result for orthogonal projections on subsheaves of Hermitian holomorphic bundles.

\section{References}

[AG] Alvarez-Consul, L.; Garcia-Prada, O.: Hitchin-Kobayashi correspondence, quivers, and vortices, Comm. Math. Phys. 238, no. 1-2, 1-33, 2003.

[Au] Aubin, T.: Nonlinear Analysis on Manifolds, Monge-Ampere Equations, Springer-Verlag, New York, 1985.

[Bh] Bhatia, R.: Matrix Analysis, Springer-Verlag, New York, 1997.

[Ba] Banfield, D.: The geometry of coupled equations in gauge theory, D. Phil. Thesis, University of Oxford, 1996. 
[Bi] Biquard, O.: Les équations de Seiberg-Witten sur une surface complexe non kählerienne, Comm. Anal. Geom. 6, no. 1, 173-197, 1998.

[Bra] Bradlow, S. B.: Special metrics and stability for holomorphic bundles with global sections, J. Diff. Geom. 33, 169-214, 1991.

[BGM] Bradlow, S. B.; Garcia-Prada, O.; Mundet i Riera, I.: Relative HitchinKobayashi correspondences for principal pairs Q. J. Math. 54, no. 2, 171-208, 2003.

[Bru] Bruasse, L.: Harder-Narasimhan filtration on non Kähler manifolds, Int. Journal of Math., vol. 12, no 5, 579-594, 2001.

[BT] Bruasse, L.; Teleman, A.: Optimal one-parameter subgroups in Kähler geometry and gauge theory, preprint, IML, june 2003.

[Bu] Buchdahl, N.: Hermitian Einstein connections and stable vector bundles over compact complex surfaces, Math. Ann. 280, 625-648, 1988.

[Do1] Donaldson, S. K.: Anti self-dual connections over complex algebraic surfaces and stable vector bundles, Proc. London Math. Soc. 50, 1-26, 1985.

[Do2] Donaldson, S. K.: Infinite determinants, stable bundles and curvature, Duke Math. J. 54, 231-247, 1987.

[DK] Donaldson, S.; Kronheimer, P.: The Geometry of Four-Manifolds, Oxford Univ. Press, 1990.

[Dü] Dürr, M. B.: Seiberg-Witten theory and the $\mathcal{C}^{\infty}$-classification of complex surfaces, Ph. D. Thesis, Zürich University, 2002.

[Ga] Gauduchon, P.: Sur la 1-forme de torsion d'une variété hermitienne compacte, Math. Ann. 267, 495-518, 1984.

[HH] Heinzner, P.; Huckleberry, A.: Analytic Hilbert quotients, MSRI Publications, vol. 37, 309-349, 1999.

[Hi] Hitchin, N. J.: The self-duality equations on a Riemann surface, Proc. London Math. Soc. (3) 55, no. 1, 59-126, 1987.

[HL] Huybrechts, D.; Lehn, M.: Stable pairs on curves and surfaces, J. Alg. Geom. Vol. 4, 67-104, 1995.

[Ki] Kirwan, F. C.: Cohomology of quotients in symplectic and algebraic geometry, Math. Notes, Princeton University Press, 1984.

[Ko1] Kobayashi, S.: Curvature and stability of vector bundles, Proc. Jap. Acad. 58, 158-162, 1982. 
[Ko2] Kobayashi, S.: Differential geometry of complex vector bundles., Princeton Univ. Press, 1987.

[LY] Li, J.; Yau, S. T.: Hermitian Yang-Mills connections on non-Kähler manifolds, in Math. aspects of string theory (S.-T. Yau ed.), World Scient. Publ., 1987.

[Lin] Lin, T. R.: Hermitian-Yang-Mills-Higgs Metrics and stability for holomorphic vector bundles with Higgs Fields, Preprint, Rutgers University, New Brunswick.

[LL] Lübke, M.; Lupaşcu, P.: Isomorphy of the gauge theoretical and the deformation theoretical moduli space of simple holomorphic pairs, preprint, 2001.

[LO] Lübke, M.; Okonek, Ch.: Moduli spaces of simple bundles and Hermitian-Einstein connections, Math. Ann. Vol. 276, 663-674, 1987.

[LT] Lübke, M.; Teleman, A.: The Kobayashi-Hitchin correspondence, World Scientific Publishing Co., 1995.

[LYZ] Li, J; Yau, S. T.; Zheng, F.: On projectively flat Hermitian manifolds, Comm. in Analysis and Geometry, Vol. 2, 103-109, 1994.

[MS] Mehta, V.B.; Subramanian, S.: On the Harder-Narasimhan filtration of principal bundles, in Algebra, Arithmetic and Geometry (R. Parimpande ed.), 405-415, Narosa Pub. House, 2002.

[MFK] Mumford, D.; Fogarty; J., Kirwan, F.: Geometric invariant theory, Springer-Verlag, 1982.

[Mu1] Mundet i Riera, I.: A Hitchin-Kobayashi correspondence for Kaehler fibrations, J. Reine Angew. Math. 528, 41-80, 2000.

[Mu2] Mundet i Riera, I.: Hamiltonian Gromov-Witten invariants, prep. math. SG/0002121.

[OST] Okonek, Ch.; Schmitt, A.; Teleman, A.: Master spaces for stable pairs, Topology Vol. 38, No 1, 117-139, 1998.

[OT1] Okonek, Ch.; Teleman, A.: The Coupled Seiberg-Witten Equations, Vortices, and Moduli Spaces of Stable Pairs, Int. J. Math. Vol. 6 , No. 6, 893-910, 1995.

[OT2] Okonek, Ch.; Teleman, A.: Quaternionic monopoles, Common. Math. Phys. 180 (2), 363-388, 1996. 
[OT3] Okonek, Ch.; Teleman, A.: Gauge theoretical equivariant GromovWitten invariants and the full Seiberg-Witten invariants of ruled surfaces, Comm. Math. Phys. 227, no. 3, 551-585, 2002.

[OT4] Okonek, Ch.; Teleman, A.: Comparing virtual fundamental classes: gauge theoretical Gromov-Witten invariants for toric varieties, Asian J. Math. 7, no. 2, 167-198, 2003.

[OT5] Okonek, Ch.; Teleman, A.: Gauge theoretical equivariant GromovWitten invariants and virtual fundamental classes, math.AG/0301131, to appear in Proceedings of the Fano Conference in Torino, 2002.

[RR] Ramanan, S.; Ramanathan, A.: Some remarks on the instability flag, Tohoku Math. J. 36, 269-291, 1984.

[Ra] Ramanathan, A.: Moduli of principal bundles over algebraic curves, Proc. Indian Acad. Sci. (Math. Sci.) 65, 301-328 and 421-449, 1996.

[SU] Schoen, R.; Uhlenbeck, K.: Boundary regularity and the Dirichlet problem for harmonic maps, J. Diff. Geom. 18, 253-268, 1983.

[Sh1] Shiffman, B.: Separately meromorphic functions and separately holomorphic mappings, in "Several complex variables and complex geometry", Part 1, Proc. Santa Cruz, CA, (1989), Amer. Math. Soc., Providence, RI, 191-198, 1991.

[Sh2] Shiffman, B.: Separately meromorphic mappings into compact Kähler manifolds, Contribution to Complex Analysis and Analytic Geometry (H.Skoda and J.Trépreau eds.), Braunschweig, Germany, 243-250, 1994.

[Si] Simpson, C. T.: Higgs bundles and local systems, Inst. Hautes Études Sci. Publ. Math. No. 75, 5-95, 1992.

[Su] Suyama, Y.: The analytic moduli space of simple framed holomorphic pairs, Kyushu J. Math. Vol. 50, 65-68, 1996.

[Te1] Teleman, A. : Projectively-flat surfaces and Bogomolov's theorem on class VII $I_{0}$ surfaces, Int. J. Math. 5, 253-264, 1994.

[Te2] Teleman, A.: Non-abelian Seiberg-Witten theory and stable oriented pairs, Internat. J. Math. 8, no. 4, 507-535, 1997.

[Te2] Teleman, A. : Analytic stability, symplectic stability in non-algebraic complex geometry, preprint, math.CV/0309230, to appear in Int. J. Math.

[Th] Thaddeus, M.: Stable pairs, linear systems and the Verlinde formula, Invent. math. 117, 181-205, 1994. 
[UY1] Uhlenbeck, K.; Yau, S. T. : On the existence of Hermitian-Yang-Mills connections in stable vector bundles, Comm. Pure Appl. Math., 39, 257S293, 1986.

[UY2] Uhlenbeck, K.; Yau, S. T. : A note on our previous paper: On the existence of Hermitian-Yang-Mills connections in stable vector bundles, Comm. Pure Appl. Math. 42, 703-707, 1989.

[W] Witten, E.: Monopoles and four-manifolds, Math. Res. Letters 1, 769$796,1994$.

Authors' addresses:

Martin Lübke:

Mathematical Institute, Leiden University, P.O. Box 9512 NL, 2300 RA Leiden,

e-mail: lubke@math.leidenuniv.nl

Andrei Teleman:

CMI, Université de Provence, 39 Rue F. Joliot-Curie, F-13453 Marseille Cedex 13, e-mail: teleman@cmi.univ-mrs.fr 